\title{
The Development of a Low cost Inertial Navigation System for a Remotely Operated Vehicle
}

\author{
A thesis \\ submitted in partial fulfilment \\ of the requirements for the Degree \\ of \\ Master of Science in
}

Electronics and Computer System Engineering

at

Victoria University of Wellington

by

Jason Dean Edwards

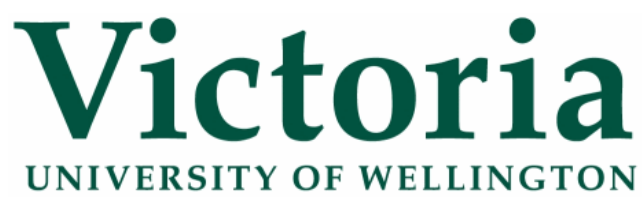

Te Whare Wānanga

o te Ūpoko o te Ika a Māui

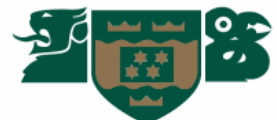


For Granddad 


\section{ABSTRACT}

Modern robotic vehicles use a large and varied set of sensors to navigate and localise their position in the environment and determine where they should be heading to accomplish their tasks. These sensors include GPS, infrared and ultrasonic range finders, laser scanners and sonar. However, the underwater environment presents challengers for modern robotic vehicles because most sensors that are typically used for navigation and localisation have reduced or no functionality underwater.

This thesis details the design and construction of a low cost Inertial Navigation System for use on the Victoria University of Wellington's (VUW) Mechatronics group Remotely Operated Vehicle (ROV). The major electronic systems, comprising of the onboard computer and microcontroller, of the ROV have been upgraded to allow for the increased computational power that the Inertial Navigation System needs and to allow further upgrading and installation of electrical and electronic systems in the vehicle as they are required. Modifications to the chassis allow quick and simple disassembly of the ROV to repair or replace major components if the need arises. 


\section{ACKNOWLEDGEMENTS}

The following people are recognised for the all the support and assistance they have provided without which this project would not have been possible.

To Dale Carnegie, my project supervisor, it has been an honour to have you as my supervisor, with your never ending enthusiasm for mechatronics and electronics and for giving me the chance to work within the Mechatronics group, which has given me new found knowledge and respect for mechatrons.

To the technical staff, Scott Forbes and Johnny McClymont, thank you for all the assistance, ideals and support you have provided. Many of the tips, tricks and suggestions you had are the sorts that can not be garnered from text books, so I thank you for teaching them to me.

To the staff in the mechanical workshop, Alan Rennie and Manu Pouajen-Blakiston, thank you for the assistance in the mechanical changes to the ROV, without which the process of accessing the ROV would have cost more time than should it have.

To my fellow graduate students, Praneel Chand, Luke Cawley, Adrian Jongenelen, Chris Lee-Johnson, Alek Ristic and David Williamson, I thank you for all the knowledge, laugher and stories shared over the length of this project. It never was a dull day in the lab, and I could never have asked for a better group of mates to study with.

To my dad, Graeme, my mum, Kathy and little brother, Steve, thank you for being there, inquiring and listening to me talk about my project even though it probably didn't make much sense. Your support throughout my university years is immeasurable.

And finally to all my friends and mates that have supported me throughout this long process I thank you. 


\section{TABLE OF CONTENTS}

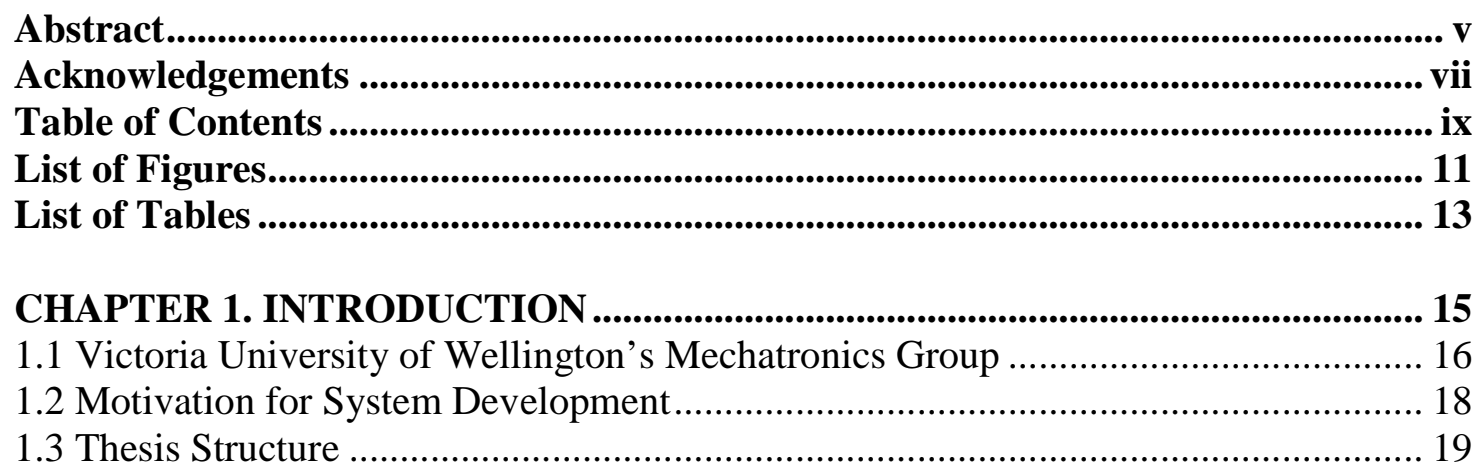

CHAPTER 2. BACKGROUND......................................................................... 20

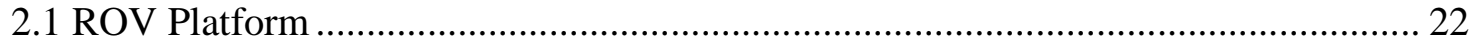

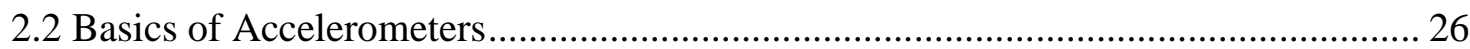

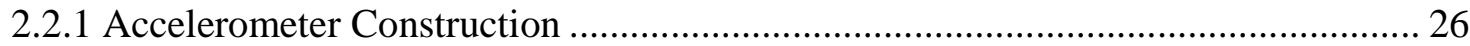

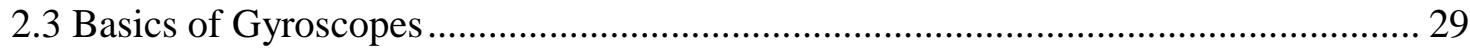

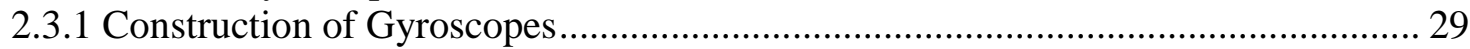

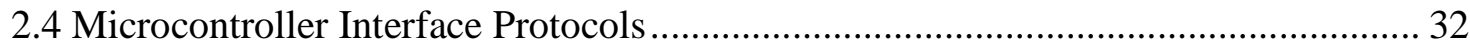

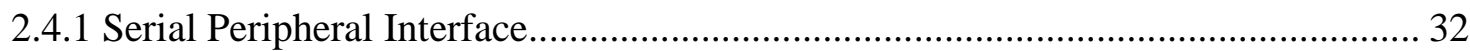

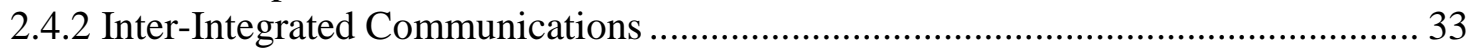

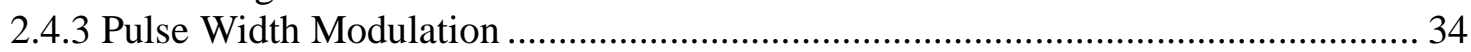

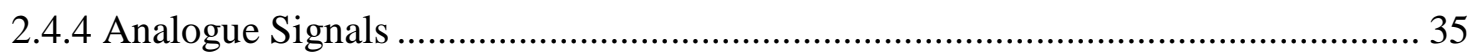

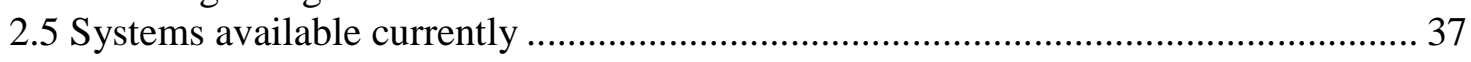

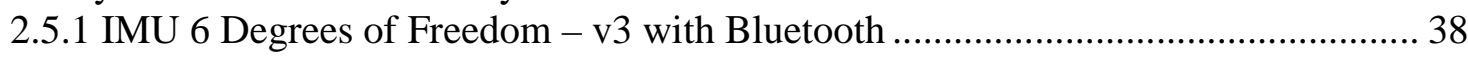

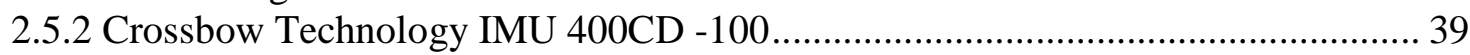

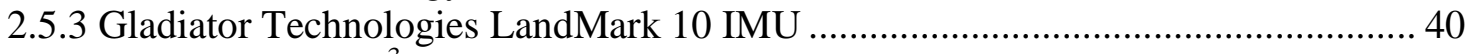

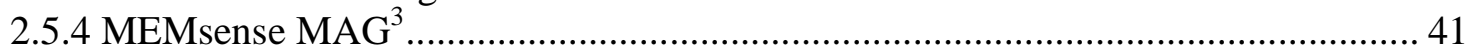

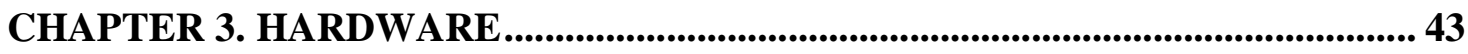

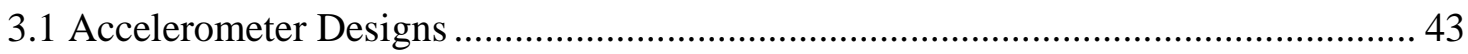

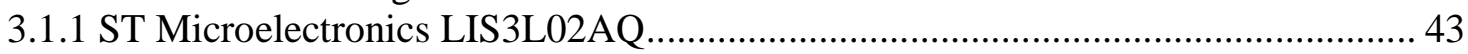

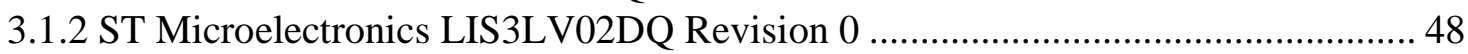

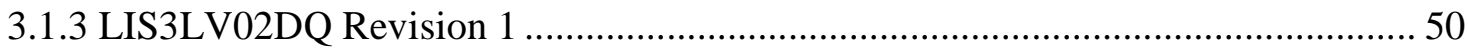

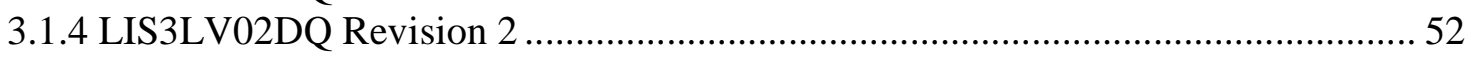

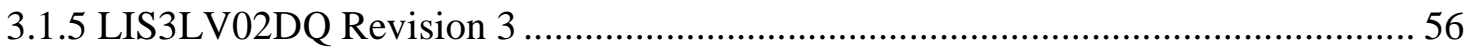

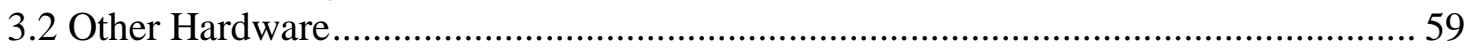

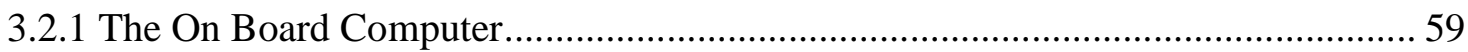

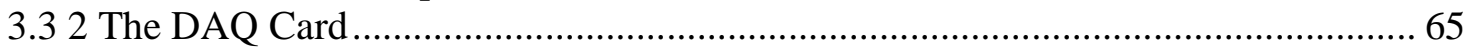

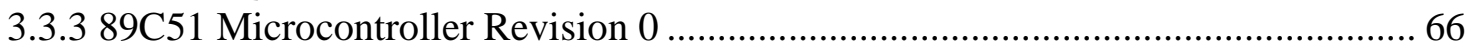

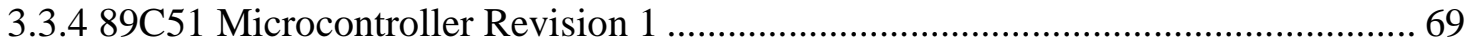




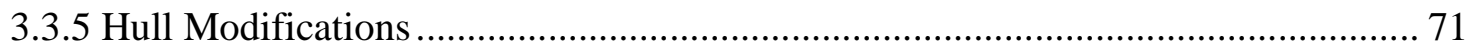

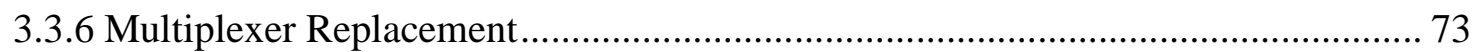

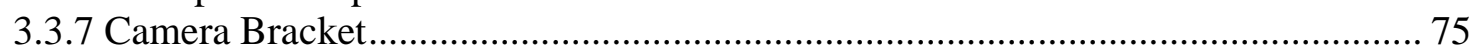

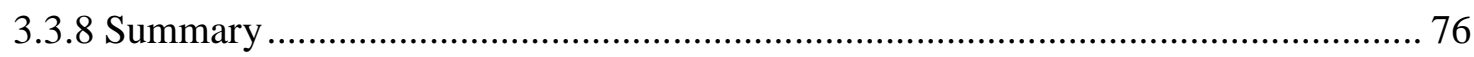

CHAPTER 4. SOFTWARE ........................................................................................... 78

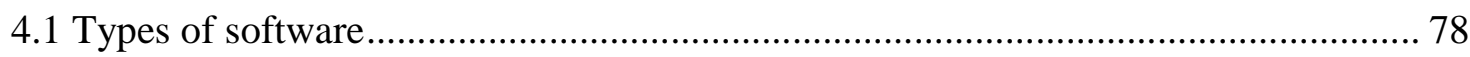

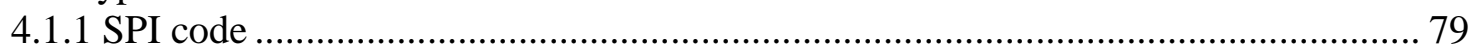

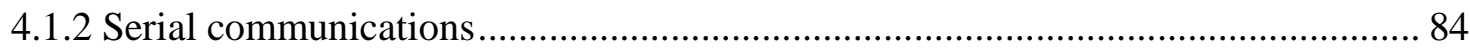

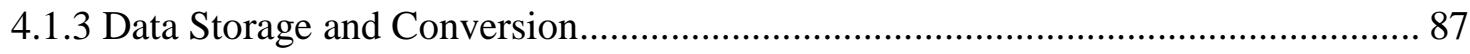

4.1.4 Mapping from One Frame of Reference to Another............................................ 94

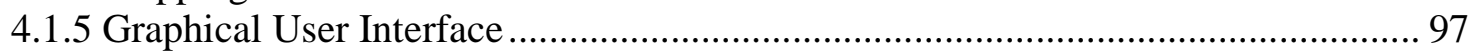

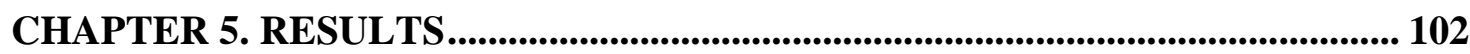

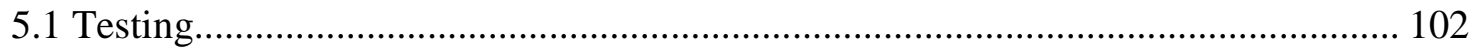

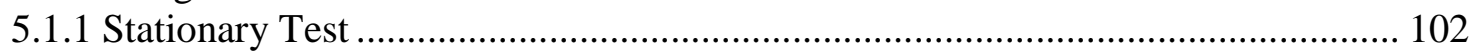

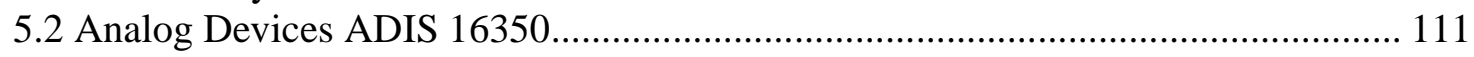

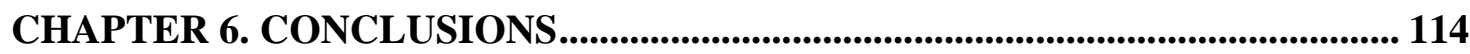

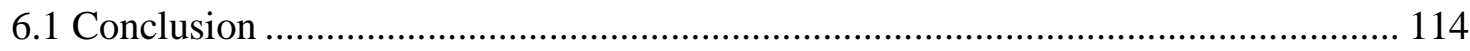

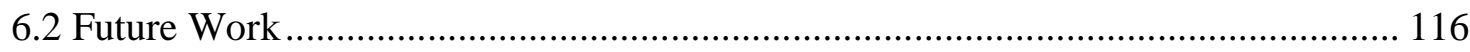

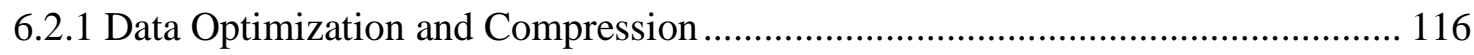

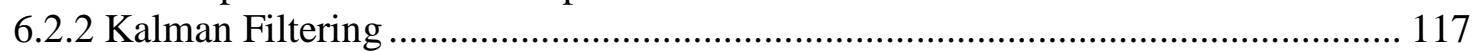

6.2.3 Solid State Hard Drive ................................................................................ 118

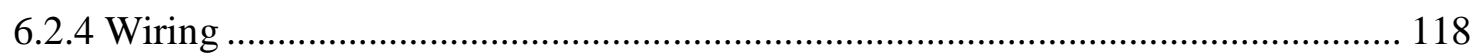

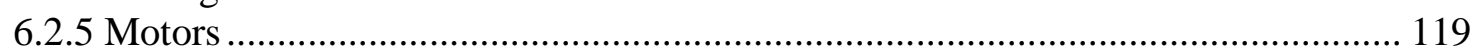

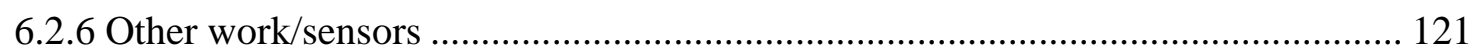

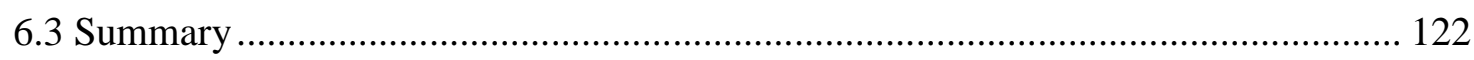

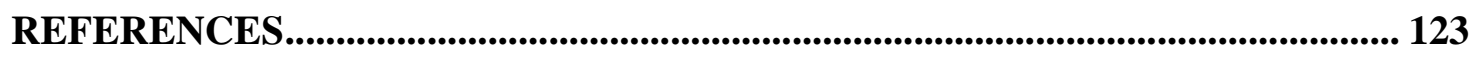

APPENDIX A: Circuit Designs ......................................................................................... 125

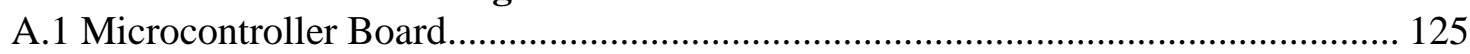

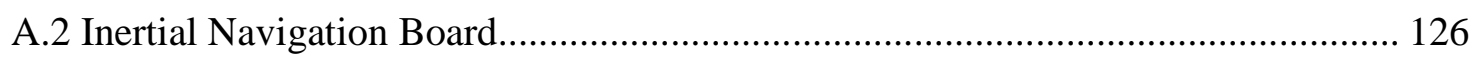

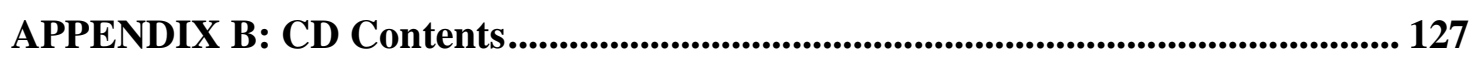




\section{List of Figures}

Figure 1.1 a) Talon Bomb Disposal Robot [1] b) Roomba Robotic Vacuum Cleaner [2] 15

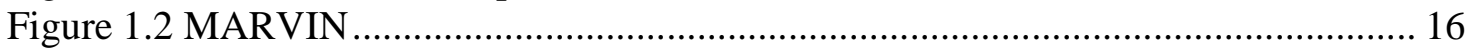

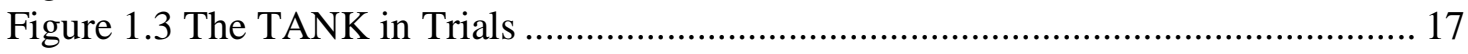

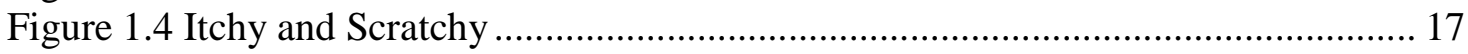

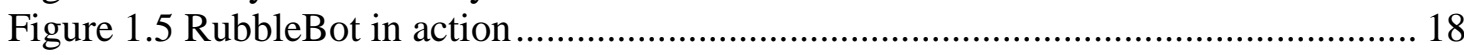

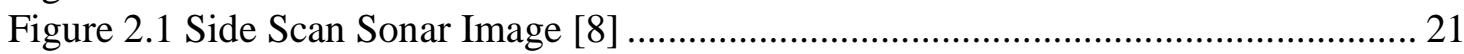

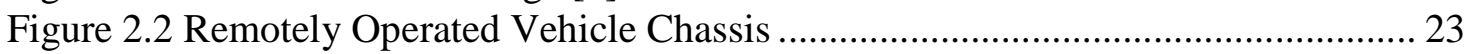

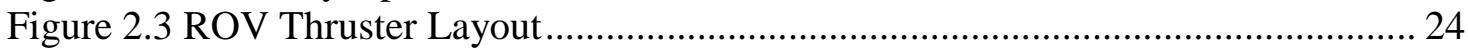

Figure 2.4 Graphical User Interface

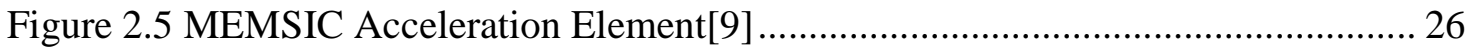

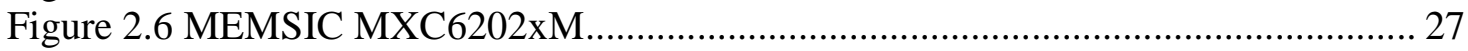

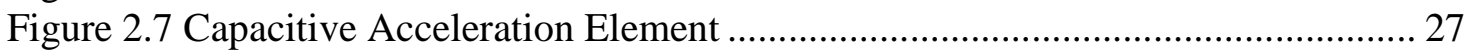

Figure 2.8 Pedestal Type Accelerometer ........................................................................ 28

Figure 2.9 iMEMS ADXRS Layout from Analog Devices ........................................... 30

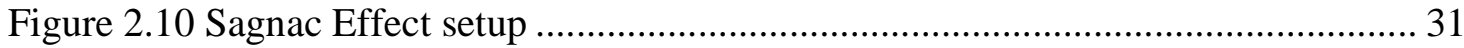

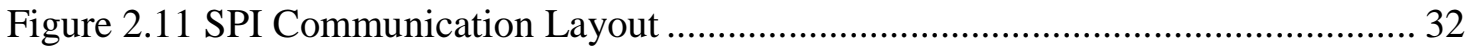

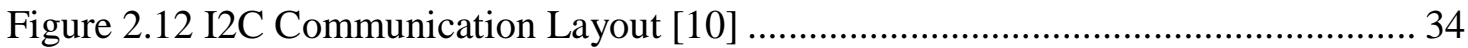

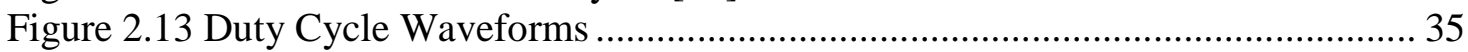

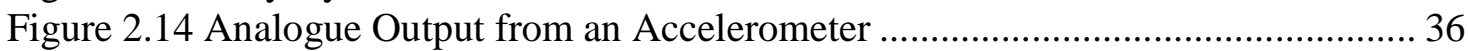

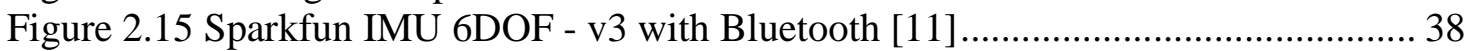

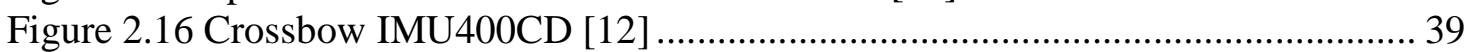

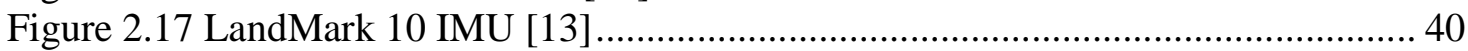

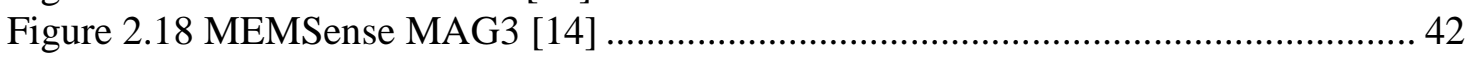

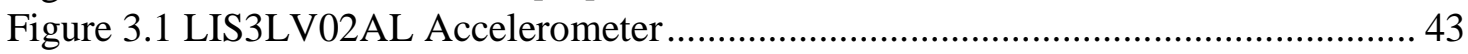

Figure 3.2 Analogue output from LIS3L02AQ3 ….................................................. 45

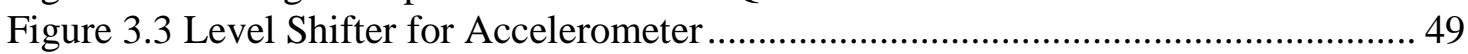

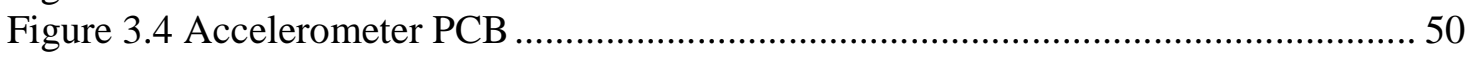

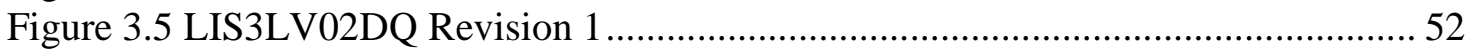

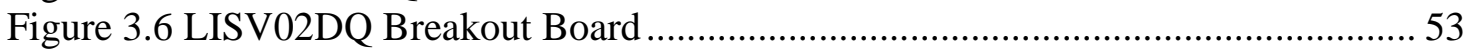

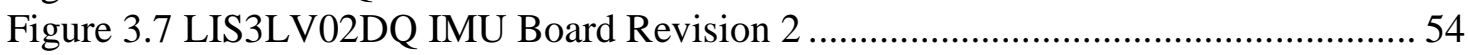

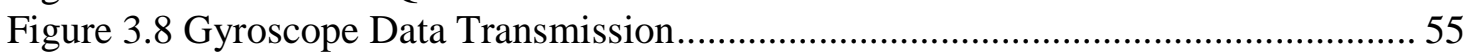

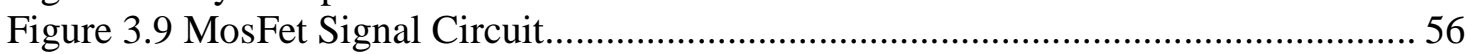

Figure 3.10 Schmitt Trigger Layout ...................................................................... 57

Figure 3.11 Mock Up Shuttle PC Motherboard Showing the Size Constraints of the Main

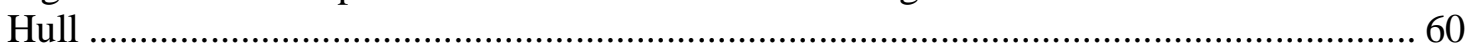

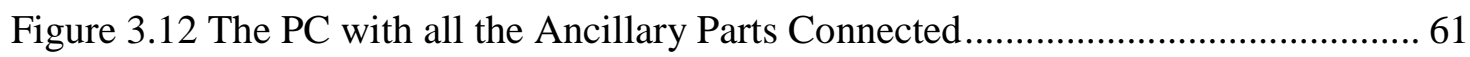

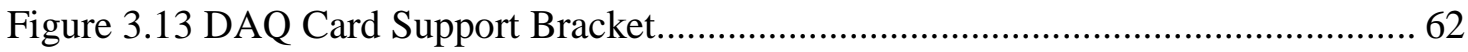

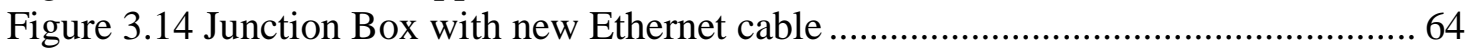

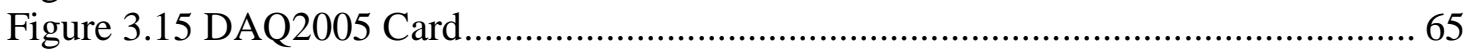

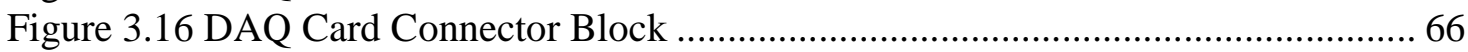




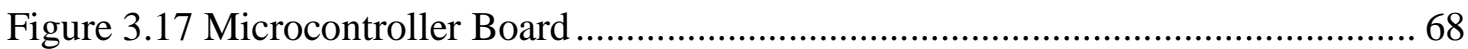

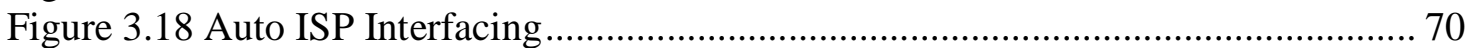

Figure 3.19 Microcontroller Board Revision 1 ............................................................. 71

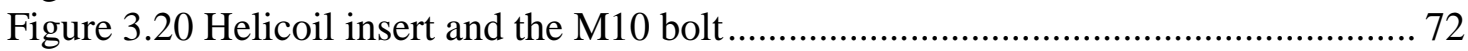

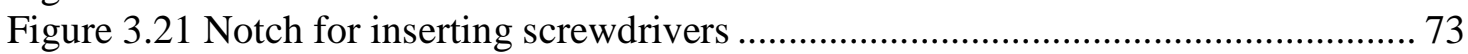

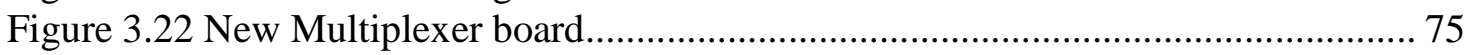

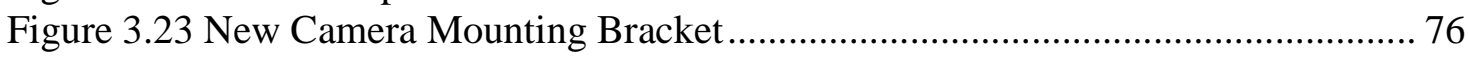

Figure 3.24 Organization Layout of the ROV and its Subsystems ................................ 77

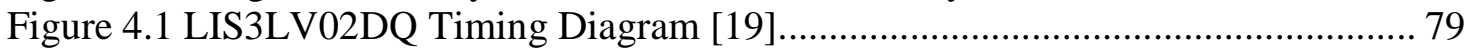

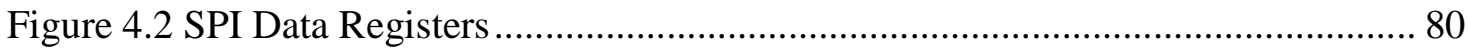

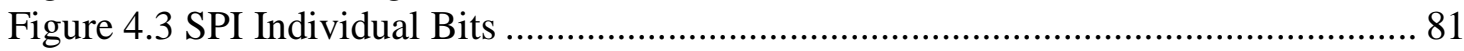

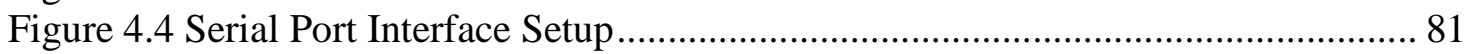

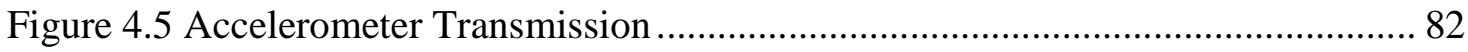

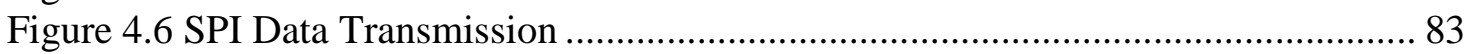

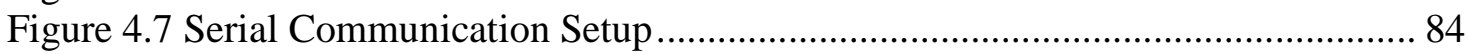

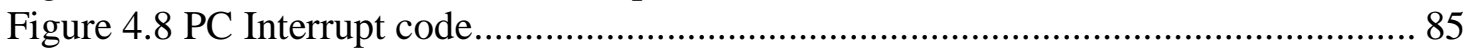

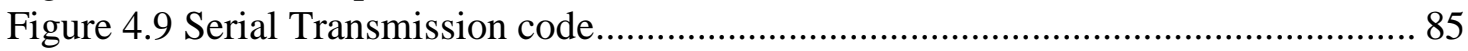

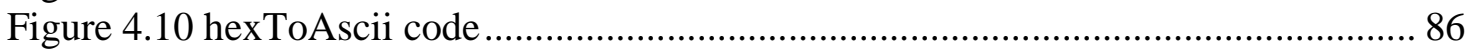

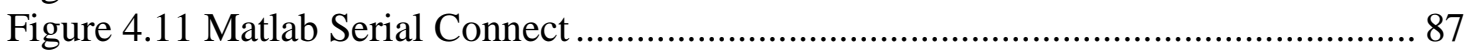

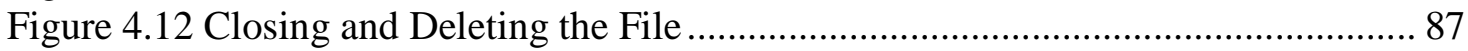

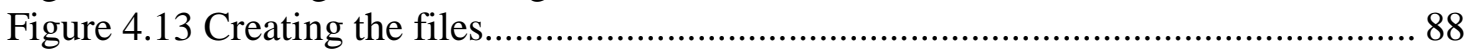

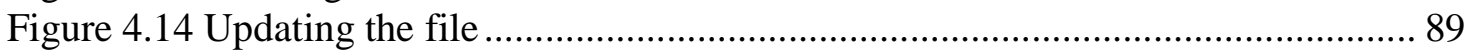

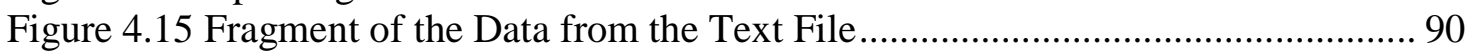

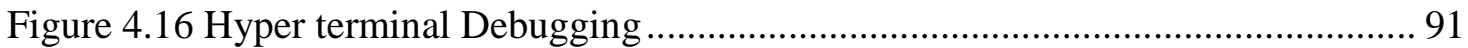

Figure 4.1716 bit (Top) and 12 bit (Bottom) data values for the $\mathrm{X}$ direction acceleration

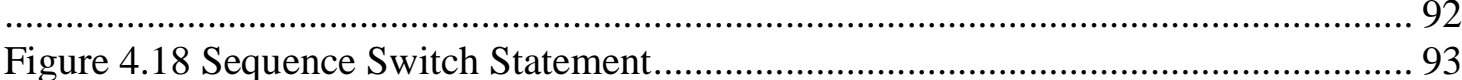

Figure 4.19 Data Conversion into Hexadecimal.......................................................... 93

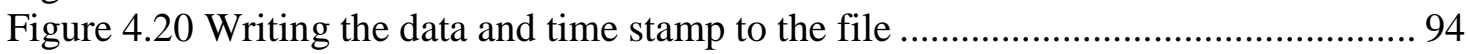

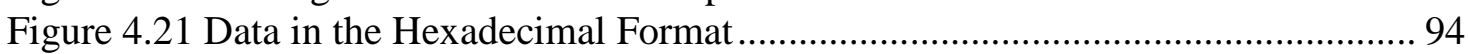

Figure 4.22 Frame of Reference Manipulation Matrices [20] .......................................... 95

Figure 4.23 Matlab Frame of Reference matrix........................................................ 95

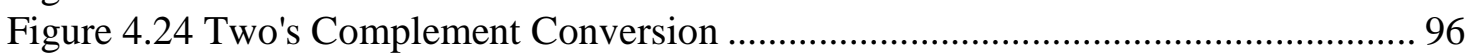

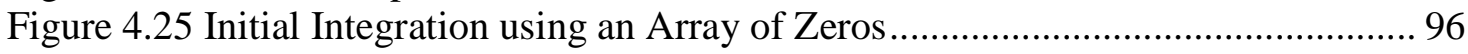

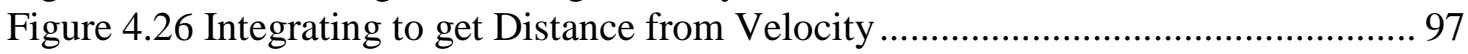

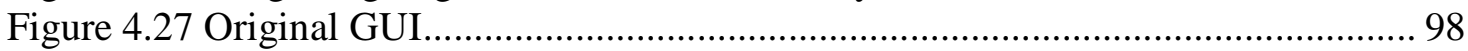

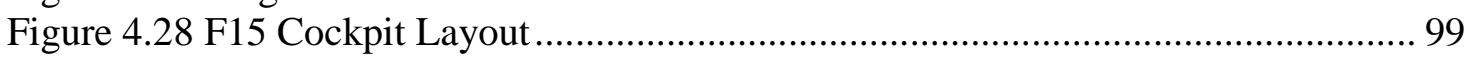

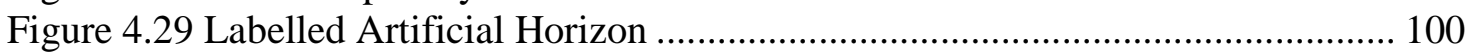

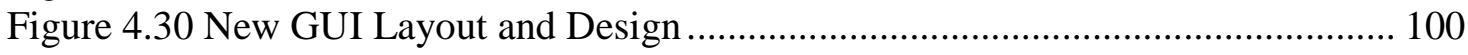

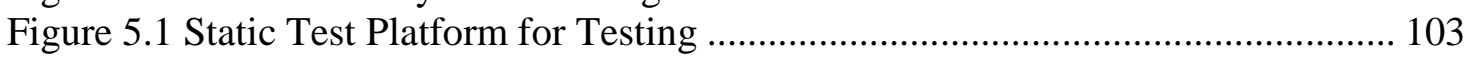

Figure 5.2 Positive X Acceleration Reading from a Static Platform ............................. 106

Figure 5.3 Negative X Acceleration Reading from a Static Platform ........................... 106

Figure 5.4 Positive Y Acceleration Reading from a Static Platform .............................. 107

Figure 5.5 Negative Y Acceleration Reading from a Static Platform ........................... 108

Figure 5.6 Positive Z Acceleration Reading from a Static Platform ............................. 108

Figure 5.7 Negative Z Acceleration Reading from a Static Platform............................ 109 
Figure 5.8 Gyroscope Reading from a Static Platform............................................... 110

Figure 5.9 Gyroscope Data Coding, Offset Binary.......................................................... 111

Figure 5.10 Analog Devices ADIS16350 Inertial Navigation Unit................................ 112

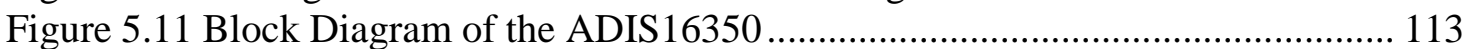

Figure 6.1 Complete Kalman filter Operation ............................................................ 117

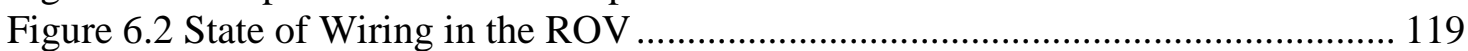




\section{List of Tables}

Table 2.1 Accelerometer Comparison Table

Table 3.1 Network Cable Comparison

Table 5.1 Example of Acceleration Data in 12 bit Right Justified Mode

Table 5.2 Example of Acceleration Data in 16 bit Left Justified Mode

Table 5.3 Median Acceleration Values

Table 6.1 Possible Motors for the ROV 


\section{CHAPTER 1. INTRODUCTION}

Mobile robots are playing an ever increasing role in the workforce. Jobs that are dangerous or hazardous and were once done by humans are now being carried out by robots (Figure 1.1). This has lead to the creation of a myriad of different robotic vehicles each with different capabilities and functions. With an increasing population that is over 65 years, mobile robots will start to be relied upon to a greater extend around the home to do everyday chores like cleaning houses, mowing lawns and assisting the elderly with tasks such as providing medication.
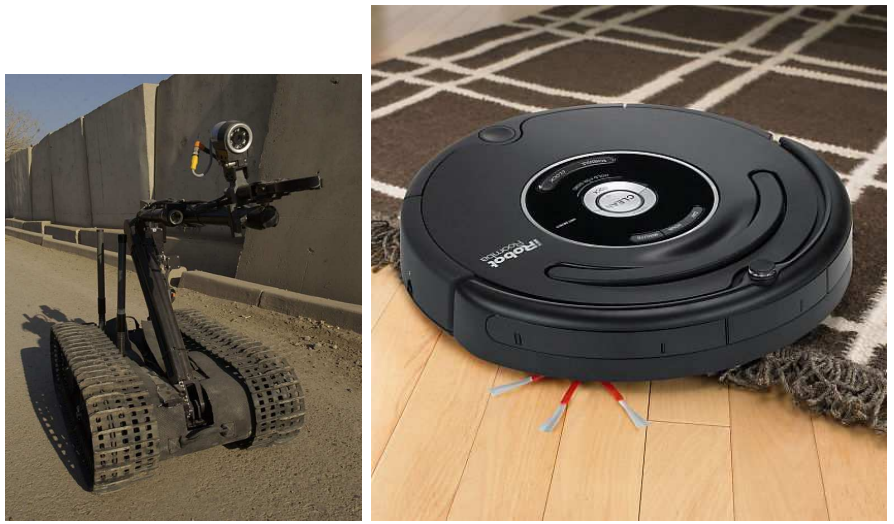

Figure 1.1 a) Talon Bomb Disposal Robot [1] b) Roomba Robotic Vacuum Cleaner [2]

All robots share a common requirement for a reliable and accurate system for navigation and localisation. Most mobile robot platforms have a large mixture of sensors to gather the data needed for the navigating the environments that they operate in. The sensors are usually a mixture of long, medium and short range sensors to provide full coverage by compensating for the blind spots that each sensor has. These data are sorted and weighted depending on how accurate the sensor is and what task the sensor is setup to measure, before being fused together to build up a picture of the environment. 


\subsection{Victoria University of Wellington's Mechatronics Group}

The Mechatronics Group has a total of six robotic vehicles to use as platforms for continuing research.

MARVIN [3] (Figure 1.2) is the oldest member of the group. He is designed as a security guard for the building, patrolling corridors and helping people if they are lost. He has a human like torso with a moveable head, neck and torso. His current sensor suite includes infrared and ultrasonic range finders and sensor processing is done on a shuttle form factor PC. He is powered by two $12 \mathrm{~V}$ car batteries and locomotion is supplied by two electric wheel chair motors in a differential drive setup.

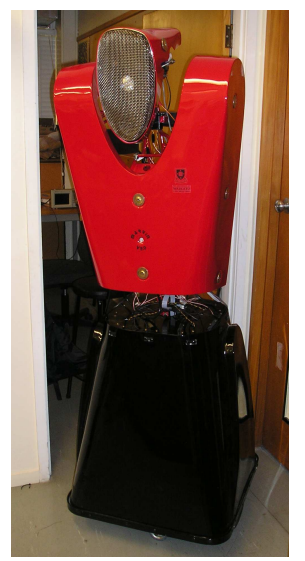

Figure 1.2 MARVIN

The TANK [4] is the second member (Figure 1.3). It is a 1.5 metre long tracked platform weighing 100 kilograms. Just like MARVIN, it is powered by two $12 \mathrm{~V}$ lead acid car batteries and has electric wheel chair motors in a differential drive setup. A shuttle form factor PC handles and processors all the data received from the compass, infrared, GPS and accelerometer sensors. Its main tasks include agricultural, horticultural and forestry applications. 


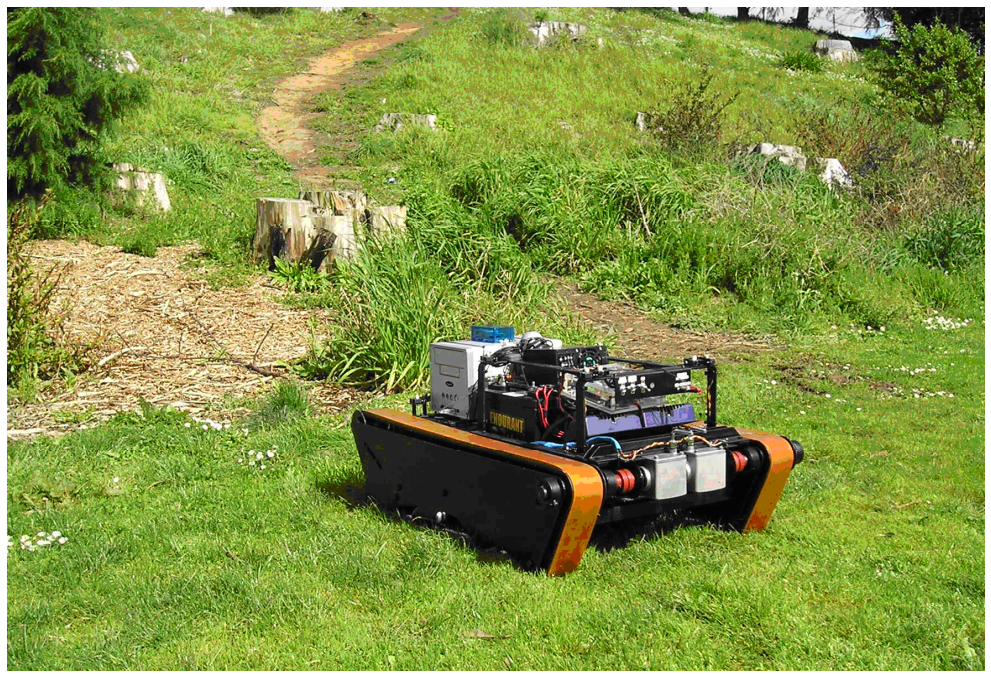

Figure 1.3 The TANK in Trials

ITCHY and SCRATCHY [5] are the Group's co-operating vehicles are shown in Figure 1.4. They are designed to work together to accomplish a task. The motivation is that instead of building larger robots to do certain tasks such as picking up steel beams, you get many smaller robots to work together to lift the beam. These vehicles have a tricycle configuration with the single wheel at the back providing the steering while the two main wheels are chain driven. Two $12 \mathrm{~V}$ lead acid batteries again power the vehicle and a micro ATX size PC handles all the processing. Sensors include infrared range finders, shaft encoders and GPS.

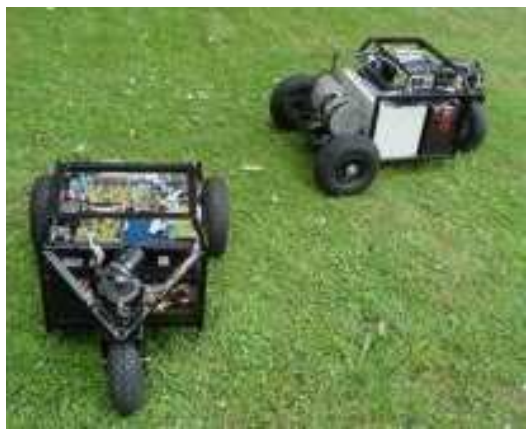

Figure 1.4 Itchy and Scratchy

RUBBLEBOT [6]is the newest addition to the group (Figure 1.5). It is an urban research and rescue vehicle that traverses a damaged environment releasing "Daughters" which are miniature robots that penetrate into the rubble. The Daughters report if they have found something that may be a human to the Mothers who then send a wireless message 
to the Grandmother which acts as the base station and is responsible for directing the human rescue workers. The "Mother" Rubblebot will carry 20 to 30 of these Daughters and drop them off as it travels around the area. The Rubblebot has a Nano based PC with a AMD Geode $800 \mathrm{CPU}$ on it for its processing as well as an ARM9 embedded controller. It is powered by $4 \mathrm{Li}$-ion batteries giving it an endurance of up to 3 hours.

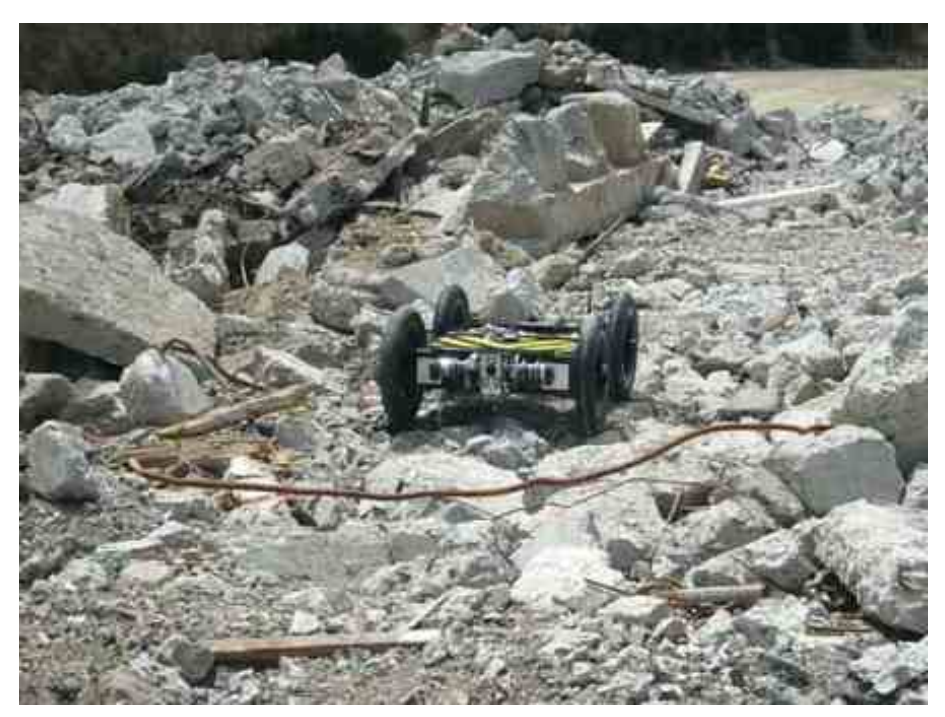

Figure 1.5 RubbleBot in action

Lastly is the ROV [7]. Section 2.1 will go into more detail on the ROV, but its applications are underwater surveying and exploration.

\subsection{Motivation for System Development}

The ROV is Victoria University of Wellington's underwater submersible vehicle. It is a two part system made up of a surface based part which is made up of the operator's PC and the transformer box which provides power to the sub-surface vehicle below the water, via an umbilical cable that connects the surface and sub surface parts together.

There is a need for a system that shows the operator the current position of the robot underwater without having a diver swim alongside the vehicle. Ideally the system created for the ROV could also be used on the other robots in the Mechatronics Group to increase 
the sensor suites of the vehicles, aiding in localisation and facilitating the improved creation of maps that the vehicle's make as they travel.

\subsection{Thesis Structure}

The thesis structure is laid out:

Chapter 2 Outlines the types of sensors currently available for navigation purposes, the current platform for which the sensor system will be mounted, and some of the commercially available systems.

Chapter 3 Describes the design and implementation of all new hardware components for the platform.

Chapter 4 Details the software created or updated to allow communication between the hardware and the operator's controls.

Chapter 5 Details the procedure and results obtained from testing the sensor under several scenarios.

Chapter 6 Briefly outlines the implications of the thesis and details possible future work. 


\section{CHAPTER 2. BACKGROUND}

A major obstacle with mobile autonomous robots today is how to determine their precise location. For a mobile robot to determine its location, the robot has to know a) where it is now and b) where it is going. Knowing where the robot is going is especially important in places where the robot has no landmarks to reference itself against.

There are many different ways to determine a mechatrons current position and direction. Modern sensors include Global Positioning Systems (GPS) which is used to find the absolute position, Light Detection and Ranging (LIDAR), Sonar, Infrared sensors and cameras for object or feature recognition, and Inertial Navigation Systems (INS), shaft encoders to find the relative position. Most modern mechatrons use a combination of these sensors, compensating somewhat for the limitations of each.

While land and aerial based vehicles can use most (if not all) of these systems, underwater vehicles are limited by the water medium. GPS cannot function under water because the frequency of its waves cannot propagate through water. Object recognition fails because there are generally very few fixed position objects under water for the robot to compare against. LIDAR works only to a certain depth, and its performance is dependent on the clarity and the thermal layers of the water.

Sonar is a distance measurement techniques based on ultrasonic sensors and is commonly used in a navigation tool in the marine environment. It works by sending out a high frequency sound wave that is reflected or redirected from obstacles or the sea floor. These waves are then detected by an array of microphones and are digitally reconstructed to provide a 3D map of the area that has been scanned or 'pinged'. 


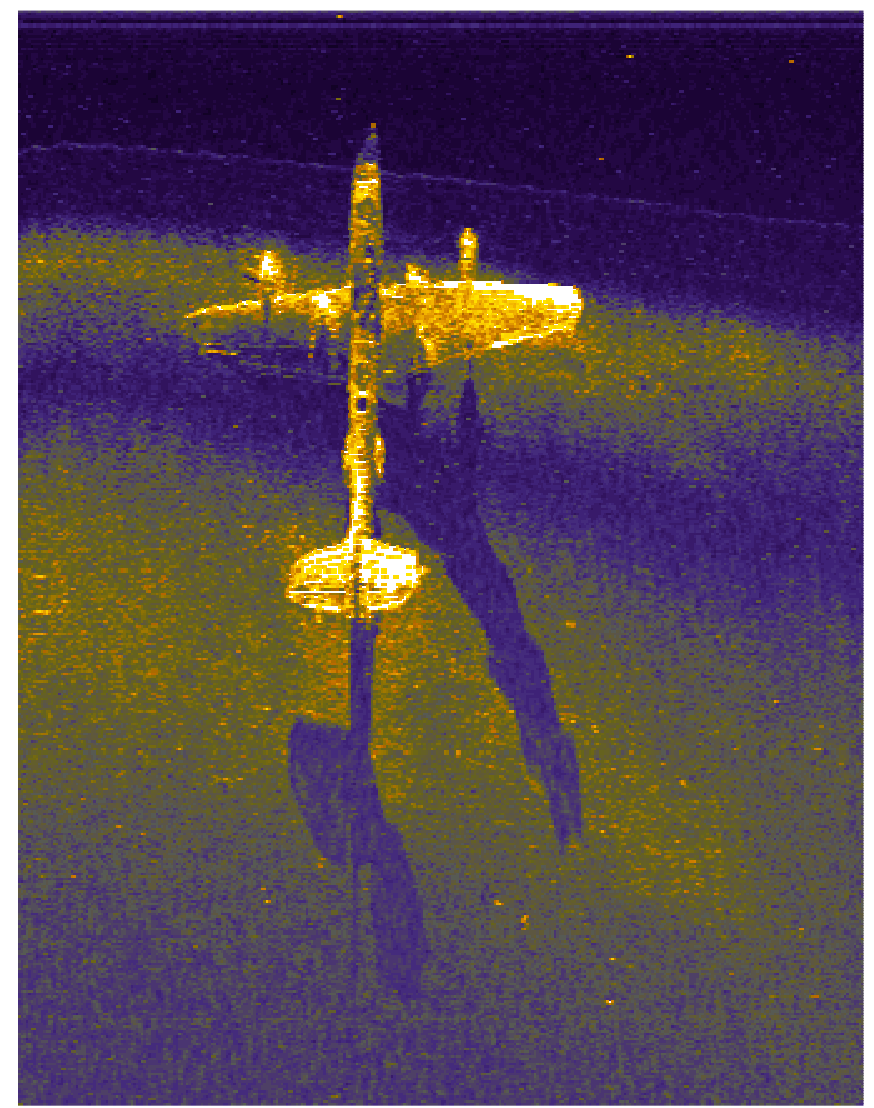

Figure 2.1 Side Scan Sonar Image [8]

The types of sonar systems available depend on the task the vehicle is doing. If the vehicle is surveying the sea floor then a side scan sonar or a multiple beam system would be used to scan large areas simultaneously (Figure 2.1). Fish finders are a commercially available system based on sonar, used by fishers to find fish and the sea floor. They have a narrow field of view and cannot be used while the vehicle is travelling at some speeds as motor noise corrupts the signal.

Another system commonly employed on underwater vehicles is the Inertial Navigation System (INS). INS does not rely on external signals to determine its position. It has been heavily favoured by the military for some years because it does not send out or receive signals and consequently is immune to jamming. For many years INS used mechanical gyroscopes to determine the position of the vehicle. More recently however, ring laser gyroscopes, vibrating gyroscopes and accelerometers and magnetometers have been implemented [9]. 
INS measures the vehicle's acceleration and rate of angle change, and then converts these data into a velocity and current position. The current position is based on the distance from the start position, which must be independently determined. To settle any disturbances in the INS when it is first powered up, it is common practice to have the vehicle stationary for about five minutes before moving it in order to create a reference point.

It is with the latest technologies of nanoscale production, Very Large Scale Integration (VSLI), that some of the biggest movements in INS have occurred. Increasing miniaturization of electronic devices has made it possible to manufacture large numbers of devices with high accuracy and low foot print size, which means that the overall size of the INS has been dramatically reduced. This has helped the devices become more common in everyday life, such as the accelerometer in some laptops and hard drives to protect them if dropped, and gyroscopes and accelerometers in cars (they stabilise larger vehicles by helping to prevent roll overs and activating airbags in accidents).

It was decided to implement an INS because the vehicle may be in operation some distance from the bottom of the body of water, which would limit the effectiveness of sonar.

\subsection{ROV Platform}

The ROV comprises an aluminium chassis with a wooden floatation block on top and measures $1200 \mathrm{~mm} \times 500 \mathrm{~mm} \times 460 \mathrm{~mm}(\mathrm{~L} \times \mathrm{W} \times \mathrm{H})$. This block gives the vehicle a slightly positive buoyancy factor, which allows the vehicle to be recovered in the case of a loss of power. 


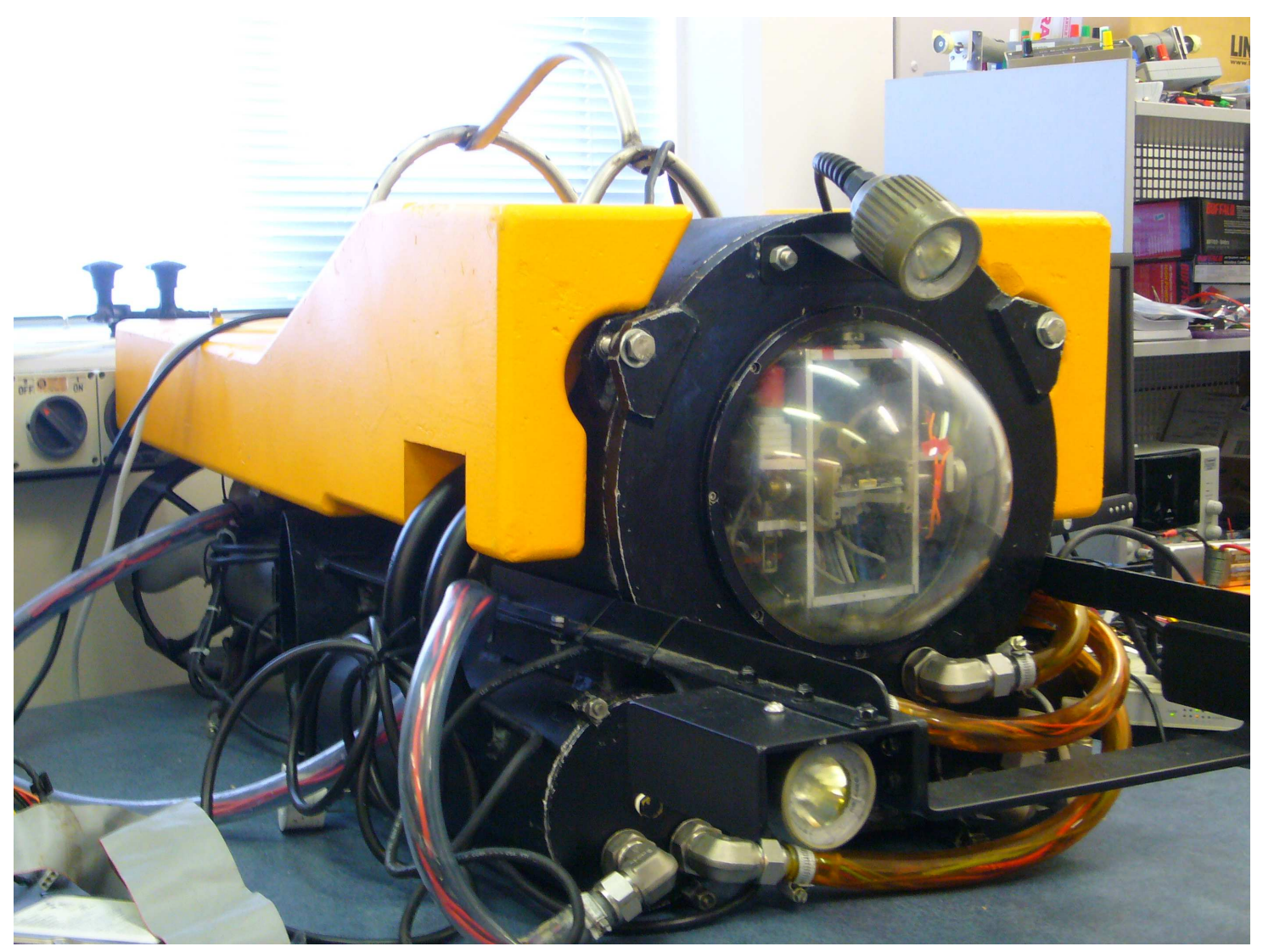

Figure 2.2 Remotely Operated Vehicle Chassis

The thruster power to control the ROV is obtained from four AC powered industrial drill motors configured as in Figure 2.3, with two motors facing rearwards on the port and starboard side, a lateral mounted motor to skid the vehicle left and right, and a vertical mounted motor to submerge it. 


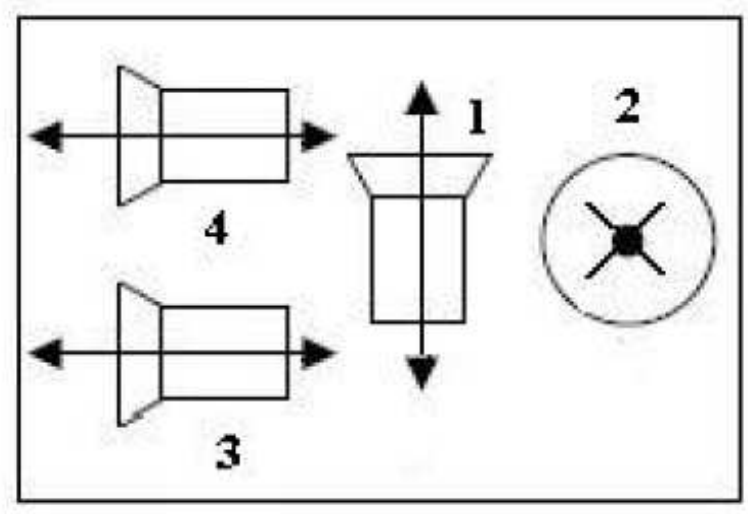

Figure 2.3 ROV Thruster Layout

A 240 VAC transformer provides the power for the vehicle from either the shore or a launch vehicle. This power is transmitted to the ROV through the umbilical tether attached to the chassis. The tether contains three sets of power cables - one each for the motors, the ROV's computer and the sensor electronics. Also included in the tether is the twisted pair network cable, which is connected to the operator's laptop PC from which the ROV is controlled.

The sensors currently installed include a pressure sensor, a compass, a tilt and roll sensor, humidity, temperature and a leak detection sensor. All of these data are displayed on the operator's PC, which has a Graphical User Interface (GUI) for the robot (Figure 2.4). A joystick can also be connected to control the ROV.

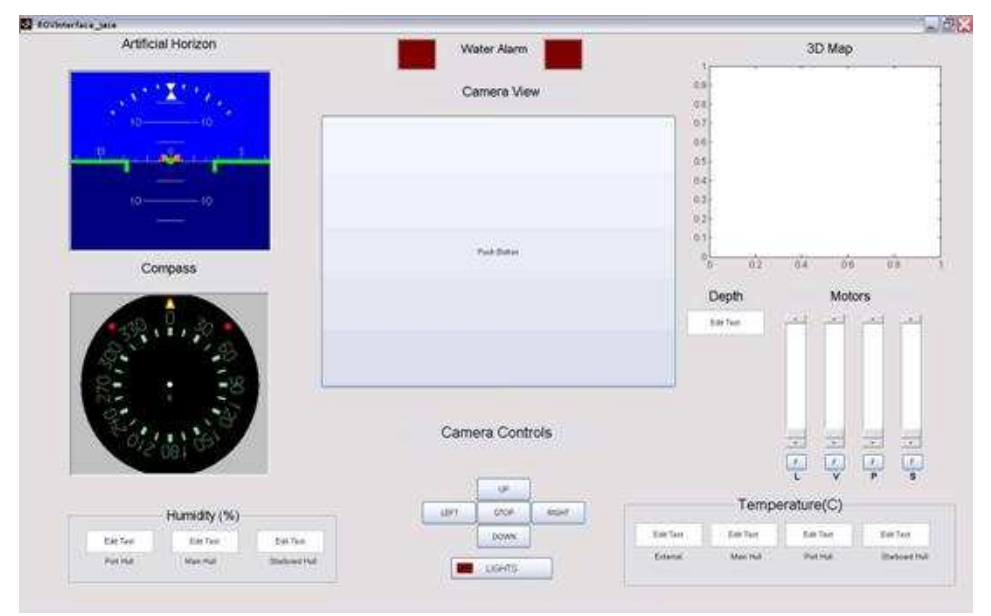

Figure 2.4 Graphical User Interface 


\subsection{Basics of Accelerometers}

\subsubsection{Accelerometer Construction}

Accelerometers are constructed to measure acceleration in one of two ways. Each manufacturer adds their own intellectual proprietary rights to the construction and internal interface of the devices, such as microcontrollers or signal processors and analogue to digital converters [10].

The first method is to use a heating element in the centre of a sensor array (Figure 2.5). This creates a group of heated particles that are detected by the temperature sensor arrays in the $\mathrm{X}, \mathrm{Y}$ and $\mathrm{Z}$ direction. As the sensor is accelerated the heat travels in the opposite direction to the acceleration and the arrays pick this up.

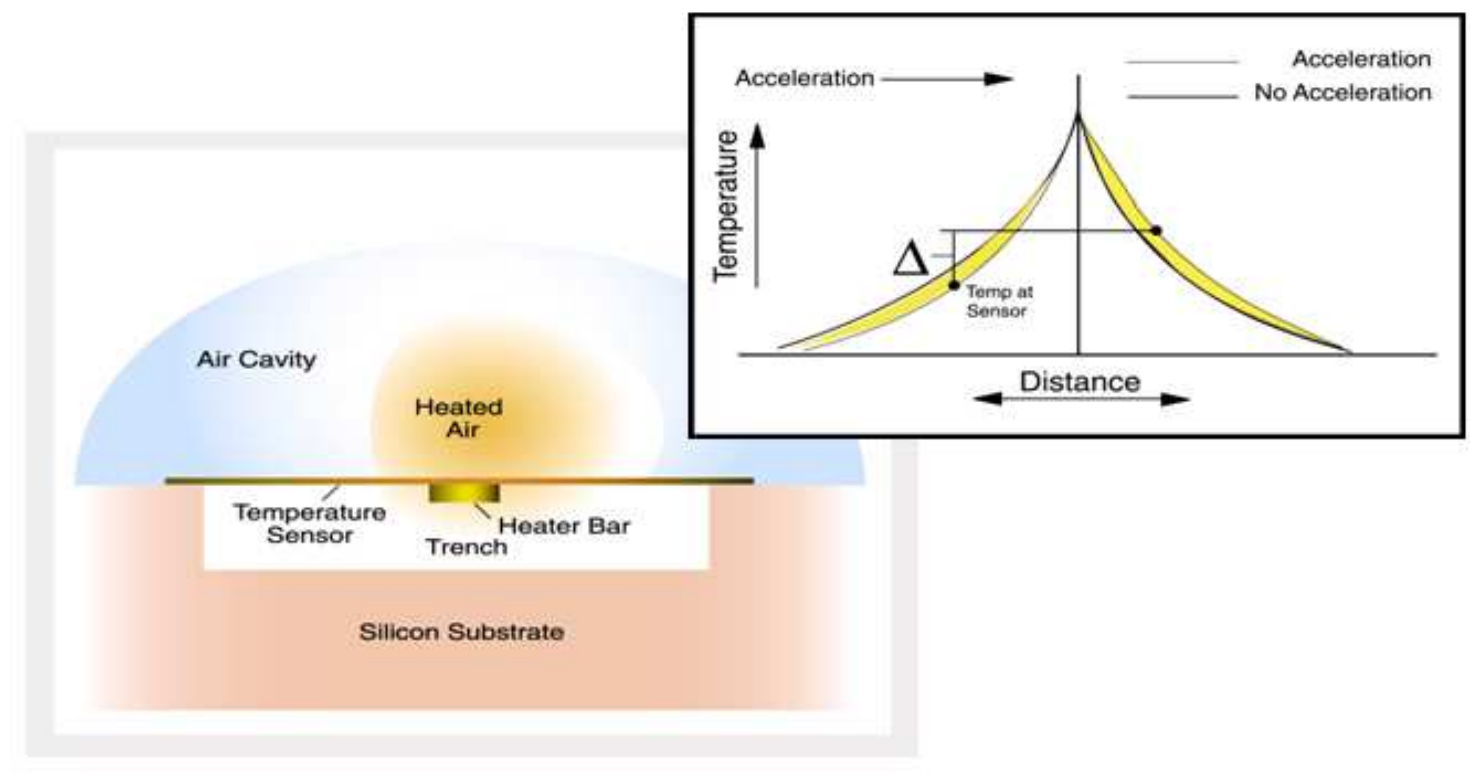

Figure 2.5 MEMSIC Acceleration Element[11]

The signal from the arrays is fed into the conditioning part of the chip, which converts the signal to a form that can be passed to external devices. This type of acceleration sensor can be seen below in the MEMSIC MXC6202xM accelerometer. 


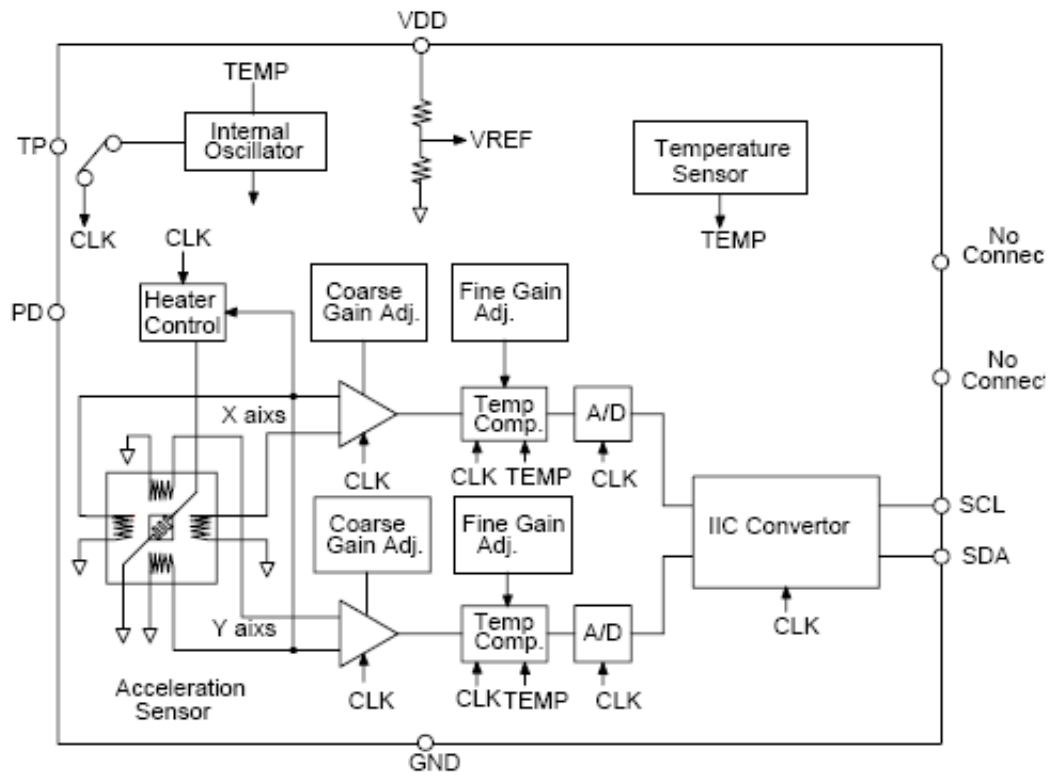

MXC6202G/H/M/N FUNCTIONAL BLOCK DIAGRAM

Figure 2.6 MEMSIC MXC6202xM

From the functional diagram Figure 2.6 it can be seen that there is also an internal temperature sensor. This is to compensate for a bias that occurs in most devices if used at a temperature other than that specified in the datasheet of the chip. Typical values for bias are between $0.005 \%$ and $0.5 \%$ per degree of temperature. In this accelerometer the communication interface to a microcontroller is by the $\mathrm{I} 2 \mathrm{C}$ protocol.

The second way to measure acceleration is to create a capacitive pair of plates with a movable middle plate. The movable plate initially sits equidistantly between the two outer plates.

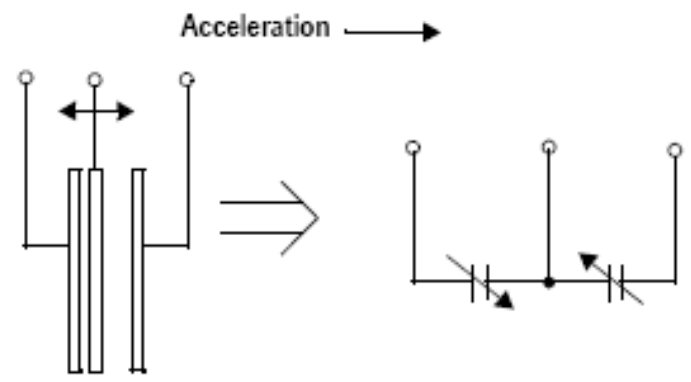

Figure 2.7 Capacitive Acceleration Element 
When acceleration occurs, the middle plate moves in the opposite direction of the applied acceleration. This changes the capacitance of the capacitive pair: one capacitor increasing in capacitance and one decreasing.

A slight variation on this method is to have a central pedestal on which the top plates rotate. One of the capacitor plates is embedded in the substrate, so that when acceleration is detected the top plate will spin in the direction of acceleration. Whether an increase or decrease in capacitance occurs depends on the position of the plates.

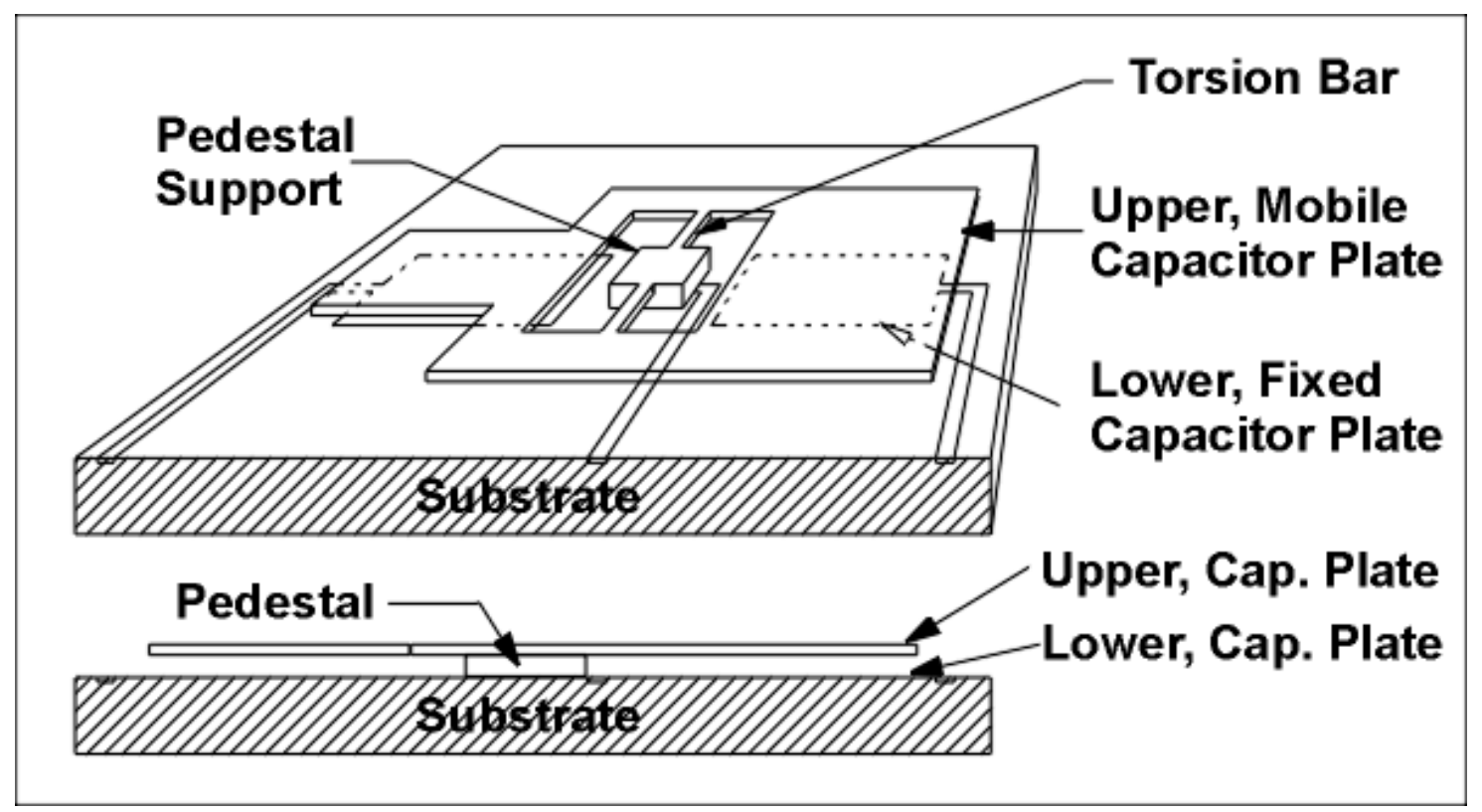

Figure 2.8 Pedestal Type Accelerometer

The measured capacitance is then passed to a sample and hold circuit where it is converted into either an analogue signal, which is directly outputted, or into a digital signal which is stored into the memory or registers of a microcontroller. The most common methods for transferring the signal to the microcontroller are Serial Peripheral Interface (SPI), Inter-Integrated Circuit (I2C), Pulse Width Modulation (PWM) and by measuring the pure analogue signals. 


\subsection{Basics of Gyroscopes}

\subsubsection{Construction of Gyroscopes}

Gyroscopes have been in use since the turn of the twentieth century. Early models were based on a spinning plate that resisted or measured the change in orientation of the spinning plate.

Modern gyroscopes still use a spinning wheel in some applications. However, with the advent of solid state technology and miniaturisation, gyroscopes can be based on resonators, vibrating structures, or interference patterns in light [10].

All gyroscopes are based on measuring the Coriolis effect. This effect is calculated using the formula:

$$
F=2 M v \times \Omega
$$

$F$ denotes the Force, $v$ is the velocity of the mass $M$, and $\Omega$ is the angular velocity of the rotating frame of reference with $\times$ being the cross product.

Resonating or Vibrating Ring gyroscopes measure the Coriolis effect by driving a frame to resonance. This frame will keep vibrating if the supporting frame it is attached to is rotated. A transducer detects the responding Coriolis effect, and the signal is (usually) fed through some gain and sampling circuits before being transmitted off the chip. The communication interfaces are exactly the same as those used for accelerometers: mainly SPI, I2C, PWM and analogue signals. Figure 2.9 shows the internal layout of an Analog devices gyroscope. 


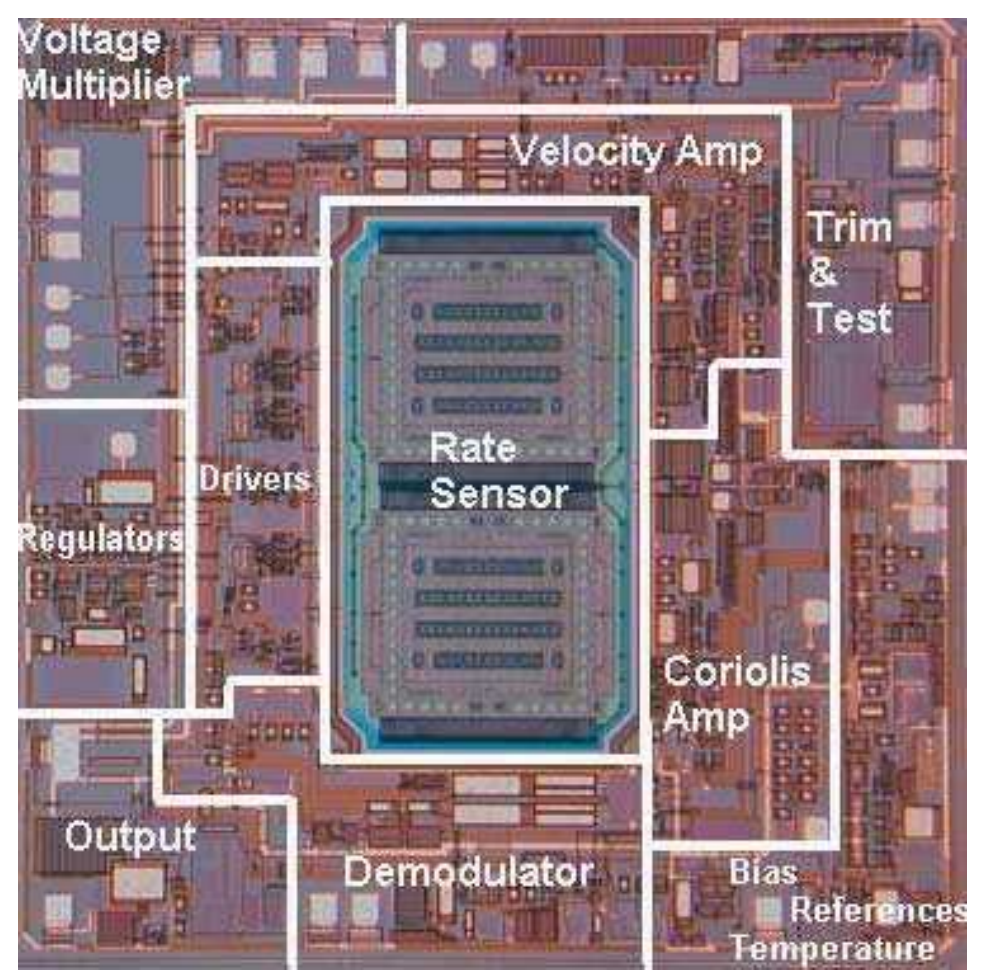

Figure 2.9 iMEMS ADXRS Layout from Analog Devices

As mentioned, another way to measure the Coriolis effect is using interference patterns in light. This is known as the Sagnac effect, after its discoverer George Sagnac. He showed that if a beam of light is split into two parts and sent around a ring in opposite directions, when they meet up again there will be a fringe pattern (Figure 2.10). This fringe pattern occurs because one of the beams will be longer than the other, depending on the direction the detector was rotating at the time. These interference patterns can be created with lasers and mirrors (Ring Laser Gyros)[12] or using light fed into fibre optic cables (Fibre Optic Gyros)[13]. 


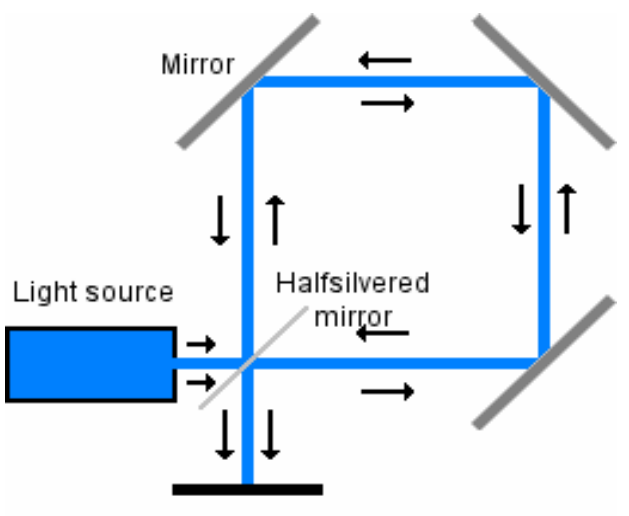

Viewing screen

Figure 2.10 Sagnac Effect setup 


\subsection{Microcontroller Interface Protocols}

\subsubsection{Serial Peripheral Interface}

SPI involves a minimum of four lines, which are the Serial Clock (SCLK), the Serial Data Output or Master Output Slave Input (SDO or MOSI), the Serial Data Input or Master Input Slave Output (SDI or MISO) and the Slave Select (SS) line, to control a number of different devices (Figure 2.11).

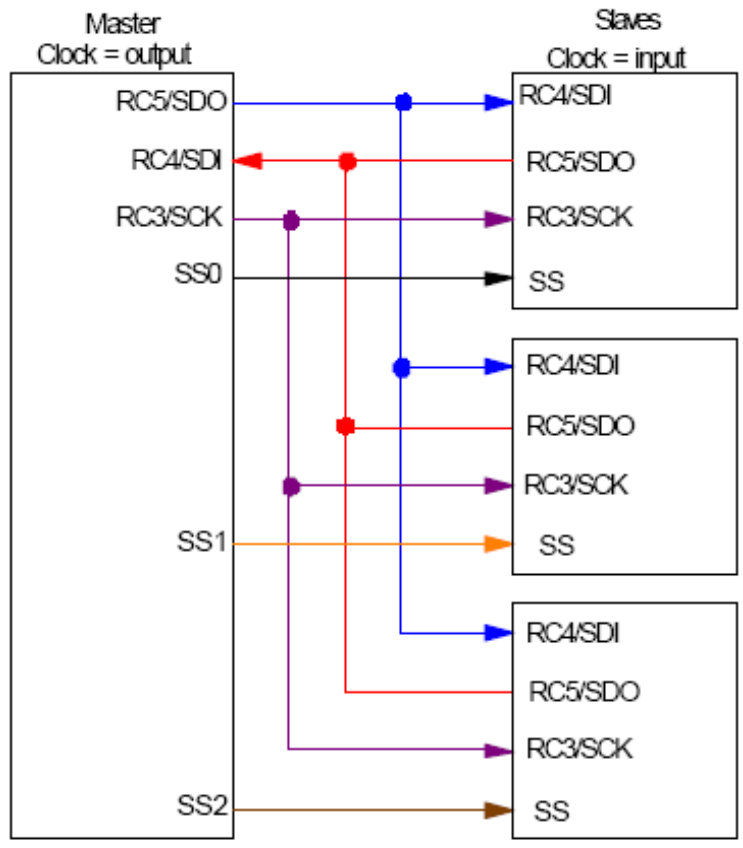

Figure 2.11 SPI Communication Layout [14]

The hierarchy of these devices is a master and slave one. The master initiates the contact with the slave by setting the Slave Select (SS) line of the particular device to low. Each slave usually has a separate SS line. If there is only one slave then this line is usually tied low. All the other lines are shared with each slave device.

Once a slave receives a signal on its slave line it prepares to accept a request from the master. This is sent down the SDO line from the master and is clocked into the slave via 
the SCK line. When the slave is ready to transmit back to the master it waits for the appropriate timing on the clock line then sends the data request back to the master on the SDI line.

The way this system of communication is set up means it is possible to have slave to slave and master to master communication. This means that a few of these devices can be set up to form a small network.

SPI is full duplex, meaning that information can be sent and received at the same time, so it can be used for high speed communication and can reach data rates up to 10Mbits/s.

\subsubsection{Inter-Integrated Communications}

I2C is an older communication protocol developed in the 1980s by Philips to communicate between low speed peripherals using two wires: a Serial Data (SDA) line and a Serial Clock (SCL) line.

The bus master is the first device to signal that it intends to transmit data on the bus. It sends out a signal on the SDA line and every other device attached to the line becomes active listening. The bus master then transmits the address of the device the data will be going to.

Every device on the I2C bus has a unique address. When the address is transmitted on the SDA line all devices on the line check it with their own. If they match then the device waits for the data to be sent to it. If the address does not match the device steps back from the line and waits until another signal tells the devices to listen.

Because I2C only has two lines, one control and one data, it can only communicate at half duplex speed. This means it cannot send and receive data at the same time. The latest version of $\mathrm{I} 2 \mathrm{C}$ has a typical rate of $400 \mathrm{Kbits} / \mathrm{s}$ in fast mode and up to $3.4 \mathrm{M}$ bits/s in high speed mode. 


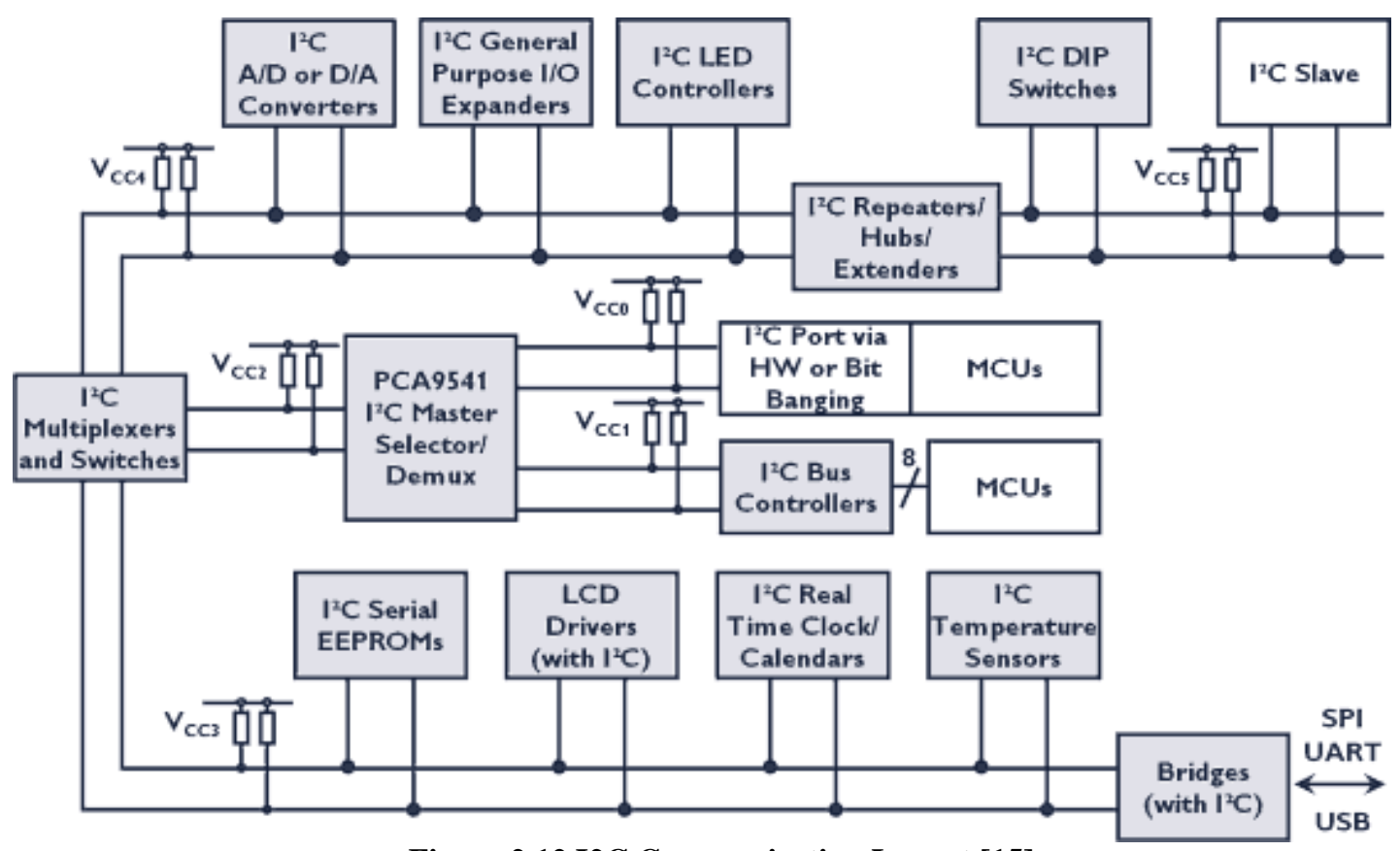

Figure 2.12 I2C Communication Layout [15]

I2C is still commonly used and as shown in Figure 2.12, there are many different types of devices that work on the protocol. The more devices that are plugged into the $\mathrm{I} 2 \mathrm{C}$ bus, the more pull up resistors are needed to keep the voltage on the lines high, which is the idle state for the $\mathrm{I} 2 \mathrm{C}$ communication.

\subsubsection{Pulse Width Modulation}

Pulse Width Modulation (PWM) is another popular communication protocol that accelerometers use to communicate with external devices. PWM is a waveform that has a varying duty cycle: this determines the length of time the pulse is on or off. A $50 \%$ duty cycle means that the waveform has a binary value of 1 for $50 \%$ of the time and a binary value of 0 for the remaining $50 \%$ of the time. For example if an accelerometer is sensing no acceleration the duty cycle would be $50 \%$, whereas if a positive acceleration was detected the duty cycle would increase above $50 \%$ (based on how much force the accelerometer detected). 


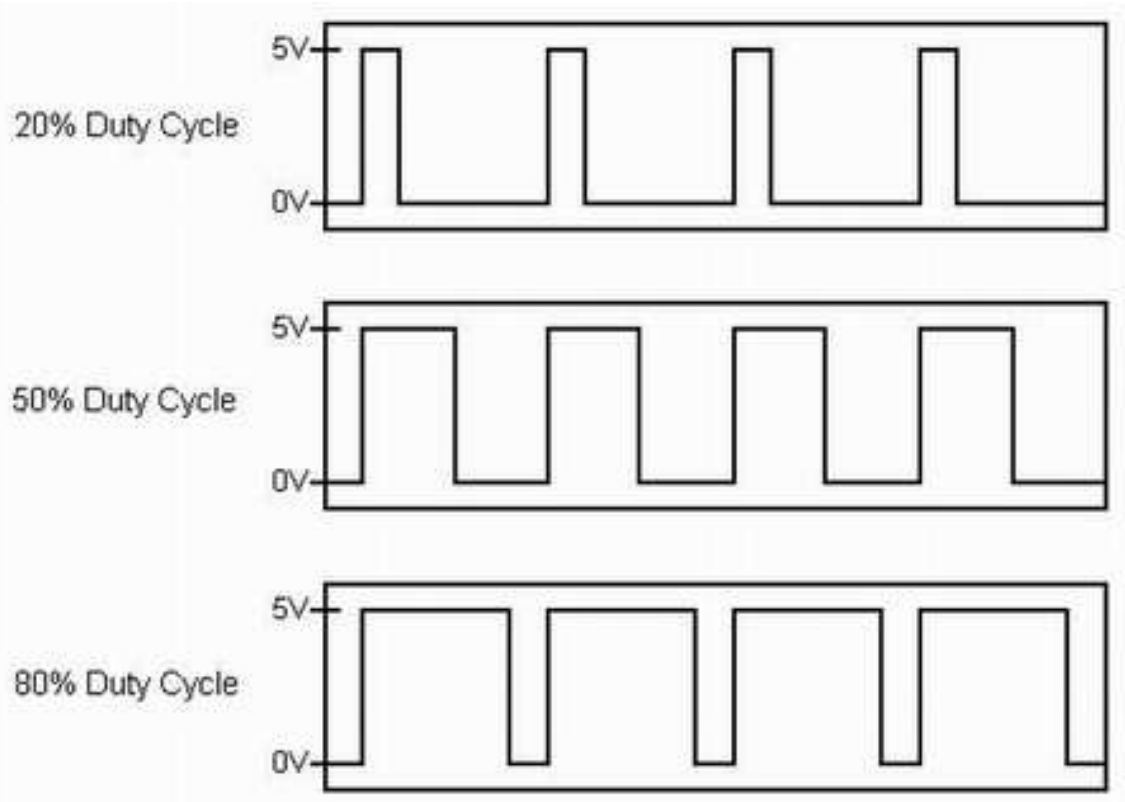

Figure 2.13 Duty Cycle Waveforms

The Figures above represent the output from a $+/-2 \mathrm{~g}$ accelerometer: a $20 \%$ duty cycle would represent a $-1.2 \mathrm{~g}$ acceleration while a $80 \%$ duty cycle would represent a $+1.2 \mathrm{~g}$ acceleration.

The PWM signal can be passed straight into a microcontroller to display or act upon the signal. PWM is commonly used to control motors, dimmers in light switches, and other devices where accurate control is required. PWM tends to be highly energy efficient, because the signal is made up of pulses that are usually high frequencies and are switching between high and low (depending on the duty cycle) very quickly

\subsubsection{Analogue Signals}

Accelerometers can output an analogue signal. An analogue signal has to be converted into a form that can be easily understood by the microcontroller: this is the task of an Analogue to Digital converter (ADC). This can be a stand-alone chip or as with some microcontrollers, can be built into the microcontroller. 


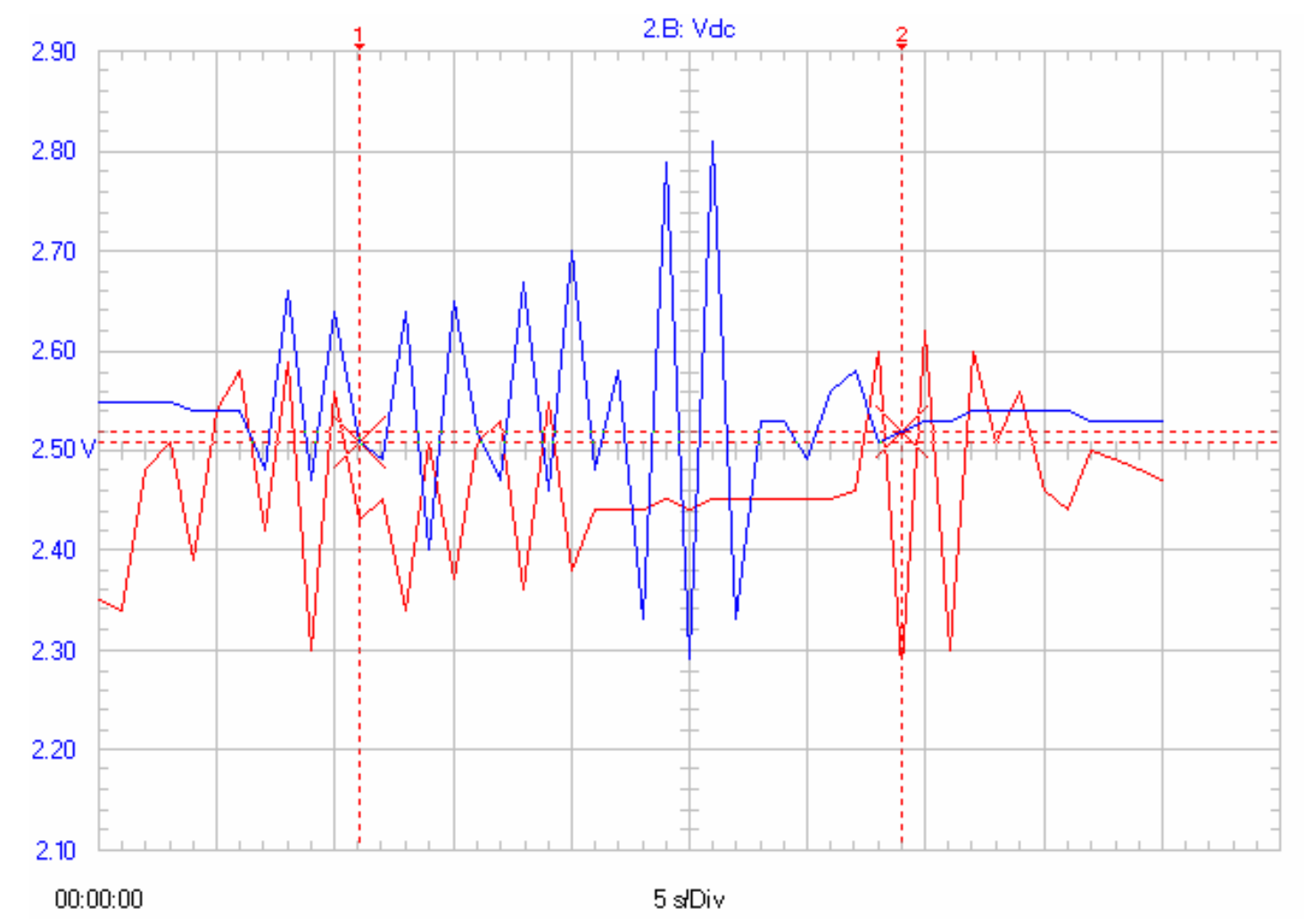

Figure 2.14 Analogue Output from an Accelerometer

The potential precision of an ADC depends on the number of bits used to represent the analog quantity. Usually the representation is 10 or 12 bits but it is possible to get 14 and even 16 bit ADCs. When using 14 or 16 bit ADCs the signal has to be extremely clear because any noise on the transmission line will drown out the precision of the extra 2 or 4 bits. Figure 2.14 shows an analogue 10 bit signal from an analogue accelerometer which was initially tested for this project. The two lines, blue and red represent a separate channel in the accelerometer.

The major problem with analogue signals is that there is a high likelihood of noise on the transmission lines if the source of the signal is some distance away from the ADC and/or runs near high voltage components or a source of high magnetic fields (such as motors)[17]. These can be filtered out, although filtering increases the complexity of the circuit and can also remove some of the data at the lower end bits. 


\subsection{Systems available currently}

As soon as the ROV leaves visual range of the surface vessel or of known underwater features it is essentially 'flying' in unknown territory. There is currently no way for the operator of the ROV to know anything more than which way the vessel is pointing or its depth.

To position the ROV underwater in relation to the operator's frame of reference using an INS, the current position of the ROV in the vehicle's own frame of reference must be found first. This has to take into account not only the distance travelled by the vehicle but also its orientation.

The orientation and distance of a vehicle in all three directions gives a 6 Degree of Freedom range of motion. Any system that is to be employed on the ROV must have this as a minimum. Since there are few known obstacles underwater to take distance measurements from (using the likes of IR or laser sensors), accelerometers are used to calculate the distance.

The density of the medium through which the ROV will be travelling sets the specifications for the sensors and systems that are to be studied. A maximum of $+/-2 \mathrm{~g}$ $\left(19.6133 \mathrm{~ms}^{-2}\right)$ in acceleration is set because the maximum acceleration of the ROV in operation, including a current, is unlikely to be greater than this. The maximum rate of roll in the three directions is set at 80 degrees per second. If the conditions are above those specified then it is highly likely that the weather conditions would be too rough to launch the vehicle in the first place.

The interface of the sensor system will most likely be a digital signal such as RS232, RS45 or other similar communication protocol. Nevertheless, analogue systems will still be considered since the option to connect them into the DAQ card's analogue port is available if required. 


\subsubsection{IMU 6 Degrees of Freedom - v3 with Bluetooth}

This INS unit comprises a single Freescale MMA7260Q 3 axis accelerometer, two Invensense IDG300 dual axis gyroscopes and a Honeywell HMC1043 dual axis magnetometer with a Bluetooth transmitter.

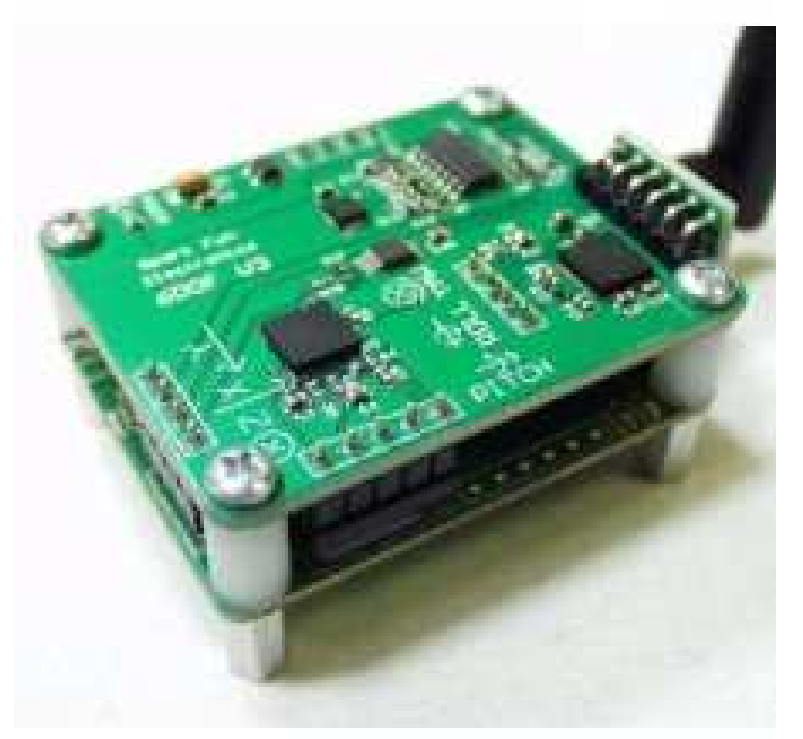

Figure 2.15 Sparkfun IMU 6DOF - v3 with Bluetooth [17]

The board's specifications are:

○ Selectable g sensitivity of $1.5 \mathrm{~g}, 2 \mathrm{~g}, 4 \mathrm{~g}$ or $6 \mathrm{~g}$

○ Gyroscope rate change of 300 degree/second

○ Full scale field range $+/-6$ gauss

○ LPC2138 family microcontroller

The Freescale MMA72600 is a capacitive type accelerometer as mentioned in Section 2.2.1, while the Invensense IDG300 gyroscope is a vibrating mass (Section 2.3.1) gyroscope made with the company's proprietary construction techniques for gyroscopes. 
This product is aimed both at robotic hobbyists, and companies that want a prototype of a system so that it can be evaluated for use in their products or for research into their own designs. The boards come preassembled, so once they are connected to a power source and the program is downloaded via a computer the INS can be left running.

Because of the close proximity of the INS and the PC in the vehicle, the use of a straight cable connection is the best communication setup for the ROV. While the Bluetooth communication will mean fewer cables have to be placed in the vehicle, a Bluetooth kit would also have to be purchased for the PC to enable this interface.

Price was also considered, and at US\$449.95 from Spark fun Electronics (not including the Bluetooth connection kit required for the ROV) the 6 DOF IMU was considered to be outside the project's budget. The unit that is to be used in the ROV is to be low cost while having similar if not better specifications than this device to ensure that it is better suited for the task.

\subsubsection{Crossbow Technology IMU 400CD -100}

The Crossbow IMU400CC is a 6 Degree of Freedom solid state inertial package.

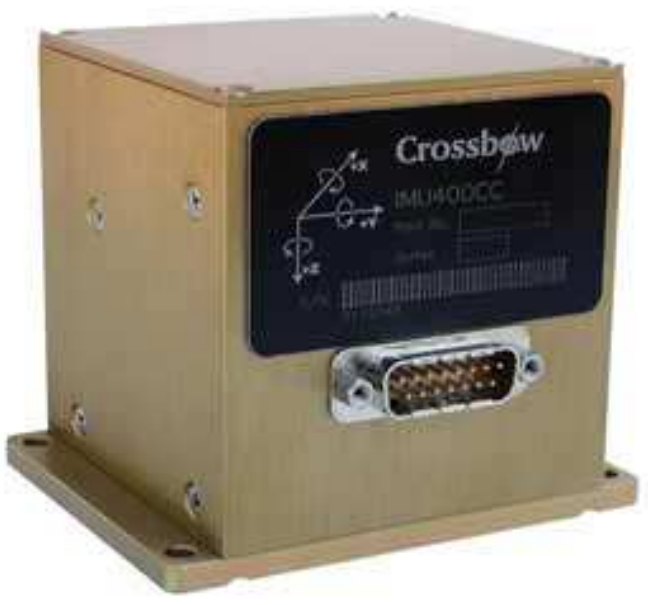

Figure 2.16 Crossbow IMU400CD [18] 
Although the manufacturer does not list the accelerometer or gyroscopic components that are incorporated in this device, it does list that the accelerometer is an analogue type triaxial accelerometer that may be susceptible to noise from any power lines or signals that run close to the interface. There is also a digital output in a RS-232 format. The specifications for both the -100 and -200 models respectively are:

- Angular rate of 100 or 200 degrees per second

○ Acceleration range of $+/-2 \mathrm{~g}$ or $+/-10 \mathrm{~g}$

○ 14 bit A/D converter

This device already has a protective cover (see Figure 2.15), which is an aluminium case with mounting points already on it. This device was not chosen for several reasons. The first is size: the dimensions of the aluminium case are $93.5 \mathrm{~mm} \times 76.2 \mathrm{~mm} \times 83 \mathrm{~mm}(\mathrm{~L} \times$ $\mathrm{W} \times \mathrm{H})$, so it would take up too much space in the vehicle's limited hull (160 mm deep $\times$ $350 \mathrm{~mm}$ diameter, which also contains the existing electronics).

The second reason it was not chosen was the price. The IMU400CC-100 is available from Mandeno Electronic Equipment Ltd for NZ\$9495.02 including GST which is not within the project's budget range.

\subsubsection{Gladiator Technologies LandMark 10 IMU}

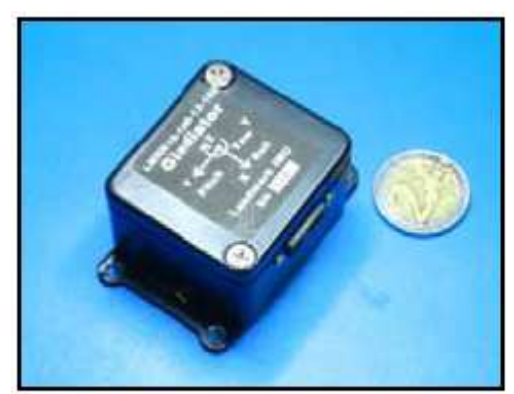

Figure 2.17 LandMark 10 IMU [19] 
The specifications for this IMU are:

○ +/- 75, +/- 150, +/- 300 degrees per second

$\circ+/-1.7,+/-12 \mathrm{~g}$ acceleration

○ 3 Internal Temperature Sensors

- Digital Processor with built in A/D converter

This IMU is an all-silicon-based device with 6 degrees of freedom. It is a low powered device consuming only $1 / 3$ of a Watt in typical operation, with a mass of under 122 grams, in a small package less than $67.5 \mathrm{~cm}^{3}$. It interfaces with a PC via a RS485 connector.

While this device is perfect in terms of size and power for the ROV's purpose, the cost of the device (US\$3,800) was again outside the budget of the project.

\subsubsection{MEMsense $\mathrm{MAG}^{3}$}

Touted as the world's smallest analogue inertial measurement unit, the $\mathrm{MAG}^{3}$, measuring only $18 \mathrm{~mm} \times 18 \mathrm{~mm} \times 10.23 \mathrm{~mm}(\mathrm{~L} \times \mathrm{W} \times \mathrm{H})$, contains everything needed for selfcontained INS. It has a tri-axial gyroscope, a tri-axial accelerometer and a tri-axial magnetometer and comes in 6 different ranges:

$\circ+/-2 \mathrm{~g}$ or $+/-5 \mathrm{~g}$ acceleration range

$\circ+/-150,+/-300$ or $+/-1200$ degrees per second rate changes

$\circ+/-1.9$ Gauss magnetic field strength 


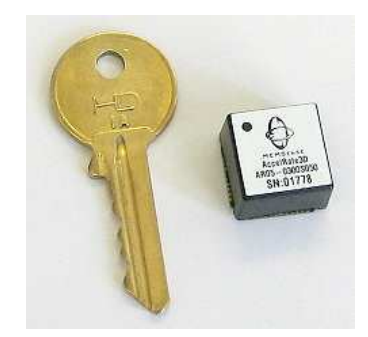

Figure 2.18 MEMSense MAG3 [20]

This is also an analogue output device with a temperature compensation circuit built in to limit the offset errors.

Although a very compact solution to an INS, the price of AUD\$1905.00 from Omni Instruments is again outside the budget of the project.

\section{Outcome}

These units cover a broad range of the commercially available products that can be purchased from international suppliers. They comprise of a mix of digital and analogue devices, in mostly small sizes and all designed for use in robotic navigation or INS.

After investigating the range of currently available products it was decided to create an IMU specific to the ROV's needs. This IMU will be an accelerometer-based design to reduce its size so that it is better accommodated into the ROV's hull, and also to reduce the cost to under US\$500. 


\section{CHAPTER 3. HARDWARE}

\subsection{Accelerometer Designs}

\subsubsection{ST Microelectronics LIS3L02AQ}

The initial accelerometer used for testing purposes was the LIS3L02AQ from ST Microelectronics. It is a 3 axis analogue accelerometer with a selectable range of either $2 \mathrm{~g}$ or $6 \mathrm{~g}$ in a QFN-44 package. A test board was constructed to determine whether the accelerometer would be suitable for the ROV. The test board was set up so that the self test (ST), power down (PM) and full scale (FS) settings could be changed simply by altering the jumpers on it.

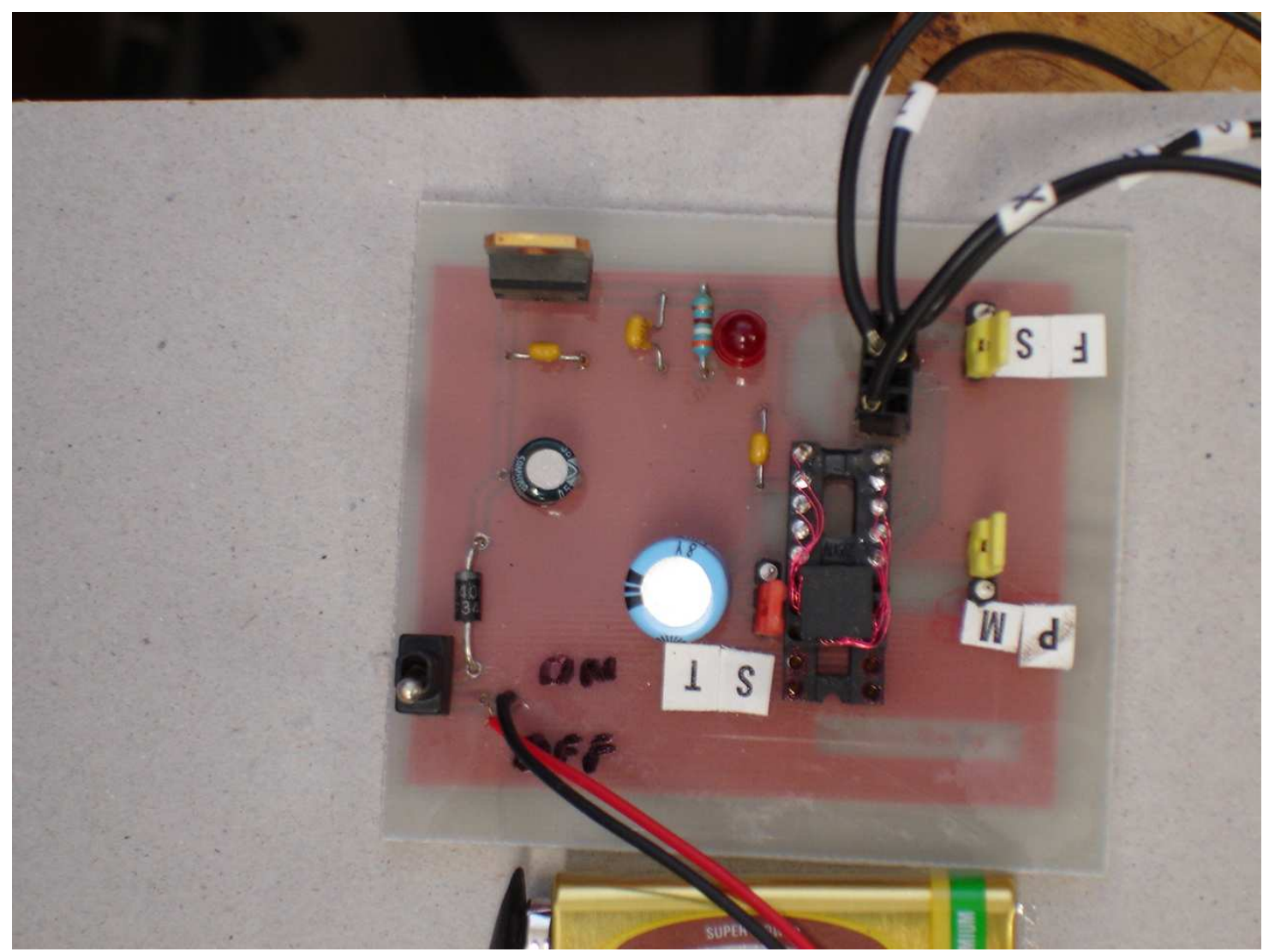

Figure 3.1 LIS3LV02AL Accelerometer 
The self test line applies a voltage into the chip to change the capacitance of the system, giving a measurable output. Depending on the value of the output, it is possible to show whether the chip is malfunctioning. The power mode sets the accelerometer into low power mode, a function primary aimed at mobile or portable systems that do not need to constantly measure the acceleration of the device. For the ROV's purposes the line will be held high so that it does not power down. The full scale jumper selects whether the device is measuring the $6 \mathrm{~g}$ range or the $2 \mathrm{~g}$ range. The ROV's was set at $2 \mathrm{~g}$ because it is unlikely to encounter any acceleration greater than this during normal operation. It also includes a power regulation section to provide a clean $3.3 \mathrm{~V}$ supply for the accelerometer, which comes from a battery.

The outputs from the accelerometer are fed to an IOTech 2005D Data Acquisition (DAQ) (Section 3.3.2) board that is installed in a PCI slot on the computer of the ROV. From the data sheet, the sensitivity is $0.66 \mathrm{~V}$ per g. From Figure 3.2, which was captured from a Fluke 199C Scopemeter, below we can determine that the acceleration sensed was $+0.32 \mathrm{~g},-0.44 \mathrm{~g}$ and $+0.13 \mathrm{~g},-0.27 \mathrm{~g}$ for the red (X direction) and blue (Y direction) waveforms below respectively. The $\mathrm{Z}$ direction can not be measured on the Scopemeter because the Scopemeter has only two input channels. 


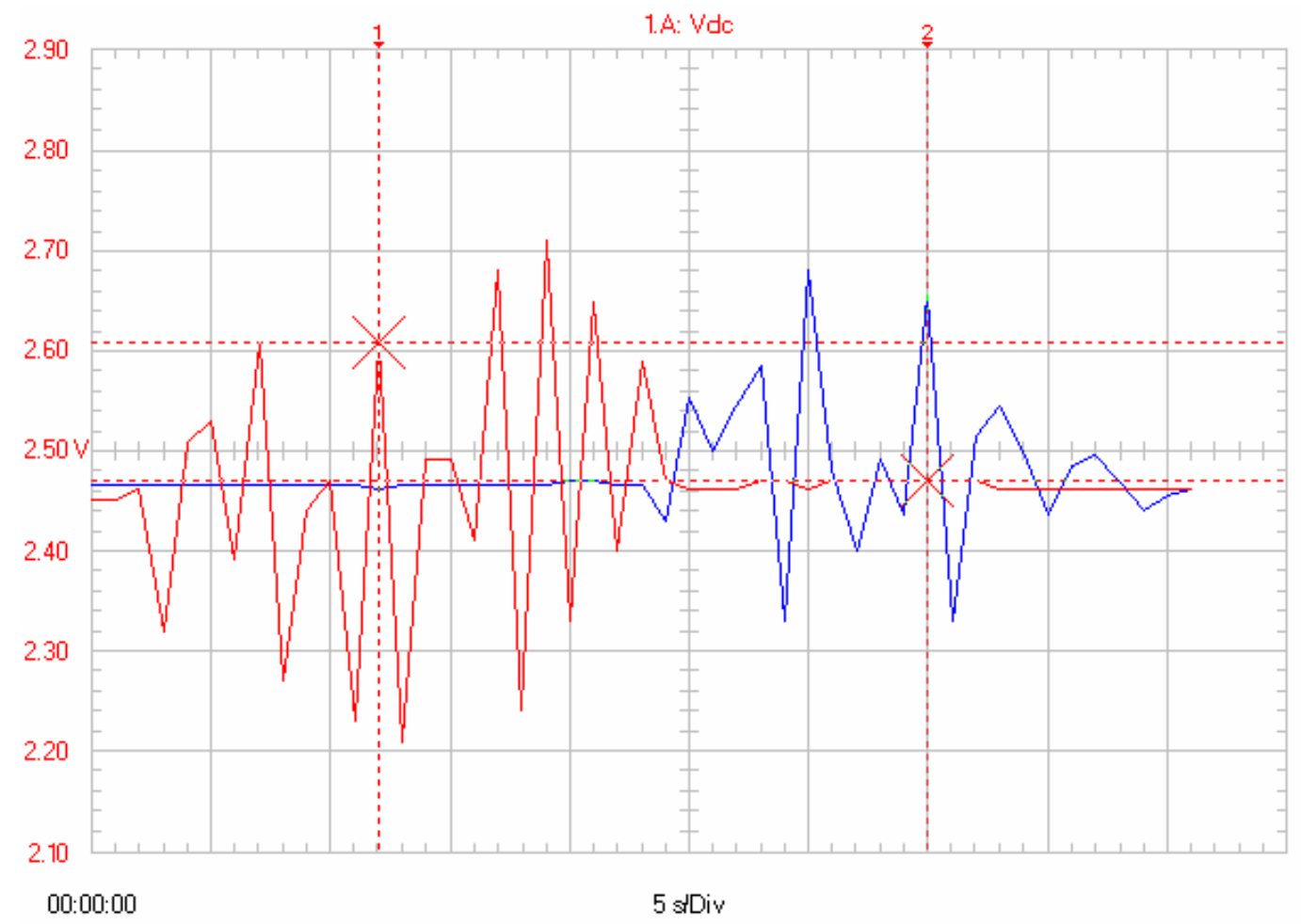

Figure 3.2 Analogue output from LIS3L02AQ3

The readings shown in Figure 3.2 were taken when the initial version of the data sheet was released in 2005, which stated that the device could be run off $5 \mathrm{~V}$. If no acceleration was detected the voltage on the output lines is half $\mathrm{V}_{\text {input }}$ or $2.5 \mathrm{~V}$ as shown above. Subsequence releases of the data sheet changed the maximum input voltage to $3.3 \mathrm{~V}$, which meant that the output from the device, with no acceleration present, was $1.65 \mathrm{~V}$.

This showed an immediate flaw: the lines are highly susceptible to noise. This is a significant problem because the limited room in the ROV means that the analogue lines from the accelerometer are run beside the power lines and other sensor lines. However, a larger noise originates from the motors: the robot is controlled by four industrial $110 \mathrm{~V}$ power drill motors turning propellers. Assuming that the motors only produced noise at $1 \%$ of there full working voltage, this would still create a possible voltage swing of \pm 1.1 $\mathrm{V}$ on any line. Since the mean voltage for the analogue signal is $2.5 \mathrm{~V}$, if $\pm 1.1 \mathrm{~V}$ was introduce onto the same line, the noise would completely destroy the signal from the 
accelerometer unless filtering was carried out before feeding the signal into the DAQ card.

It was decided that digital accelerometers would be better suited for the task than an analogue accelerometer because they are not as susceptible to interference from electrical noise. A new list of required specifications was written up for the new digital accelerometer:

- 3 axis (X, Y and $\mathrm{Z}$ ): all directions need to be measured because the ROV can accelerate in all of these dimensions;

- Relatively high operating temperature range: because of the confined space in the hull and the heat put out by other components it has to be able to with stand these temperatures

- Fast response time: this allows a quicker update rate, which will reduce errors.

- A small package due to space constraints in the ROV.

In the following table (Table 3.1) the following acronyms are used:

LCC - Leadless Chip Carrier

DFN - Dual Flat No Lead

QFP - Quad Flat Package 
Table 3.1 shows a comparison of some of the digital accelerometers available.

\begin{tabular}{|l|l|l|l|l|l|l|l|}
\hline Device & $\begin{array}{l}\text { Input } \\
\text { Power }\end{array}$ & $\begin{array}{l}\text { Acceler. } \\
\text { range }\end{array}$ & $\begin{array}{l}\text { No. of } \\
\text { Axes }\end{array}$ & $\begin{array}{l}\text { Cross } \\
\text { Axis }\end{array}$ & $\begin{array}{l}\text { Non } \\
\text { Linearity }\end{array}$ & $\begin{array}{l}\text { Package } \\
\text { Size }\end{array}$ & $\begin{array}{l}\text { Digital } \\
\text { Interface }\end{array}$ \\
\hline KXP84 & $\begin{array}{l}2.7- \\
5.25 \mathrm{~V}\end{array}$ & $2 \mathrm{~g}$ & 3 & $2 \%$ & $0.5 \%$ & DFN 14 & SPI/I2C \\
max & $\begin{array}{l}2.7- \\
3.6 \mathrm{~V}\end{array}$ & $2 \mathrm{~g}$ & 3 & $4 \%$ & $2 \%$ & QFN 44 & SPI/I2C \\
\hline LIS3L02DQ & $\begin{array}{l}2.16- \\
3.6 \mathrm{~V}\end{array}$ & $2-6 \mathrm{~g}$ & 3 & $3.5 \%$ & $3 \%$ & QFN 28 & SPI/I2C \\
\hline LIS3LV02DQ & $\begin{array}{l}4.5- \\
5.5 \mathrm{~V}\end{array}$ & $2-200 \mathrm{~g}$ & 2 & $2-$ & $0.5-1 \%$ & LCC 20 & $\begin{array}{l}\text { Pulse } \\
\text { Count }\end{array}$ \\
\hline Model 1010 & $\begin{array}{l}2.7- \\
3.6 \mathrm{~V}\end{array}$ & $2 \mathrm{~g}$ & 2 & $2 \%$ & $2 \%$ max & LCC 8 & I2C \\
\hline MXC6202x & $\begin{array}{l}2.7- \\
3.6 \mathrm{~V}\end{array}$ & $2 \mathrm{~g}$ & 2 & $2 \%$ & $1 \%$ & LCC 8 & I2C \\
\hline MXC6202G/M & $\begin{array}{l}3.0- \\
5.25 \mathrm{~V}\end{array}$ & $1.7 \mathrm{~g}$ & 2 & $2 \%$ & $1 \%$ & LCC 8 & PWM \\
\hline MXD2020G/M & $2.7-$ & $2 \mathrm{~g}$ & 2 & $2 \%$ & $0.5 \%$ & LCC 8 & PWM \\
\hline MXD6125G/M & & & & & & & \\
\hline
\end{tabular}

Table 3.1 Accelerometer Comparison Table

Table 3.1 also includes 2 axis devices that could be used to create a unit that measures in all 3 axes. This is achieved by mounting the two of the devices perpendicular to each other. This setup provides some redundancy to one of the axes depending on the how the two accelerometers are set up. With the 2 axis devices the package is, however, a LCC, which is currently unsolderable with the tools available to us. From the 3 axis devices 
they all have similar properties: a $2 \mathrm{~g}$ acceleration range and the SPI/I2C communication interface.

The LIS3L02DQ was chosen from the 3 axis devices because its package is small, it can be powered from a lower voltage supply, it was available immediately, and was within our budget at US\$35.95.

\subsubsection{ST Microelectronics LIS3LV02DQ Revision 0}

The LIS3LV02DQ is one of ST Microelectronics's highest quality accelerometers. It is a 3 axis device that communicates through a SPI bus rather than an I2C bus. As mentioned in section 2.5, this is a better option because it allows for a higher communication rate, which means it can update the robot's position at a faster rate than I2C. It also allows the addition of more sensors on the bus.

Additional benefits are that it comes in a small package, and has a low cross axis sensitivity and non-linearity. As the accelerometer runs off only $3.3 \mathrm{~V}$, the regulation of the voltage will be done on the accelerometer board. This is to limit any noise that could get on to the voltage line, which could damage or corrupt data from the accelerometer chip. Because the microcontroller operates off a $5 \mathrm{~V}$ supply and the accelerometer operates off a $3.3 \mathrm{~V}$ supply there has to be a voltage level shifter in order to communicate between the two.

To shift from the 5V level down to the 3.3V level there are three Fairchild Semiconductor NC7SZ08 2-Input AND gate and these are placed on the Serial Clock, Master Output Serial Input and the Slave Select lines. A 3.3V reference voltage is fed into one input in each chip and the signal from the microcontroller is fed into the other side. These are ANDed together and the result is passed to the accelerometer (Figure3.3). 


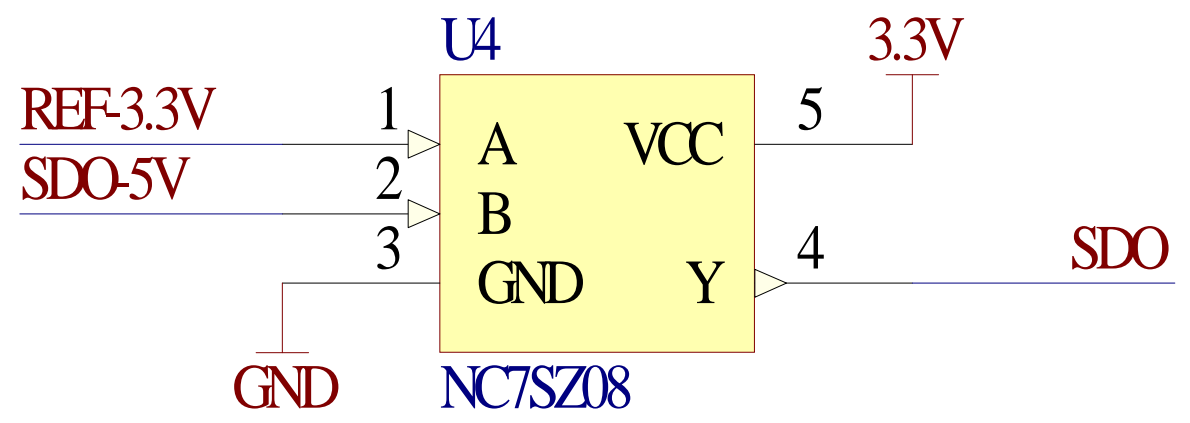

Figure 3.3 Level Shifter for Accelerometer

Once the accelerometer has data that are ready to be transmitted to the microcontroller, the output from the accelerometer needs to be shifted back to $5 \mathrm{~V}$ so that the microcontroller can read the signal. This signal is shifted up using a Texas Instruments SN74AHCT1G08 Single 2-Input Positive AND Gate and a $5 \mathrm{~V}$ reference signal. The accelerometer is connected to the micro with a slotted connecter, which ensures that the plug cannot be connected the wrong way round.

Two capacitors on the power supply remove noise that may have entered the power and ground lines. A LED is also included so that a visual inspection of the device may determine whether it is powered. Figure 3.4 shows the constructed accelerometer board. 


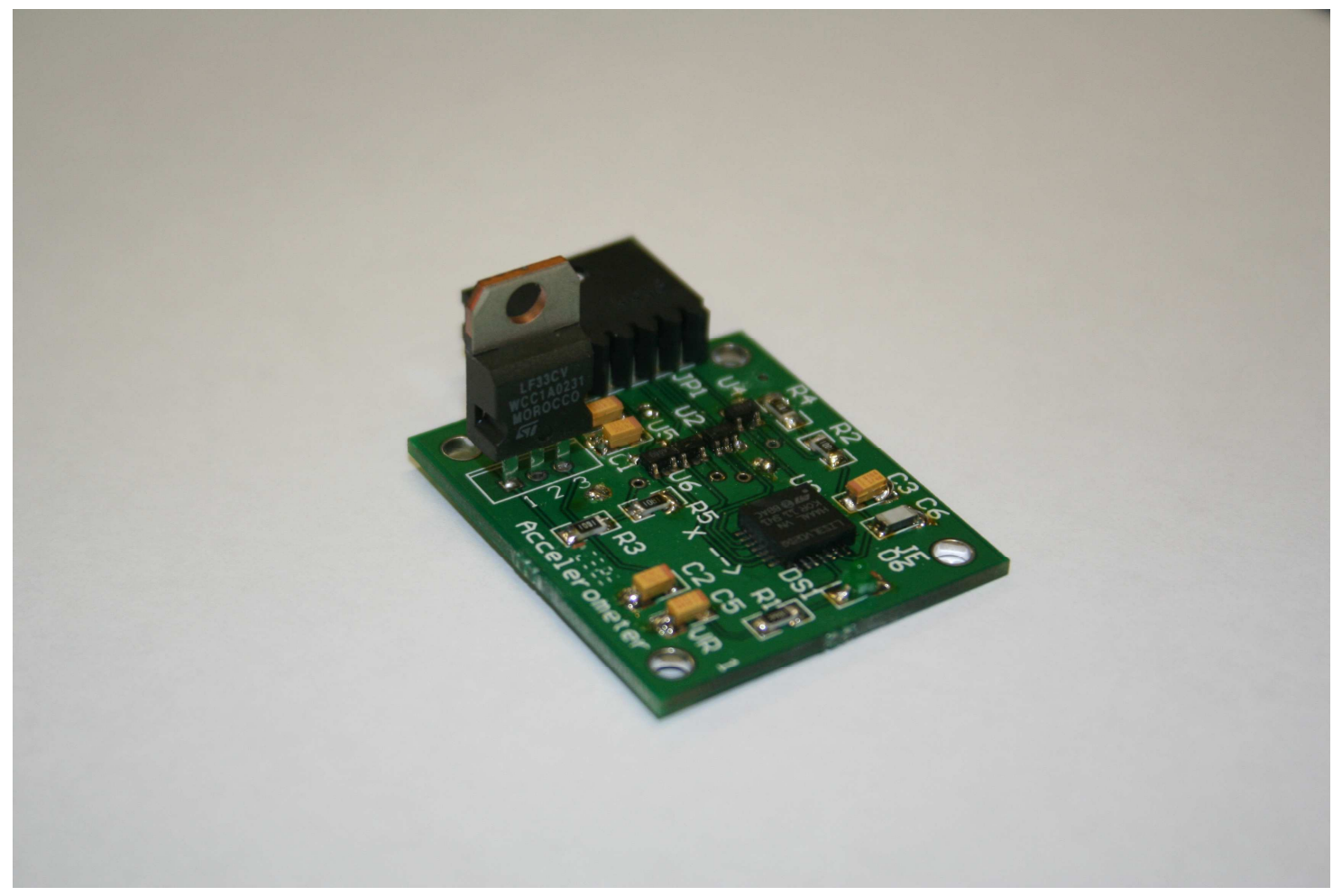

Figure 3.4 Accelerometer PCB

It has a 3.3 voltage regulator to reduce the voltage from the $5 \mathrm{~V}$ that is transferred from the microcontroller board to the $3.3 \mathrm{~V}$ that the accelerometer requires. There are four logic switches, three of them being NC7SZ08's to convert the $5 \mathrm{~V}$ signal into $3.3 \mathrm{~V}$ signals and the final switch being a SN74AHCT1G08 to convert the $3.3 \mathrm{~V}$ output from the accelerometer into a $5 \mathrm{~V}$ signal that the microcontroller can read. The reference signal for each set of switches, the $3.3 \mathrm{~V}$ to $5 \mathrm{~V}$ switch and the $5 \mathrm{~V}$ to $3.3 \mathrm{~V}$ switches, comes from a simple voltage divider.

\subsubsection{LIS3LV02DQ Revision 1}

The first board was redesigned after it was discovered from [21] that if gyroscopes were not included in IMUs, the accelerometers alone could not correctly position the ROV. To correctly map the ROV into the operator's frame of reference, gyroscopes are also required to determine the orientation of the vehicle. This revision includes a gyroscope for yaw direction detection, and also contains connection points so that two additional 
gyroscopes can be added to take into account the pitch and roll of the vehicle. Initially these angular directions are to be measured by the Crossbow CXTA02 tilt sensor that is installed in the sensor hull in the port hull canister. These two readings are connected directly to the vehicle's PC via the DAQ card.

The gyroscope chosen for this revision is an Analog Devices ADIS16080. It is a single axis gyroscope that communicates via the SPI interface, has a +/- 80 degrees a second yaw rate, comes in a small package and operates from a 5V supply (which is supplied from the PC).

The data from the gyroscopes are stored in a 16 bit register on chip: the first two bits are low signals and the following two bits state whether the data in the register are from the temperature sensor on the chip, from the gyroscope, or from one of the chip's two analogue inputs. The remaining 12 bits of register contain the data that are to be sent out with the MSB bit being the highest of the 12 bits.

The gyro is mounted ahead of the accelerometer on the board, and all the power supplies for the board are placed on the bottom of the PCB. The connectors for the communication lines (including the lines for the gyroscopes,) are placed at the far end of the board. 


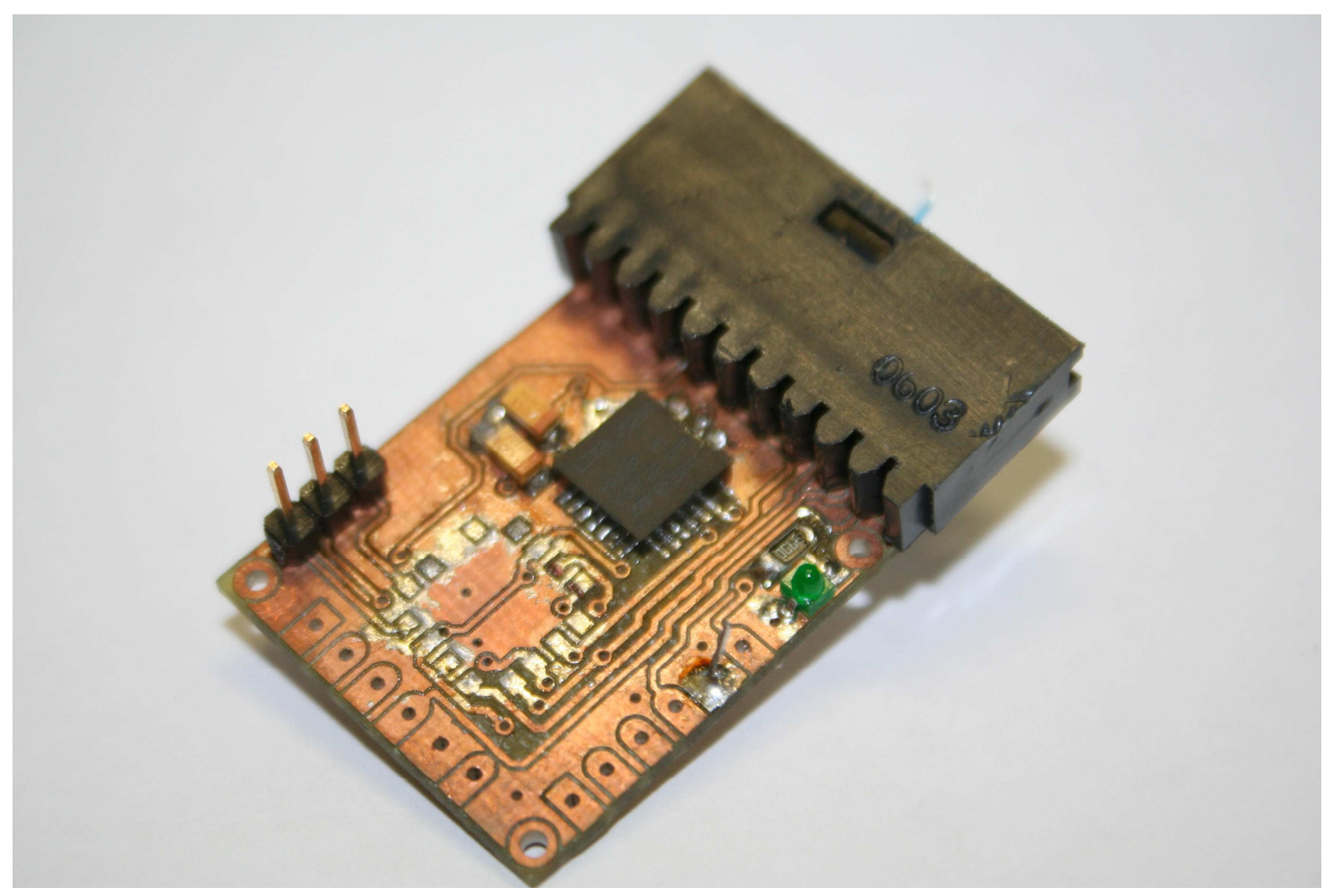

Figure 3.5 LIS3LV02DQ Revision 1

During testing no output could be obtained from this device. The board has no solder mask (Figure 3.5) and has a power line running under the LIS3LV02DQ chip. It was therefore deduced that a possible cause of the lack of output was the creation of a short circuit due to the ground pad on the bottom of the chip. Another possibility was a small short circuit between the pads of the chip and the pads on the printed circuit board. Removal and replacement of the chip would not necessarily prevent this type of problem happening, so it was decided to refine the board again.

\subsubsection{LIS3LV02DQ Revision 2}

To combat the short circuit problems with the accelerometer and gyroscope chips, new boards were required to have solder masks. An accelerometer board was available with the chip pre-soldered from Sparkfun Electronics in the United States of America for US\$39.95. This board is shown in the following Figure 3.6. 


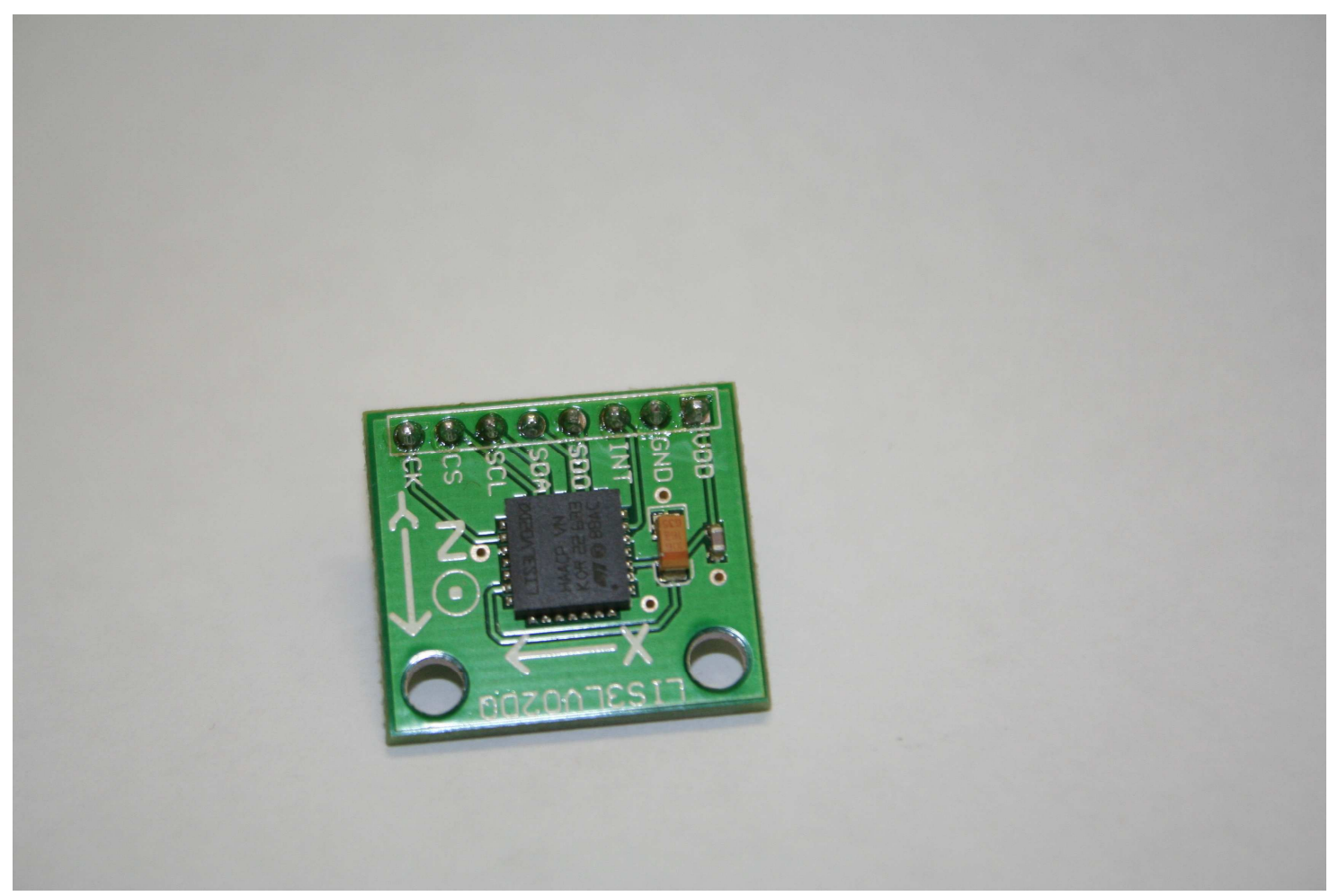

Figure 3.6 LISV02DQ Breakout Board

A simple resistor divider network replaced the AND gate solutions of the previous revisions. This reduced the component count and simplified the unit. Testing of an evaluation design with resistor dividers showed that this new design worked. In the previous revision the power supplied to the accelerometer was from a $3.3 \mathrm{~V}$ regulator that obtained power from the $5 \mathrm{~V}$ line from the microcontroller - this was also replaced with a resistor network to supply $3 \mathrm{~V}$ to the accelerometer. Figure 3.7 shows the latest revision of the IMU printed circuit board. 


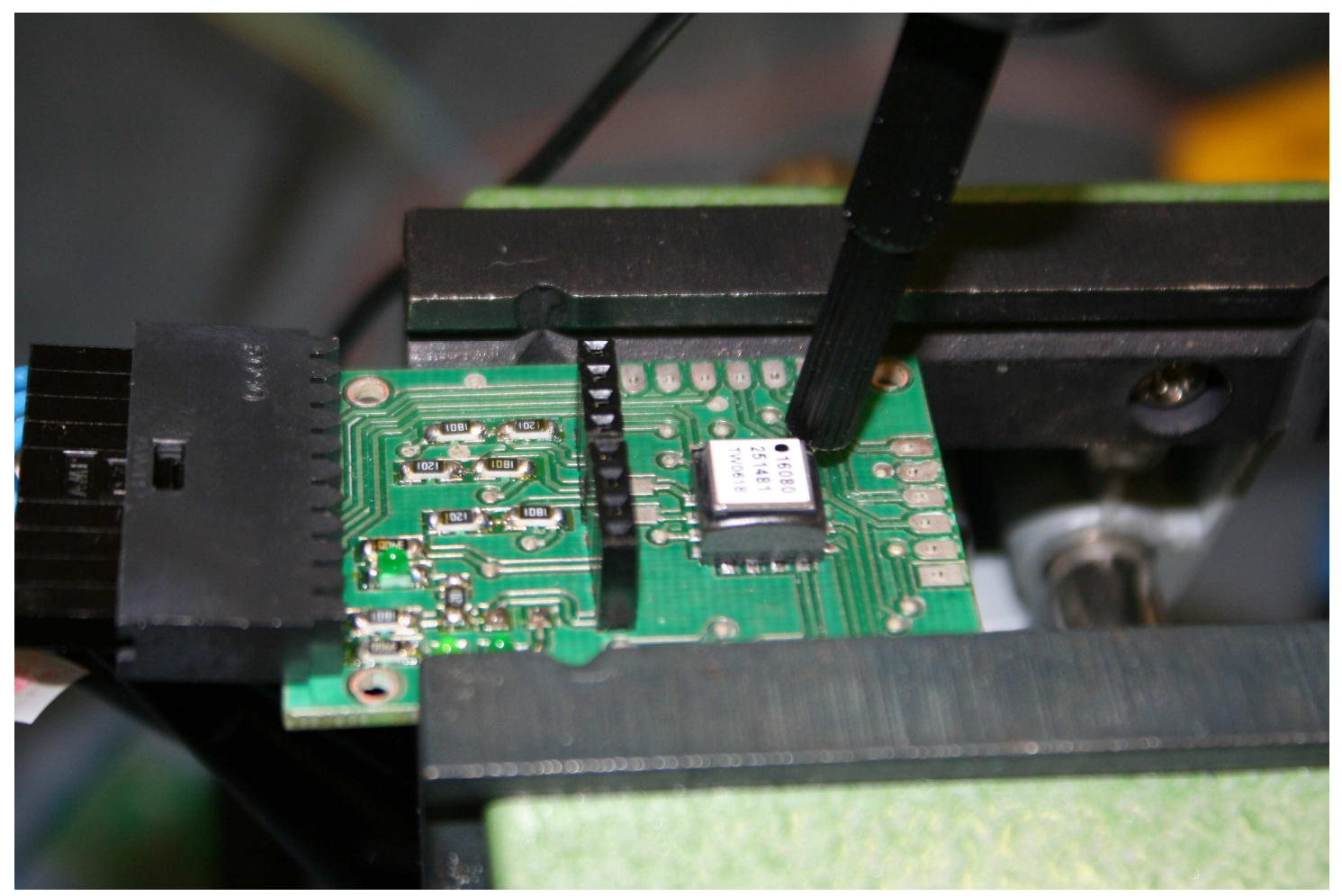

Figure 3.7 LIS3LV02DQ IMU Board Revision 2

Figure 3.7, above shows the new board design. At the left is the connector that connects the IMU board to the micro. Between the micro connector and the vertical connector for the Sparkfun LIS3LV02DQ accelerometer breakout board is the resistor divider network. This drops the signal voltage down from $5 \mathrm{~V}$ to the $3 \mathrm{~V}$ the accelerometer requires so it does not damage the device. There are also three LEDs located on the board. The one in the lower left of the board is the $5 \mathrm{~V}$ status LED, the one next to it is the $3 \mathrm{~V}$ status LED and the last one is the data ready LED. The data ready LED will only work if the accelerometer has been set up to send an interrupt signal back to the micro when data are ready to be transmitted.

Initially the gyroscope worked by itself as shown in the Figure 3.8. The data sent to the Gyro is the yellow waveform with the green waveform being the returning data. The purple waveform is the clock signal from the microcontroller which controls the clocking of the data with the chip select signal in blue. 
Hardware 55

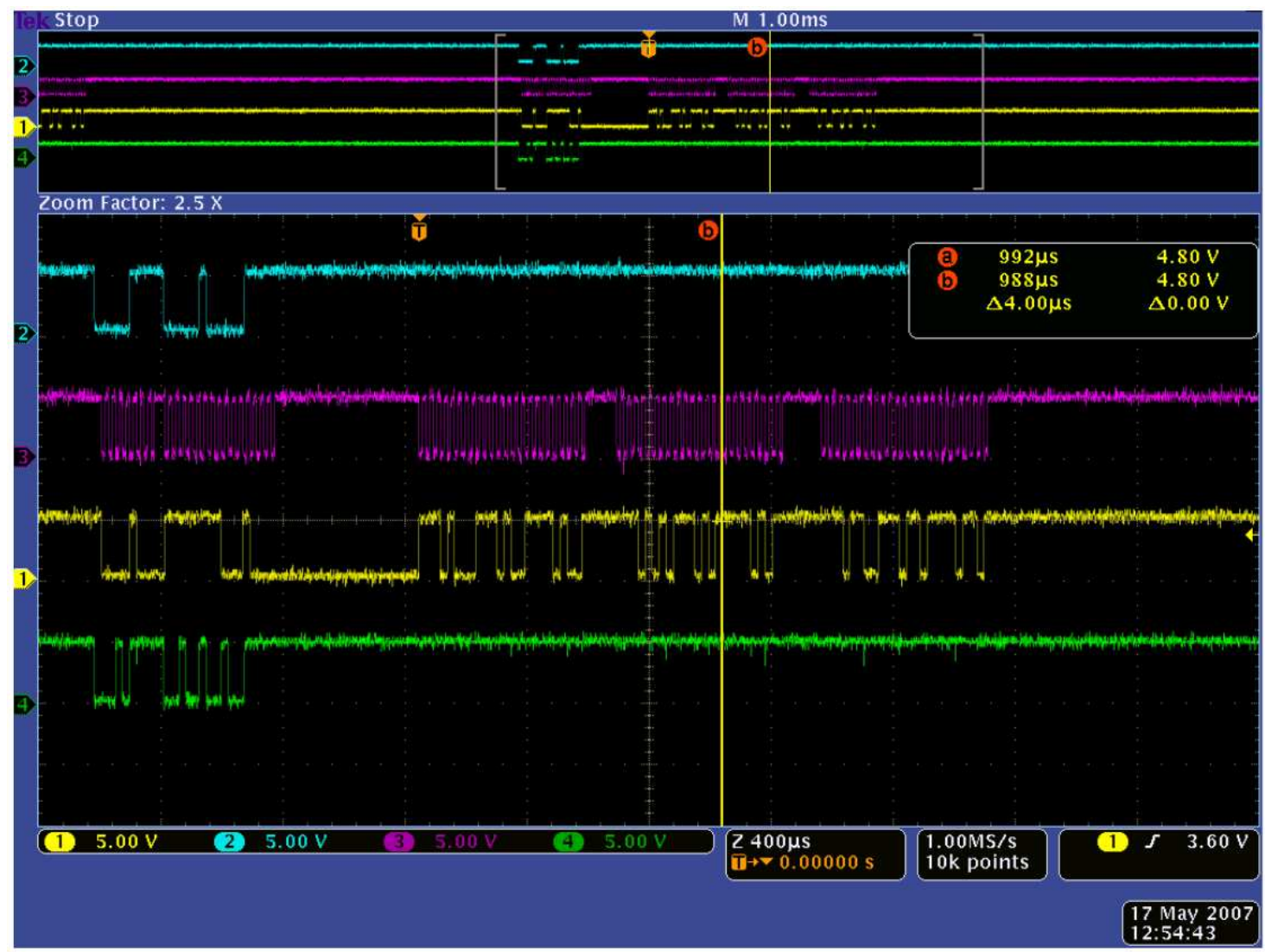

Figure 3.8 Gyroscope Data Transmission

However, problems were encountered when the accelerometer was added to the IMU board. Once the board was added the signals from both chips stopped communicating with the micro. Testing the lines revealed a possible cause: varying voltage on the MISO line. When the gyroscope was sending data to the micro, the voltage on the MISO line was $5 \mathrm{~V}$ and when the accelerometer was sending data on the MISO line, the voltage on the line was $3 \mathrm{~V}$. Because there is no buffer between the differing voltages, the $5 \mathrm{~V}$ from the gyroscope has the potential to enter the output pin of the accelerometer. While this is within the absolute maximums of the LIS3LV02DQ, it would nevertheless degrade the performance of the accelerometer.

Another problem that occurred was that the chip select line for the accelerometer was not being seen as zero. The accelerometer transmits and receives data when its chip select line is low, otherwise it ignores any data on the SPI lines. When the breakout board was plugged in, the zero voltage level was shown on the scope to be $2.6 \mathrm{~V}$, which is not low 
enough to be seen as a zero signal on the accelerometer. The data sheet for the accelerometer shows that the maximum value for a low signal on the accelerometer is given by the following formula:

$$
0.2 * \text { VDD_IO }
$$

VDD_IO is the voltage that drives the input and output lines of the accelerometer. Since the accelerometer's VDD_IO drive pin is running off $3 \mathrm{~V}$, this gives a maximum value of $0.6 \mathrm{~V}$ for a low signal. The minimum for a high signal is given by the following formula:

$$
0.8 * \text { VDD_IO }
$$

This means the minimum high signal voltage level needs a voltage of $2.4 \mathrm{~V}$, as seen by the accelerometer. Since the scope showed the zero level as $2.6 \mathrm{~V}$, as far as the accelerometer is concerned the chip is not selected.

\subsubsection{LIS3LV02DQ Revision 3}

To stop the differing voltages damaging the separately powered devices a solution was found using an n-channel MOSFET. The design is from an application note used on I2C lines to communicate between two different voltage levels.

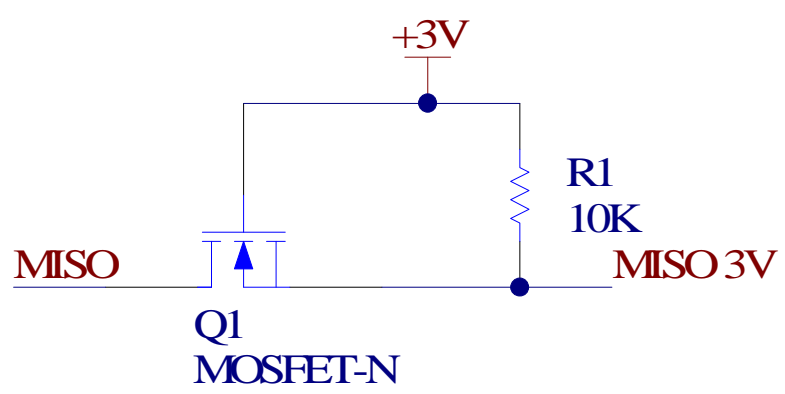

Figure 3.9 MOSFET Signal Circuit 
As can be seen in the schematic in Figure 3.9, when the accelerometer outputs a logic high signal, the output line to the MOSFET, the gate to source voltage $\mathrm{V}_{\mathrm{GS}}$ of the FET is $0 \mathrm{~V}$. This turns the MOSFET off and the $5 \mathrm{~V}$ line that is connected to the drain is pulled up by the microcontroller's internal pull up resistor. When there is a logic low on the accelerometer MISO line, $\mathrm{V}_{\mathrm{GS}}$ is $3 \mathrm{~V}$, which turns the device on, and the drain is pulled down to the voltage level of the source, which is at $0 \mathrm{~V}$.

The voltage levels on the chip select line for the accelerometer were corrected by using a Schmitt trigger to buffer the signal from the micro to the accelerometer. Because the Schmitt trigger inverts the signal, the output is fed back into another Schmitt trigger, which just acts as an inverter to restore the original orientation. This will replace the voltage divider network that was used in previous versions.

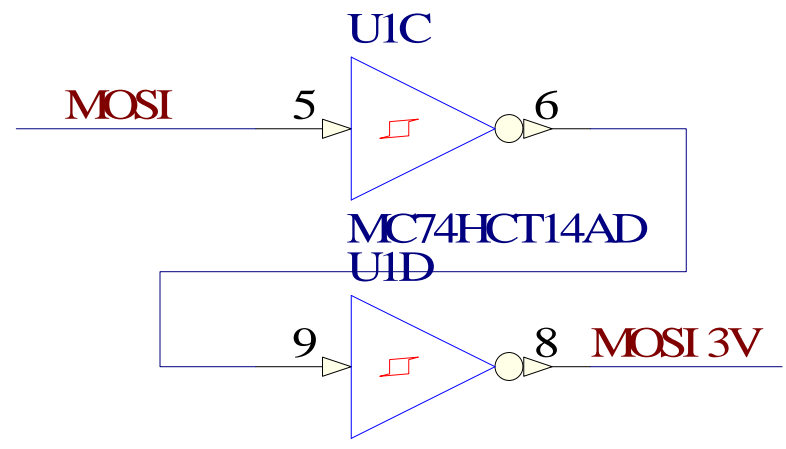

MC74HCT14AD

Figure 3.10 Schmitt Trigger Layout

The Schmitt trigger is applied to the CS, MOSI, and SCLK line going into the accelerometer, which is easily implemented as the Schmitt trigger and inverters come in multiple device packages. 


\subsection{Other Hardware}

\subsubsection{The On Board Computer}

The computer in the ROV must be able to handle the complex tasks of data gathering and computer intensive calculations based on these data, to enable the user on the surface to accurately control the robot for its given task. At the start of the project it was decided to upgrade the computer, since the software being used on the robot was going to be upgraded as well. The use of a PC computer in the ROV means that 'off the shelf' components and software can be used. An example is the camera in the main hull, which is a web camera that has been stripped down to fit the mount [22].

The size constraints of the ROV's main hull limit the size and space the computer can safely take up in the main hull of the robot. The main hull is an aluminium cylinder with a depth of $160 \mathrm{~mm}$ and a diameter of $355 \mathrm{~mm}$. This space must contain the computer motherboard, the computer hard drive, the power supply for the computer, the Data Acquisition card (DAQ) (which will read in information from the sensors), the micro controller board (to communicate between the motors and the PC), the temperature, humidity and water alarm sensors [23], the video camera (to allow the operator a view of any features in front of the robot) and the Inertial Navigation System.

The previous computer in the robot had the following specifications
- Gigabyte GA-5VMM Micro ATX form factor motherboard
- AMD $500 \mathrm{MHz}$ K62 processor
○ 128 Mbytes of RAM
○ D-Link DE-528 PCI Ethernet card
○ 14.3 GB Hard Drive 
The DAQ is a National Instruments Lap-PC+ card that was installed into one of the PCI slots on the motherboard. This card has eight single channel analogue inputs, three digital ports and two 12 bit digital to analogue outputs. It is installed in the ISA slot in the motherboard.

The new computer must have the same or smaller physical dimensions (due to the limited space). A Shuttle XPC system was chosen to be the new computer for a number of reasons including its form factor, which is similar to the old PC's Micro ATX form, its processing capabilities, and its power supply size. The power supply size is very important because the previous computer's power supply was a standard ATX power supply that had been cut to fit inside the hull (Figure 3.11). With the shuttle computer, the power supply is already designed to fit into a tight space and therefore it can be easily placed inside the hull (Figure 3.12).

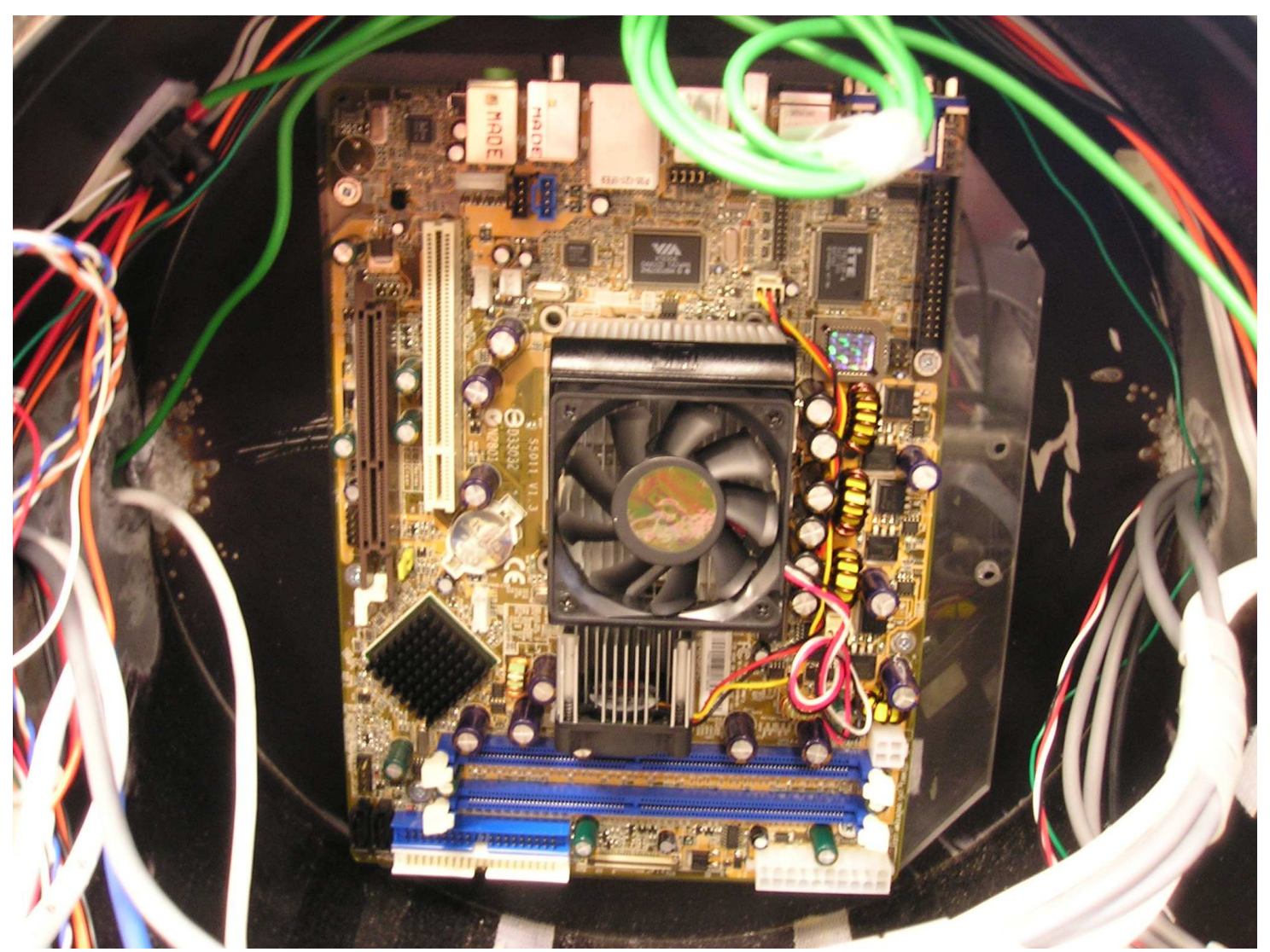

Figure 3.11 Mock Up Shuttle PC Motherboard Showing the Size Constraints of the Main Hull 


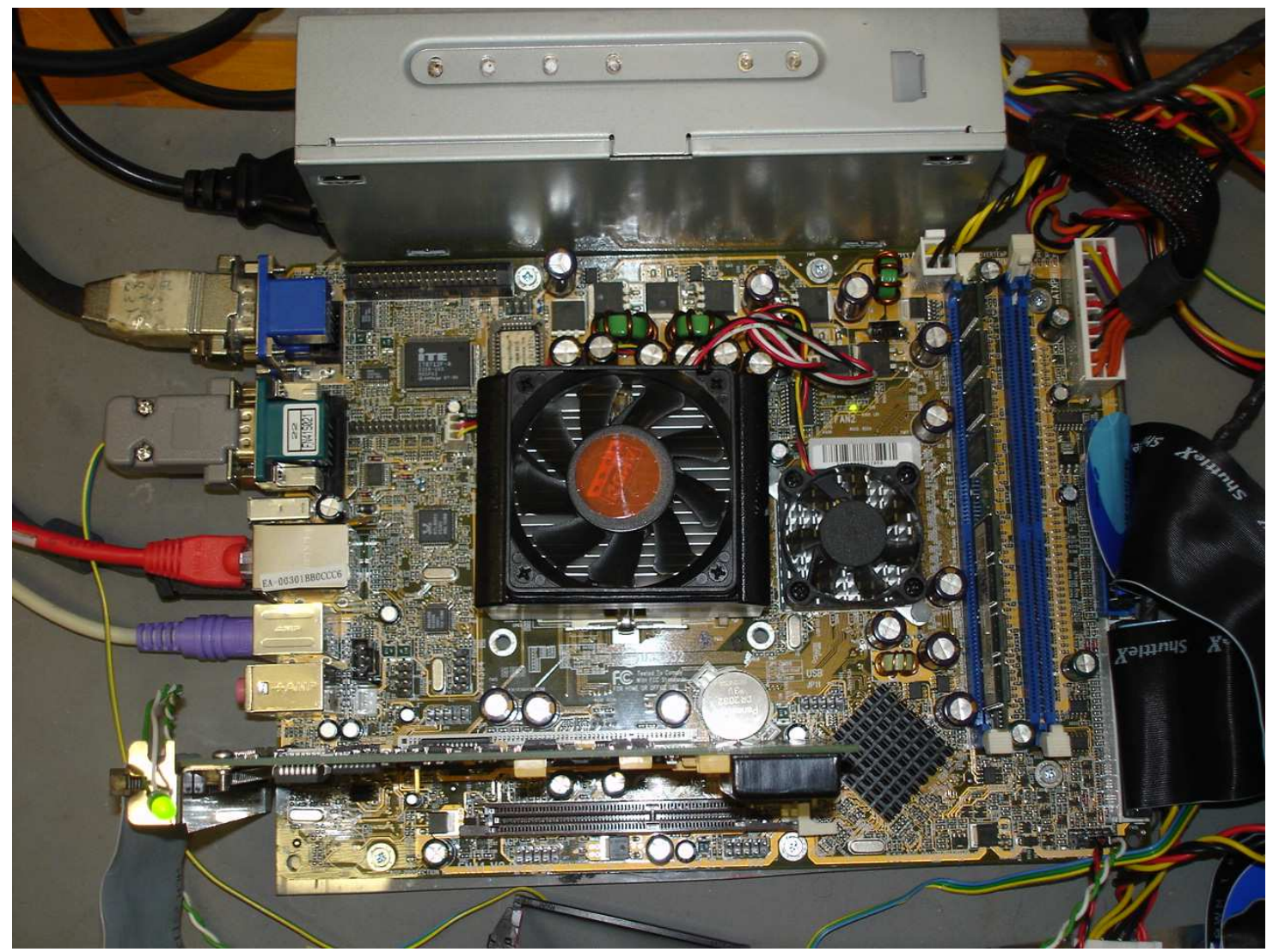

Figure 3.12 The PC with all the Ancillary Parts Connected

The new system has the following specifications:

○ Shuttle SN41 V2.0 motherboard $(25.4 \mathrm{~cm} \times 18.5 \mathrm{~cm})$

○ AMD Sempron $2400 @ 1.19 \mathrm{GHz}$

- 1 Gigabyte of RAM (2× 512 Megabyte sticks)

- A 230 Watt shuttle power supply

- Seagate 160 GB ATA Hard drive

The previous computer was mounted inside the hull using an acrylic backing plate held in place with industrial strength Velcro. By using the shuttle motherboard it was possible to reuse these parts; this also gave us room to mount the power supply on the side. The acrylic backing plate was tapped to match the mounting positions of the Shuttle motherboard and the motherboard was then screwed into place. 
To stop the DAQ card falling out of the PCI slot while the ROV is in use, a small metal bracket has been made to hold the card in place. The DAQ card is screwed into the acrylic backing plate through the backing tab. The small metal bracket fits over the top edge of the card at the far end from the backing tab. This bracket is connected to a threaded rod that runs from the backing plate, through one of the motherboard's mounting holes, and upwards to the bracket as shown in Figure 3.13.

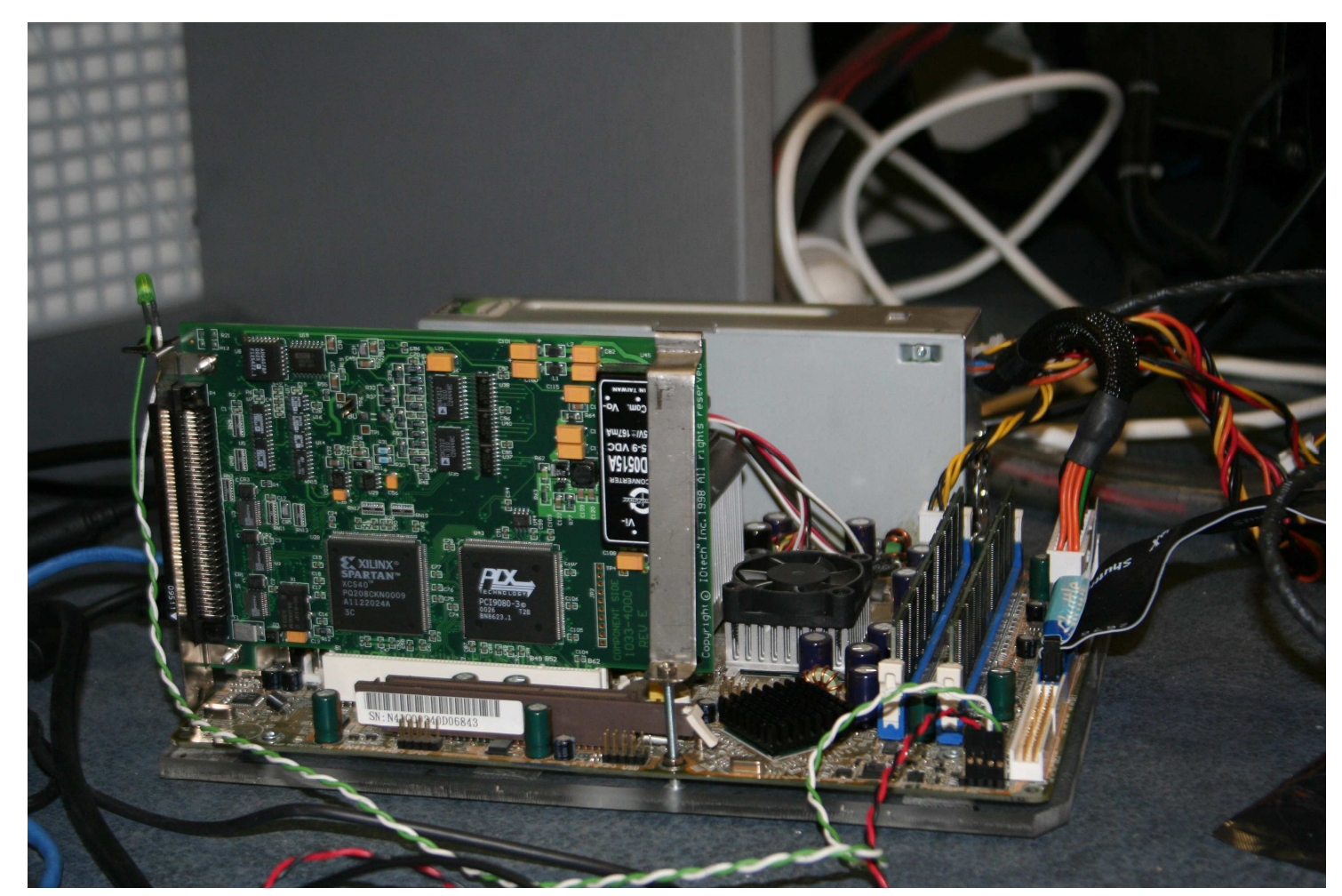

Figure 3.13 DAQ Card Support Bracket

The hard drive is attached to the back of the hull with industrial Velcro just like the motherboard, with a small piece of acrylic screwed onto the hard drive. The threads in the hard drive were tapped to M4 size because the thread size that most hard drives have for mounting holes (5/16") is hard to source, so the same size holes could not be drilled into the acrylic. The hard drive is held in place with $10 \mathrm{~mm}$ M4 screws that have been cut down to $8 \mathrm{~mm}$ to fit the mounting depths of the holes in the hard drive. 
The network cable that ran to the original motherboard was a co-axial cable that is incompatible with the new motherboard, so it had to be replaced. This required the removal of all the oil in the electrical tubing (the oil is used to keep water out of the connections going into the main hull of the ROV). Once this had been removed, the junction box where all the electrical cables met (before going out to become the ROV's umbilical) was unbolted. The mould that held the umbilical together before being fed out a waterproof conduit was cut apart to separate the three power cords: one for the motors, one for the sensors, and one for the ROV's computer and the co-axial network cable. The co-axial cable connector was then cut at the PC end and the plug at the entrance to the main hull was split. The cable was pulled backwards through the junction box and out of the ROV, and then discarded.

The replacement cable for the ROV is a Category 5 network cable. This cable has better data rates than co-axial cable, and can be used for much greater distances without signal degradation as shown in the following table.

\begin{tabular}{|c|c|c|}
\hline & Category 5 & Co Axial \\
\hline Duplex & Full & Half \\
\hline $\begin{array}{c}\text { Transmission } \\
\text { length }\end{array}$ & Up to $100 \mathrm{~m}$ & Up to $180 \mathrm{~m}$ \\
\hline Cable Diameter & $5 \mathrm{~mm}$ & $10.3 \mathrm{~mm}$ \\
\hline
\end{tabular}

Table 3.2 Network Cable Comparison 


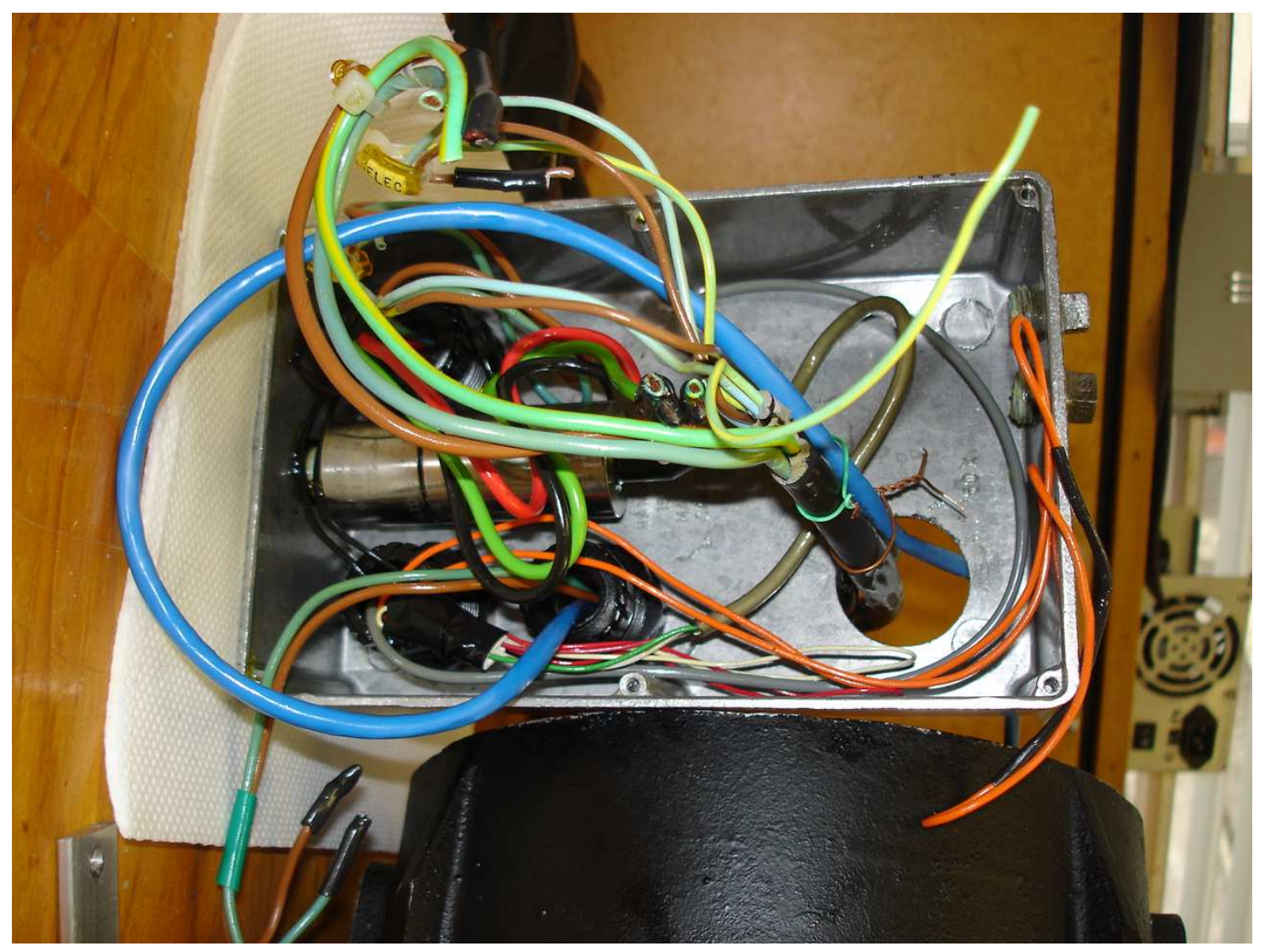

Figure 3.14 Junction Box with new Ethernet cable

The new network cable was measured out to be approximately $25 \mathrm{~m}$ long, then fed into junction box (shown in Figure 3.14), the electrical tubing, and into the main hull. To secure the three power cables and the network cable together at the junction box, and to keep water out and the oil in the junction box, the cables were moulded with 3M's Scotchcast. This compound is specifically designed for electrical applications (with a safe voltage rating up to $4 \mathrm{KV}$ ) and for use underground and underwater. It is a two part epoxy that hardens when the two parts are combined. This was poured into a 100 cubic cm plastic syringe, which was used as the shell for the mould. The compound sets in about three hours and completely hardens in 12. Scotchcast was also used at the point where the cables from the junction box entered the main hull.

Once the compound had hardened, the syringe was split and the mould fed through the umbilical gland into the junction box. The box was reassembled and reattached to the 
ROV chassis. The ends of the cables were then fitted with the RJ45 connectors and the cable was tied together with the three power cables to reform the umbilical.

\subsection{The DAQ Card}

A new data acquisition card (Figure 3.15) was also placed in the computer. This card is from IOTech and has the following specifications:

- 16 single ended or 8 differential ended analogue inputs at $200 \mathrm{kHz}$

- 40 digital IO lines

- Four 16 bit counters and two 16 bit timers

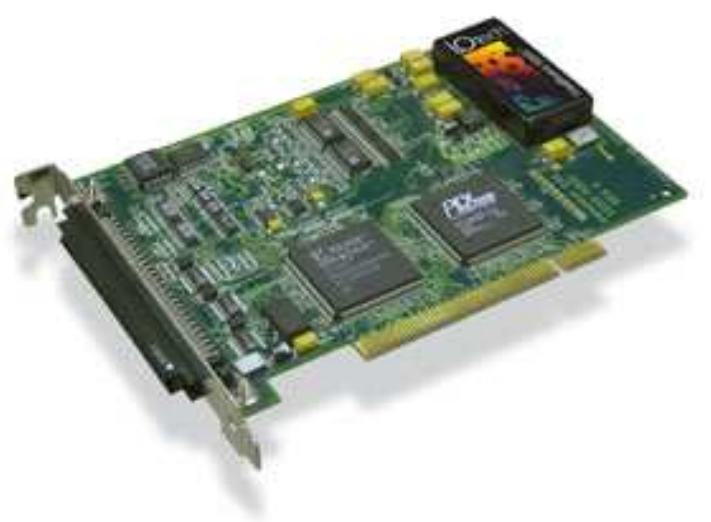

Figure 3.15 DAQ2005 Card

The inputs to the DAQ card are connected through a 100 line ribbon cable that mates with an IOTech DBK 9 connector block. This block has three 39 pin headers taken out to $2.54 \mathrm{~mm}$ pitch screw terminals. The connector block is shown below in Figure 3.16. It was constructed by Luke Cawley, initially for the outdoor mechatron the Tank [24]. 


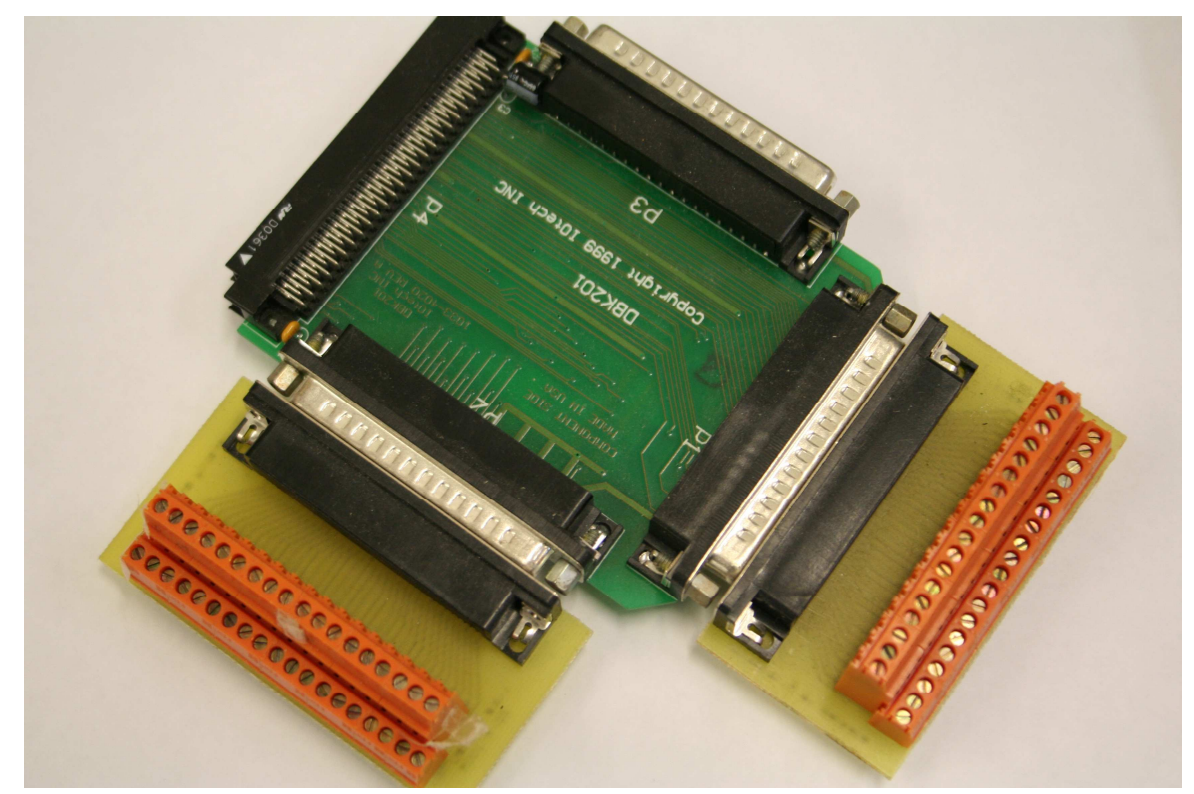

Figure 3.16 DAQ Card Connector Block

\subsubsection{C51 Microcontroller Revision 0}

The microcontroller was upgraded to allow it to communicate with the accelerometer using the SPI interface as well as carrying out the tasks of the original microcontroller. An Atmel T89C51RD2 previously controlled the motors after receiving commands from the computer via a serial port.

The Atmel T89C51RD2 has:

○ 80C52 compatibility - this instruction set is compatible with 8051

○ Four 8 bit I/O ports

○ Three 16 bit timers

○ 256 bytes of scratch pad RAM

○ 64 Kbytes of on chip flash memory

○ On chip 1024 bytes Expanded RAM.

○ Pulse Width Modulation (PWM)

○ Full duplex enhanced UART 
The new microcontroller had to have as many of these functions as possible. It was decided to select the same microcontroller that is used in the undergraduate teaching laboratories at Victoria University of Wellington, which has similar specifications to the T89C51RD2: the Atmel AT89C51AC3. This microcontroller has the following specifications:
○ 80C51 Core
○ 256 bytes of on chip RAM
- 2048 bytes of on chip ERAM
○ $64 \mathrm{~K}$ of on chip Flash Memory
○ Three 16 bit Timers
- Full duplex UART
○ Five ports: $32+4$ digital I/O lines
○ 10 bit ADC
○ SPI interface
○ PWM 


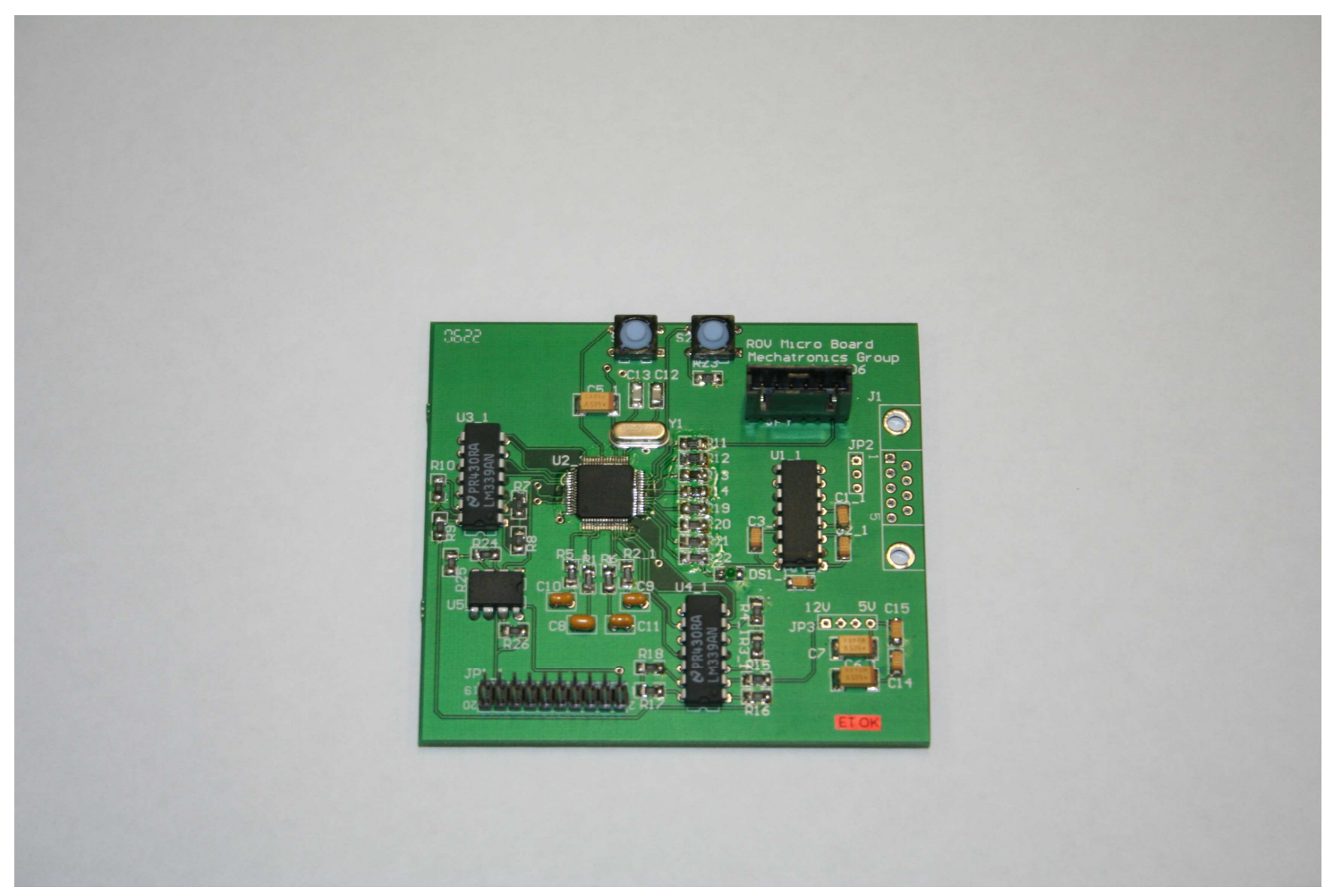

Figure 3.17 Microcontroller Board

The microcontroller (Figure 3.17) has two different power supplies $-5 \mathrm{~V}$ and $12 \mathrm{~V}$. These power lines are provided by connecting the microcontroller to the spare power lines from the PC's power supply unit. Motherboards are not designed to handle large changes in voltage so there should be very little voltage swing in the power supply. However, capacitors are still used to smooth any noise that may reach the lines.

Circuit protection is included in the cable that runs from the Molex connectors from the PC power supply to the microcontroller board. This consists of a $500 \mathrm{~mA}$ resettable fuse and a 5.6 V Zener diode. Once a Zener diode exceeds the Zener voltage it is working in the Zener region which means that the voltage across the diode is held constant even if the current increases. This protection scheme protects the microcontroller board's current supply, with the resettable fuse, and its voltage supply with the Zener diodes. 
This arrangement is also included for the $12 \mathrm{~V}$ line with a $3 \mathrm{~A}$ resettable fuse and a $13 \mathrm{~V}$ Zener diode. The $12 \mathrm{~V}$ line is used to control the motor driver board and has a larger fuse since the motors will draw more current. The microcontroller is not likely to draw anymore than $500 \mathrm{~mA}$.

One of the first problems encountered was that the PSEN line would not go to ground or $0 \mathrm{~V}$, which is required in order to force the microcontroller to enter its bootloader mode. Testing identified that the switches were the cause of the problem. The switches, made with a poly-silicon plunger between the internal contact pads, had a resistance of $40 \mathrm{ohms}$ when closed, which affected the voltage presented to the PSEN line. Replacing these with metal plate mechanical switches that had a closed resistance of 0.2 ohms solved the problem and enabled the bootloader mode to be entered.

Another problem encountered was the switching amplitude of the serial port. The output from the RS232 chip had a low value of $-1.5 \mathrm{~V}$ and a high value of $3.5 \mathrm{~V}$. The output that was expected to be seen on the output from the RS232 on the computer side was $+10 \mathrm{~V}$ and $-10 \mathrm{~V}$. The problem was traced to the charge pump of the RS232 chip using polarised $0.1 \mu \mathrm{F}$ capacitors, which were specified on the data sheet. Once these were changed to non-polarised capacitors of the same value the charge pump functioned as expected.

\subsubsection{C51 Microcontroller Revision 1}

With the addition of Gyroscopes to the IMU, the microcontroller board had to undergo some small changes to allow it to interface with them. The Gyroscopes used the SPI lines to send the data signals, but each gyroscope needed its own chip select line. This necessitated the inclusion of three more lines from the microcontroller as chip select lines. A fourth line was included with this group as the data ready signal from the accelerometer: this tells the microcontroller when new data are available to read, rather than constantly sending signals to the microcontroller. 
An Auto In System Programming (ISP) interface was also included in the hardware, so that the operator can reprogram the microcontroller without having to push and hold the program and reset buttons which drive the PSEN and RESET lines of the microcontroller respectively (Figure 3.18). A signal is sent down the other available lines of the serial port, and this forces the transistors to pull the line low in the case of the PSEN line, and high for the RESET line.

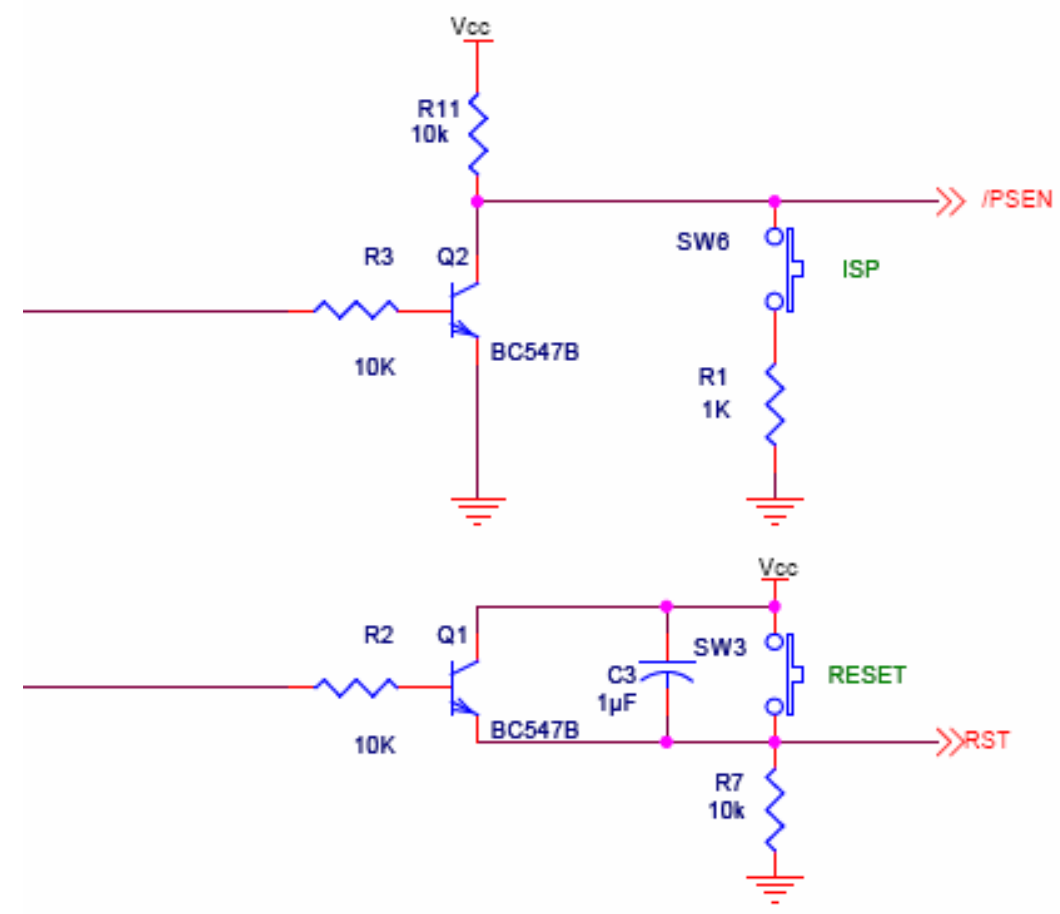

Figure 3.18 Auto ISP Interfacing

Having an ISP means that the vehicle need not be hauled to the surface and taken apart to reprogram. Rather the microcontroller in the ROV may be reprogrammed with new code or reprogrammed with the current program while underwater.

The signals that drive these transistors must first be shifted from the serial values of +10 $\mathrm{V}$ and $-10 \mathrm{~V}$ into the $5 \mathrm{~V}$ signals that the microcontroller requires. This is done by passing the signals down an additional two receive lines in the serial chip. The original 
MAX202 chip on the board had two transmit and two receive lines, and thus was unable to handle the extra lines in addition to the original transmit and receive lines from the microcontroller. The Max202 was replaced with a Sipex 3282 serial chip that has three receive and five transmit lines, which enables the Auto ISP to be included. LEDs for the program, reset, serial transmit, and serial receive were also added to aid debugging when the micro is not connected to the ROV's computer (Figure 3.19).

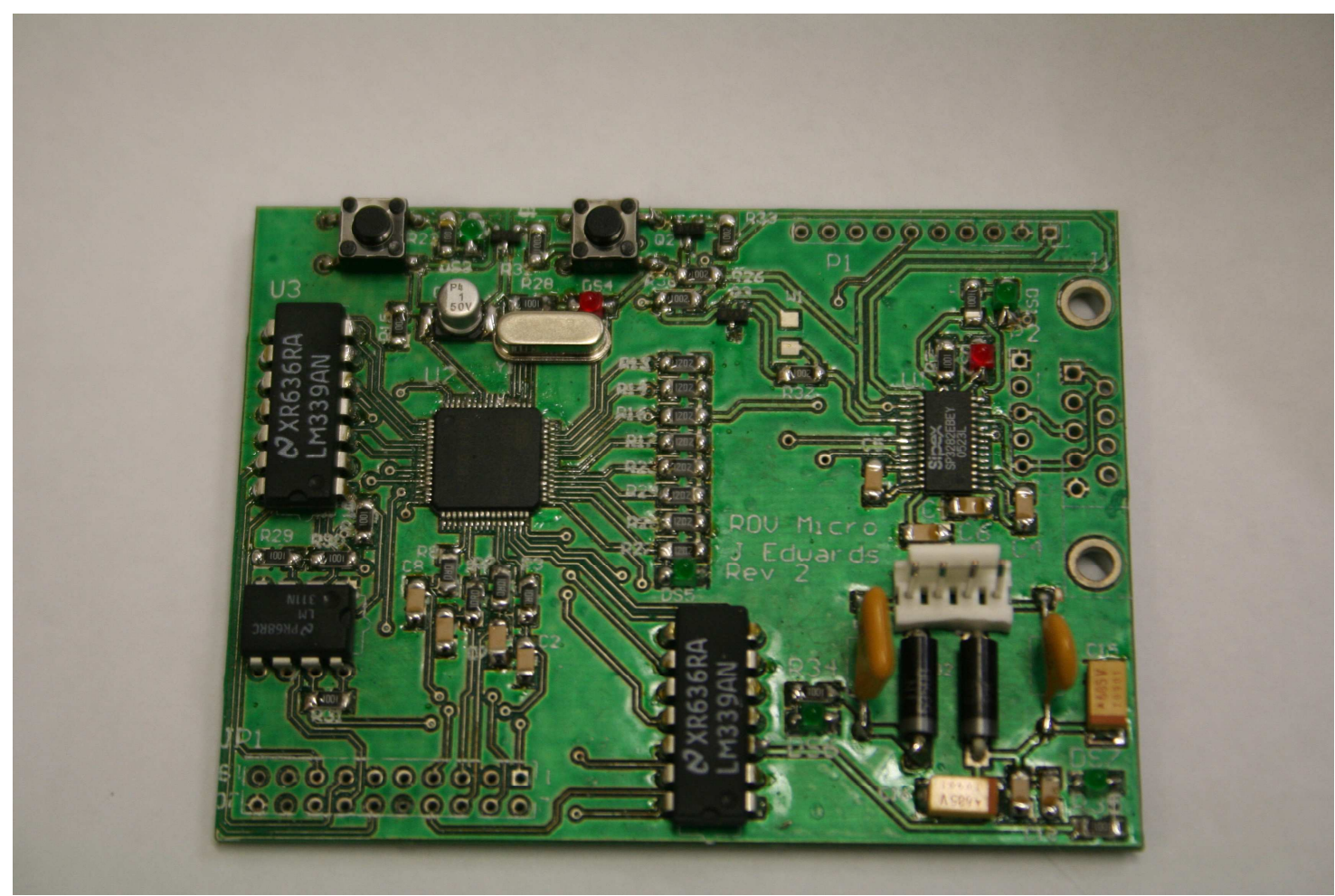

Figure 3.19 Microcontroller Board Revision 1

\subsubsection{Hull Modifications}

One of the smallest changes to the vehicle chassis itself relates to getting access to the main hull. Previously getting into the robot's hull required the removal of the umbilical and cable anchor point, which had to be unbolted from chassis. Then the buoyancy block had to be unbolted and lifted off the robot. Finally the bolts to the front hull could be accessed to remove them to get into the hull. The entire process took at least an hour. 
The three bolts that were used to secure the main hull together on the front hull passed through two flanges: one on the main chassis and one on the front hull piece. The hull is aluminium to limit corrosion the robot may suffer as a result of contact with seawater. The bolts are steel, and the threads of the flanges on the main chassis and the hull have been stripped due to steel wearing into the aluminium from the constant need to get into the hull.

To remedy this situation some nuts were screwed on to the back of each bolt to hold them in place. These bolts have also had their threads damaged by the aluminium chassis. To make it easier to remove the front hull, all the bolts were replaced and helicoil inserts (Figure 3.20) installed to ensure that the bolts did not wear down the chassis again. The inserts are fitted in the flanges between the chassis and the point at which the bolt would screw in.

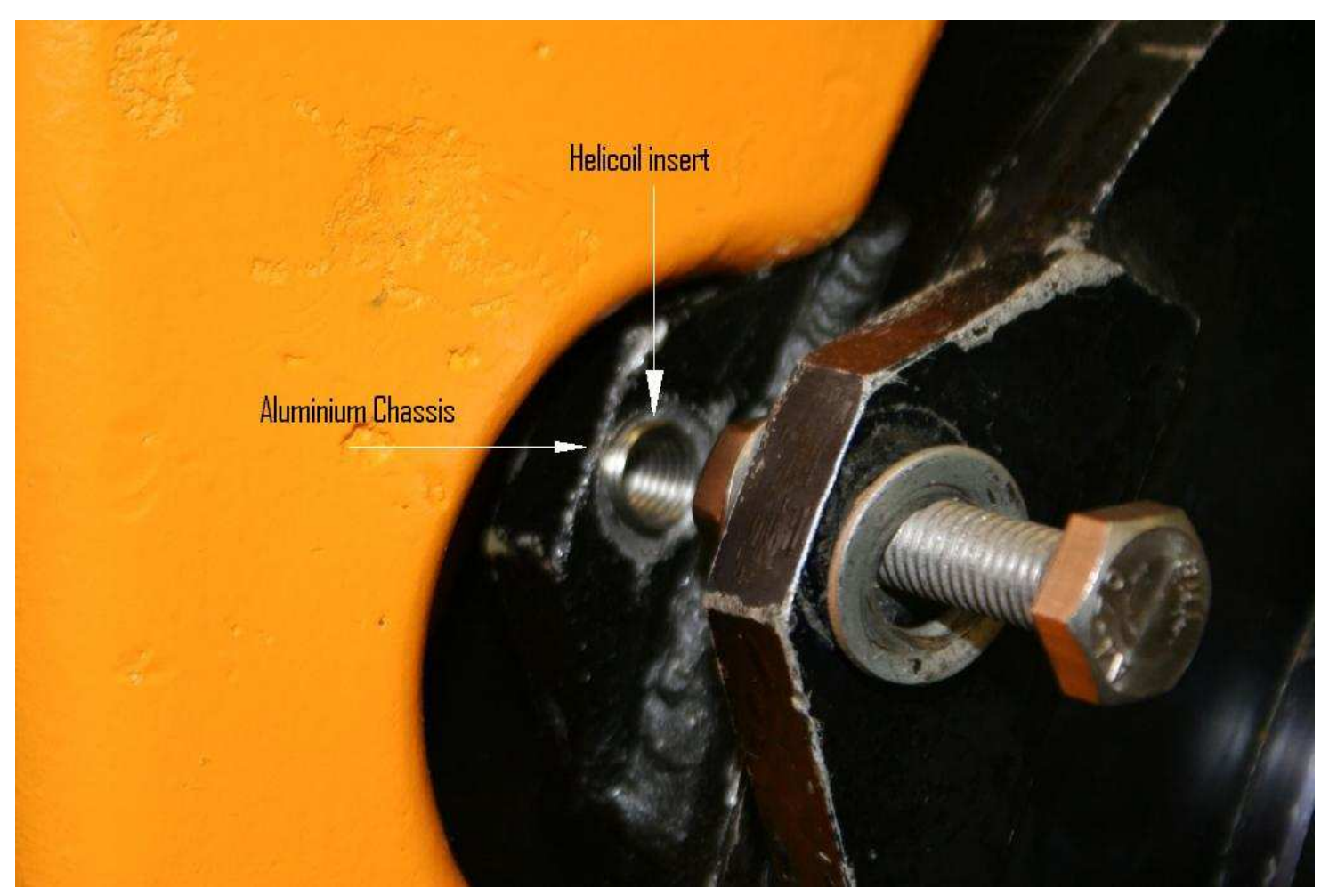

Figure 3.20 Helicoil insert and the M10 bolt 
The inserts work like a spring: when the bolts are screwed into the inserts, the inserts expand and dig into the aluminium chassis. Because the inserts are steel, the screwing and unscrewing of the bolts will not wear them away as before.

Once the bolts have been removed, the hull has to be pulled from its fitting. The method that had been used was to insert a screwdriver between the hulls and use it as a lever to open the hull. This method was not ideal because between the two hulls is the main Oring seal, which could potentially be damaged by the screwdriver. Figure 3.21 shows one of the three slots that were carefully milled into the sides of the robot hull so that screwdrivers could be inserted without damaging the O-ring.

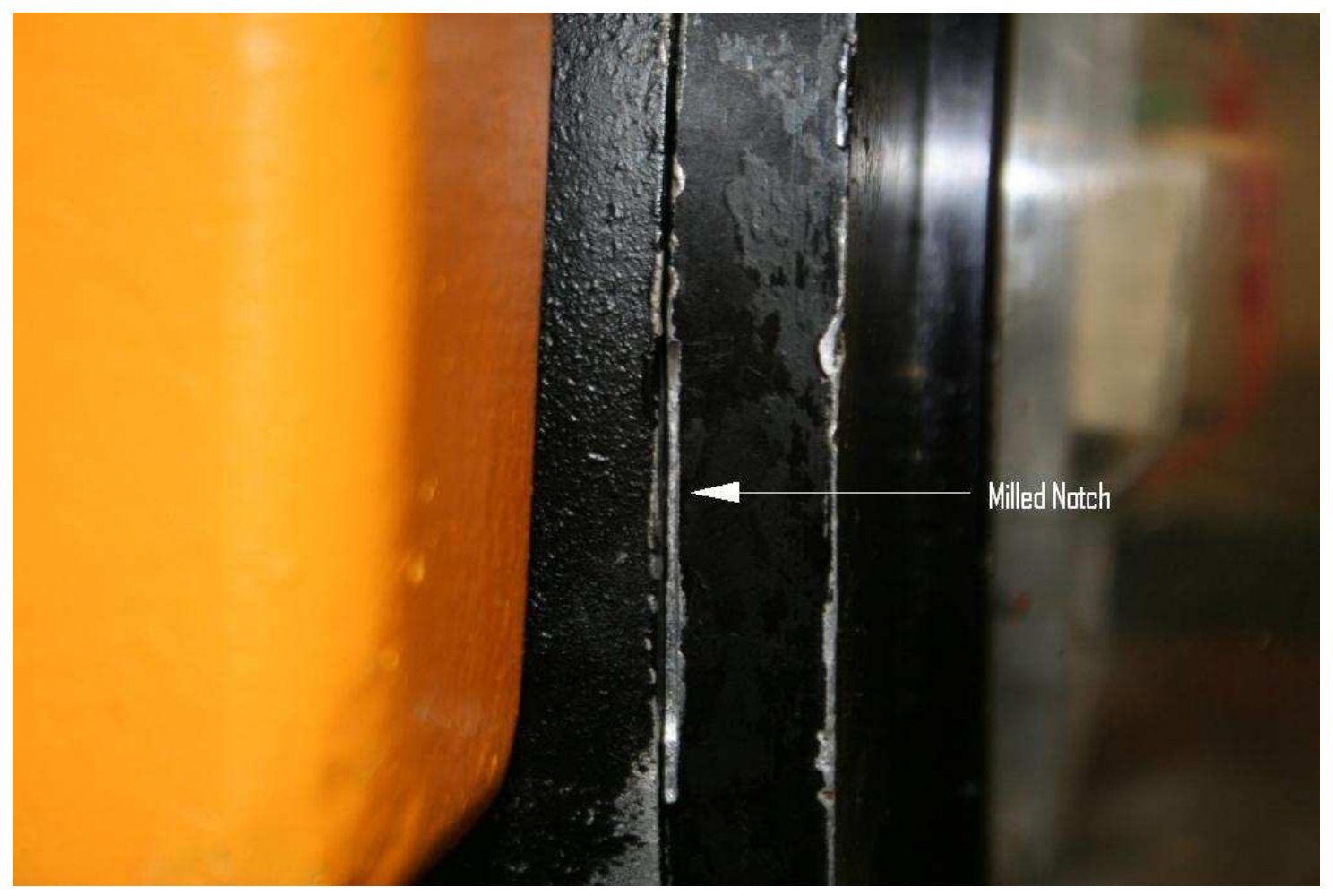

Figure 3.21 Notch for inserting screwdrivers

\subsubsection{Multiplexer Replacement}

When reading over the previous work carried out on the ROV, there were some sections of work that had very little, if any, documentation. One such section was the multiplexer 
board in the sensor compartment of the ROV. It was created to receive the multiple readings from the sensors that are placed in each of the three separate hulls. These sensors consist of temperature sensors, humidity sensors and water alarms to alert the operator of a problem or potential problem in one of the hulls.

Each hull had its own individual board, onto which the sensors and any associated electronics were mounted. The temperature sensor is a LM35 and is connected to an amplifier circuit to boost the signal. This is to measure the temperature in each of the hulls, which will show if there is either a leak (as the internal temperature may start dropping) or another problem such as shorting of wires (producing heat or fire). Also connected to the board is the HU10 humidity sensor, which can detect from 25\% to $100 \%$ humidity. The water alarm sensor is made of a $3.5 \mathrm{~mm}$ stereo plug that is wired to the sensor board. If there is no water in the hull then the plug acts as an open circuit and there is no conduction. If there is water in the hull, the water will conduct the voltage/current between the two parts and this will send a signal back to the multiplexer board. An LED is mounted on the sensor board so that a visual inspection can be made when testing the water alarm.

There is also an external temperature sensor mounted from the multiplexer board that goes through the front of the port hull, which is used to measure the external water temperature.

From the only fully populated multiplexer circuit, a schematic was reverse engineered. Because of the way the circuit was constructed it had to have all of its connections severed to remove the board. This means that if the board has to be removed for replacement the wires connecting the various boards end up shorter after reconnection, which in turn makes it harder for the inner hull to be removed from the outer hull. 


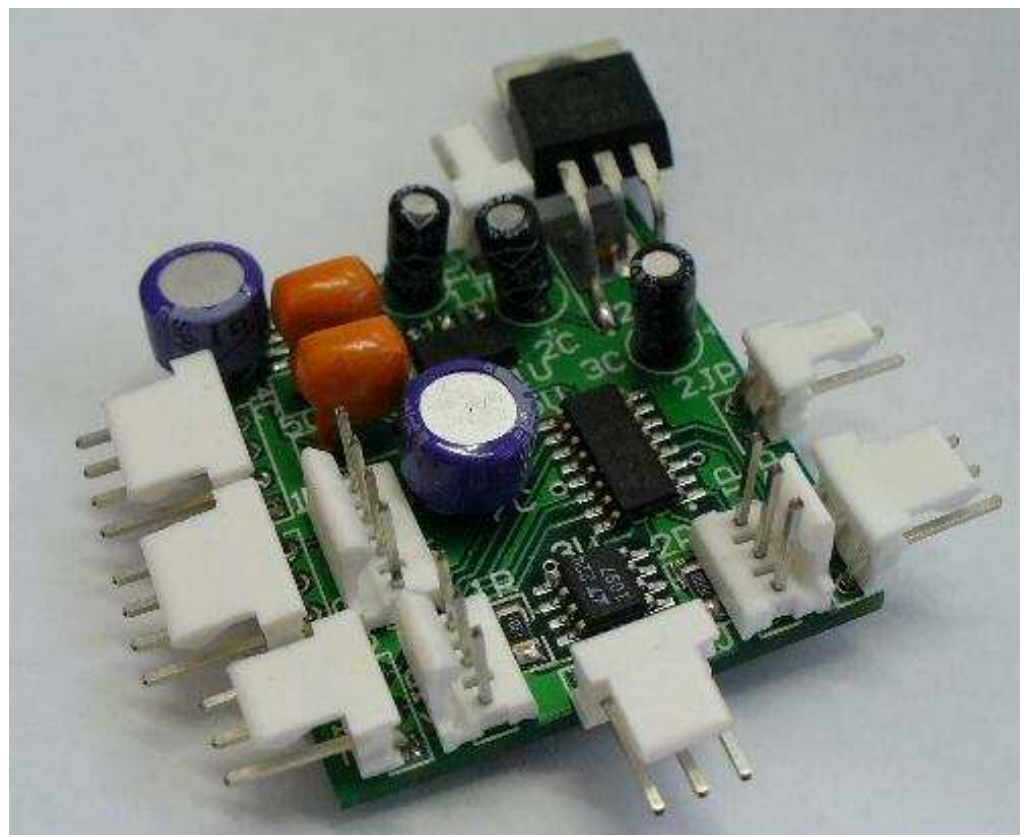

Figure 3.22 New Multiplexer board

A replacement board (Figure 3.22) was constructed to overcome these problems. This board is smaller than the original to decrease the footprint in the hull so that more electronics could be put in there at a later date. It also has connectors to allow for the removal of the circuit when servicing the port hull.

\subsubsection{Camera Bracket}

The USB web camera that is used by the operator to view underwater was stripped of all unnecessary parts including the outside casing. This would allowed the it to use the movable mount in the main hull that previously held a Panasonic 1350A video camera [22]. This left the front part of the camera with the CCD sensor and microphone held to the main circuit board by the electrical connecter, which meant that when the main hull was reassembled there was a chance the camera would fall off the connector. This was due to the weight of the front part of the lens assembly, which is made from steel, and weighs 45 grams. This is too much weight for the connecter to support. To prevent this, 
an aluminium plate (Figure 3.23) was made to hold the camera to the movable mounting bracket.

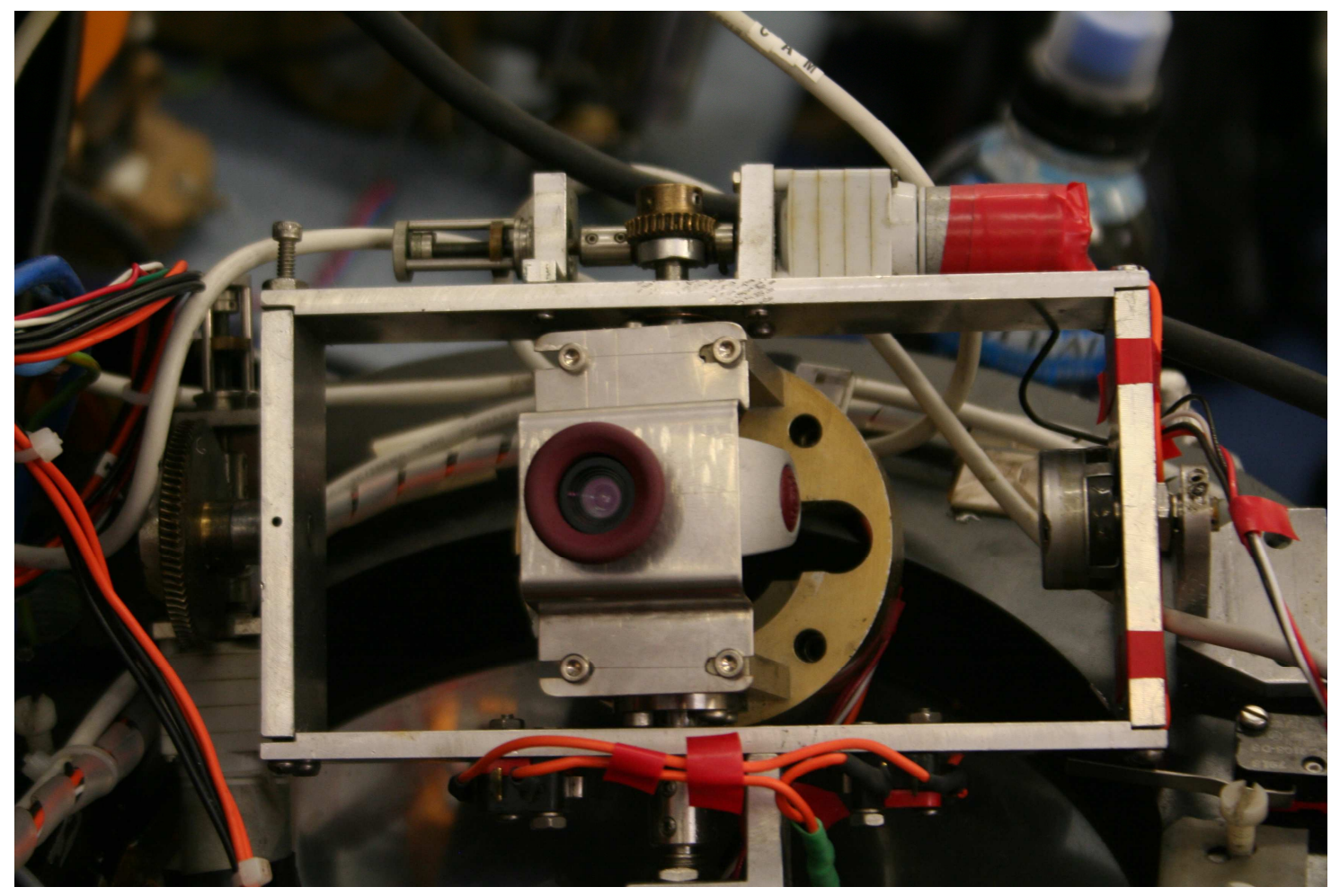

Figure 3.23 New Camera Mounting Bracket

The plate has a hole drilled in the middle to allow the lens of the camera to poke through and support the 45 gram weight of the front plate and lens assembly. This plate was then screwed into the existing camera mount using the existing screw holes, which have been retapped with M3 screws.

\subsubsection{Summary}

The design and subsequent revision of an IMU based on a ST LIS3LV02DQ accelerometer and an Analog Devices ADIS16080 gyroscope is detailed. This unit will be the main part of the INS system for the ROV. Most of the other hardware changes have been confined to the main hull. The PC, its ancillary parts, and the microcontroller 
have been replaced with new hardware and the camera, multiplexer, main chassis and cable system have had major changes to improve the ease of use.

This has not only enhanced the capabilities of the ROV's current hardware but also has increased the flexibility of the ROV and means any future upgrades do not necessarily need to change the hardware. The organizational layout of the ROV is shown in Figure 3.24 .

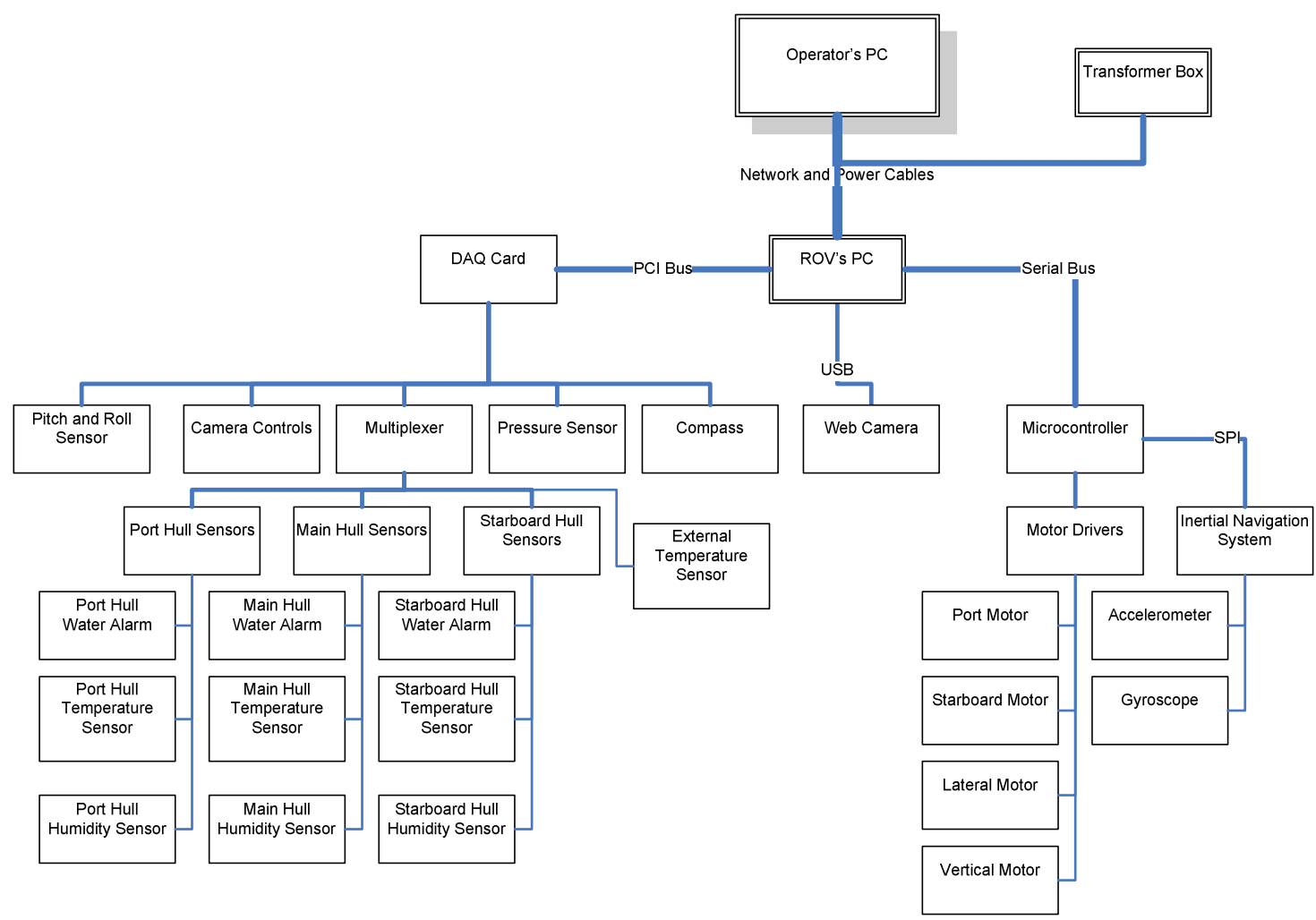

Figure 3.24 Organization Layout of the ROV and its Subsystems 


\section{CHAPTER 4. SOFTWARE}

\subsection{Types of software}

There are two distinct types of software used on the ROV: commercial and task specific. The commercial software is available for purchase from vendors and has usually been designed to efficiently handle and manage the resources and tasks that program is aiming to cover. Task specific software are programs that have not been purchased, but rather created specifically for the tasks and hardware being designed and installed in the ROV.

At the start of the project the software on the ROV was not the latest version available. It was decided that, along with the hardware, the software should be upgraded to the latest versions where possible. The operating system was considered first because it determined which other software products would be upgraded or replaced. The possible operating systems were Linux or the latest version of Windows operating system (OS). It was decided to upgrade from Microsoft's Windows 98 Second Edition to XP Professional Edition, which at the time was Microsoft's latest OS.

XP Professional Edition was chosen because of the Mechatronics Group's limited knowledge of and experience with other operating systems (such as Linux and Mac OS), and also due to the availability of software able to run on the operating system. Microsoft's XP is the most commonly used operating system in the world, and as such there are hundreds of programs compatible with it, whereas Linux and Mac OS have considerably less software available for them. In the future it may be possible for the Mechatronics Group to transition to Linux, which would solve some of the hardware issues in terms of real time communication and control of the mechatrons in the group.

The next piece of major software to be changed was the program handling the calculations from the ROV's various sensors and inputs from the user. On the original 
setup of the ROV, this program was MathWorks' Matlab Revision 6. This was upgraded to Matlab 2006b, as were the installed toolboxes required for the ROV (the Instrument Toolbox, Data Acquisition Toolbox and the Image Processing Toolbox). Also installed were the Matlab interface drivers for the IOTech DAQ card.

The program Hi-Tide from HT Soft is used to program the microcontroller. This was upgraded to the latest version, which enables the code to be emulated and tested before downloading it into the microcontroller. The downloader used is Atmel's Flexible Insystem Programmer (FLIP), which combined with the Auto ISP hardware mentioned earlier (Section 3.3.4), allows the ROV to be reprogrammed any time it is powered up including while underwater.

\subsubsection{SPI code}

The microcontroller has an inbuilt SPI interface that has to be configured to match that of the accelerometer. The timing diagrams required for communication with the accelerometer are shown in Figure 4.1.

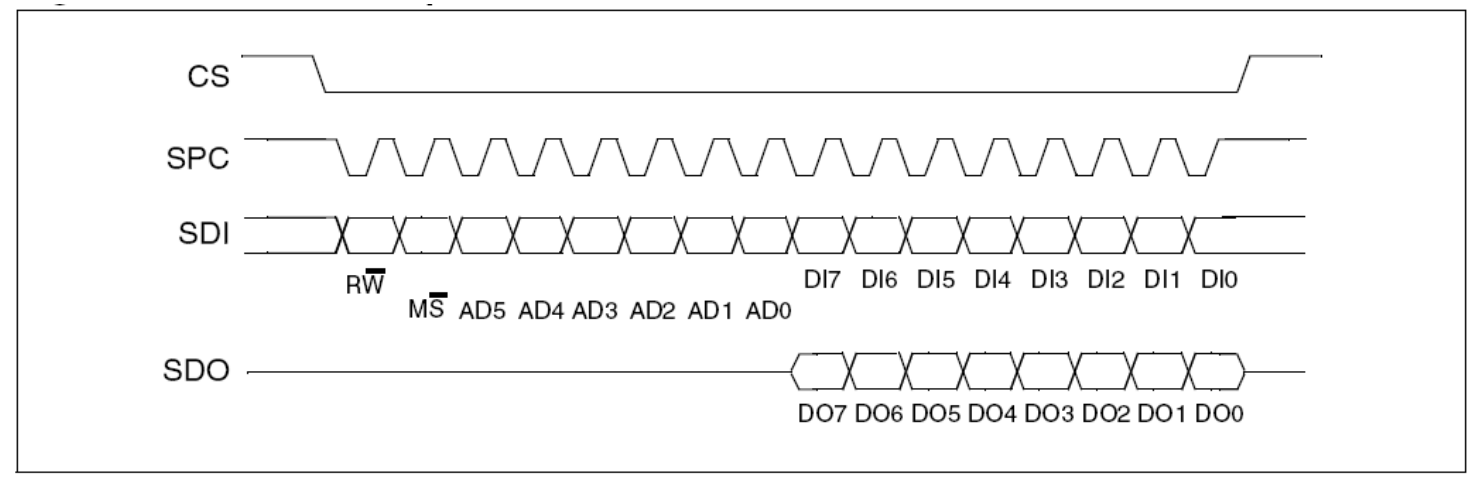

Figure 4.1 LIS3LV02DQ Timing Diagram [25]

The diagram shows that the chip select line (CS) must be set low for the chip to accept data. The data are clocked in on the falling edge of the clock signal (SCLK), and clocked out on the MISO line on the same falling edges. 
In order to allow the microcontroller to use the SPI bus, the micro must be made aware of all the registers related to the SPI bus. This required some additions to the header file at the start of the code. This header file lists whether the register can be changed (volatile) or is a fixed (non volatile) value, and its location in the microcontroller's register list. An example of each is below.

The registers added are listed in Figure 4.2. These registers are: the SPI control register (SPCON), which controls the clock rate, the clock pulse and polarity; the SPI status register (SPSCR), which controls the SPI mode flags; and the SPI data register (SPDAT), which is where all SPI data are written and read from.

static volatile unsigned char SPCON @ 0xD4;

static volatile unsigned char SPSCR \& 0xD5;

static volatile unsigned char SPDAT @ 0xD6;

\section{Figure 4.2 SPI Data Registers}

Once the registers are declared, the individual bits for each register must also be declared if that register can be addressed by bit wise operations (Figure 4.3). 


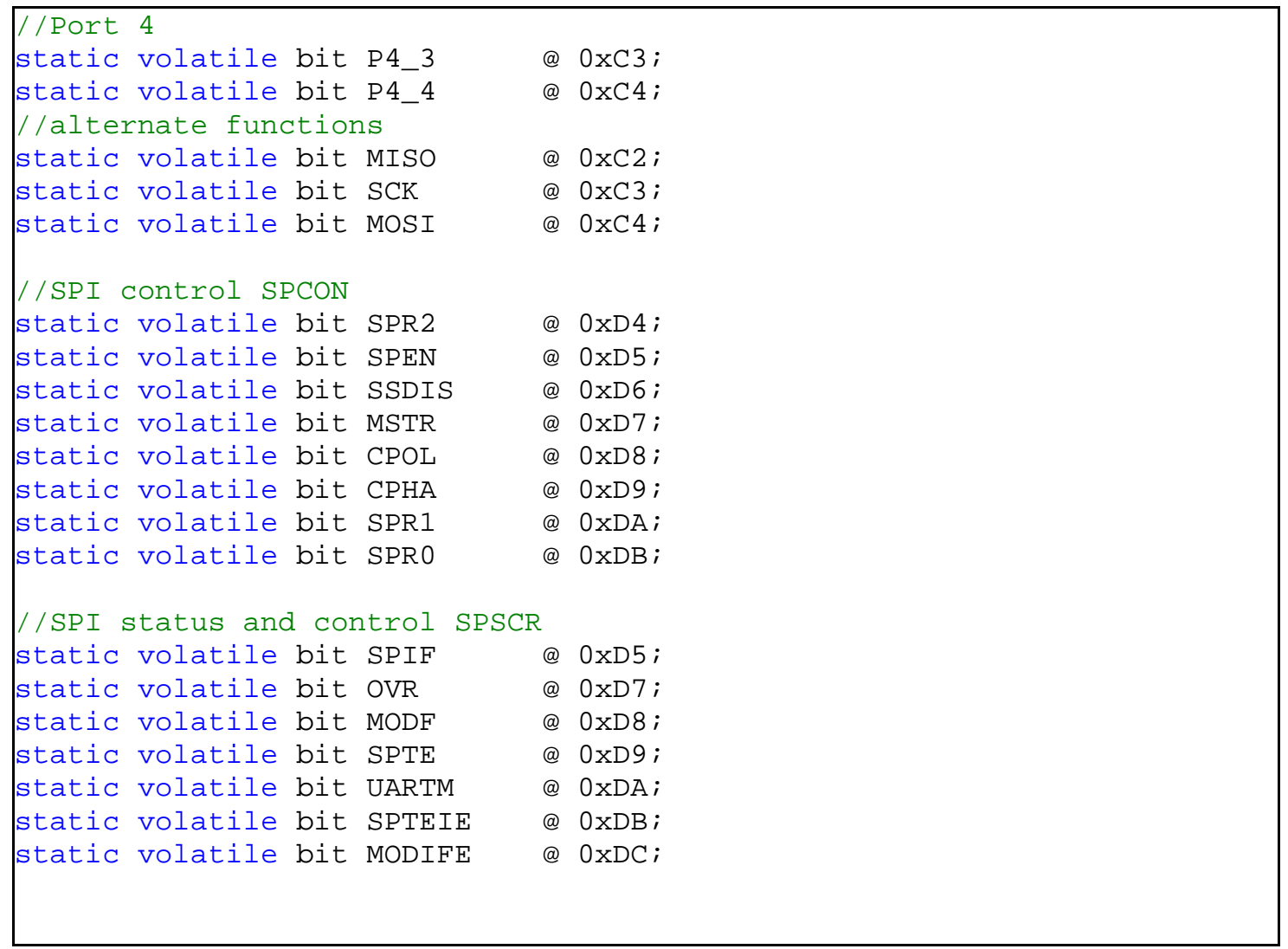

Figure 4.3 SPI Individual Bits

The code to set up the SPI on the microcontroller is shown in Figure 4.4.

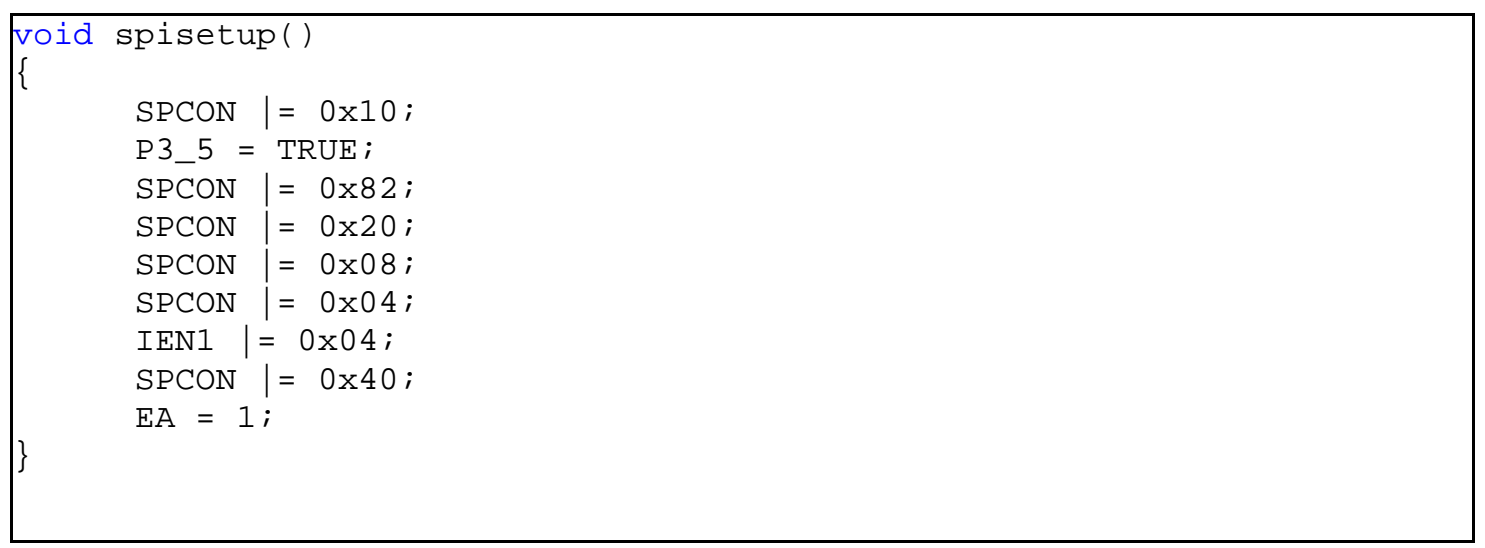


The micro is configured as the master. The master controls the timing of the SPI clock and sets the baud rate of the SPI and the signal transition edge. The CPOL register sets the transition edge to be low (as required in the timing diagram). The SPI interrupt has to be set up so as to allow the micro to receive an interrupt when a signal is received at the micro's SPI buffer. Once this is done, the SPI control is turned on to wait for data, and then all the interrupts are turned on to allow the SPI interrupt to trigger a flag in the micro.

Once configured, the SPI specific register SPDAT can be used. SPDAT handles the data that are to be transferred and received. Because SPI communication is full duplex, as the data are shifted out of the SPDAT register onto the transmit line, data coming in on the receive line can be passed straight into the register. From here it is passed to the internal data bus within the chip.

Initially the LIS3LV02DQ accelerometer is in a powered off mode: to turn it on the first control register for the device needs data transmitted to it. Reading back the device's address, which is set at the factory and is $0 \times 3 \mathrm{~A}$, will show if it is working.

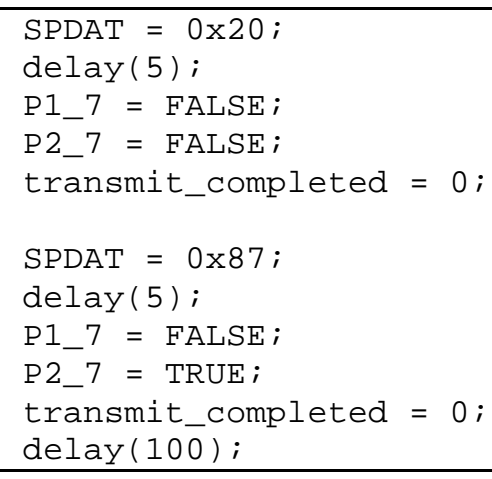

Figure 4.5 Accelerometer Transmission

The code in Figure 4.5 shows the layout for sending data to the accelerometer. The address to be written to is first put in the SPI data register. A delay is added (to allow time for the data to be transmitted) and then the transmit completed flag is reset. This flag 
is set in the SPI interrupt function. Following the address, the data that are being written are sent. An example of this is shown in Figure 4.6.

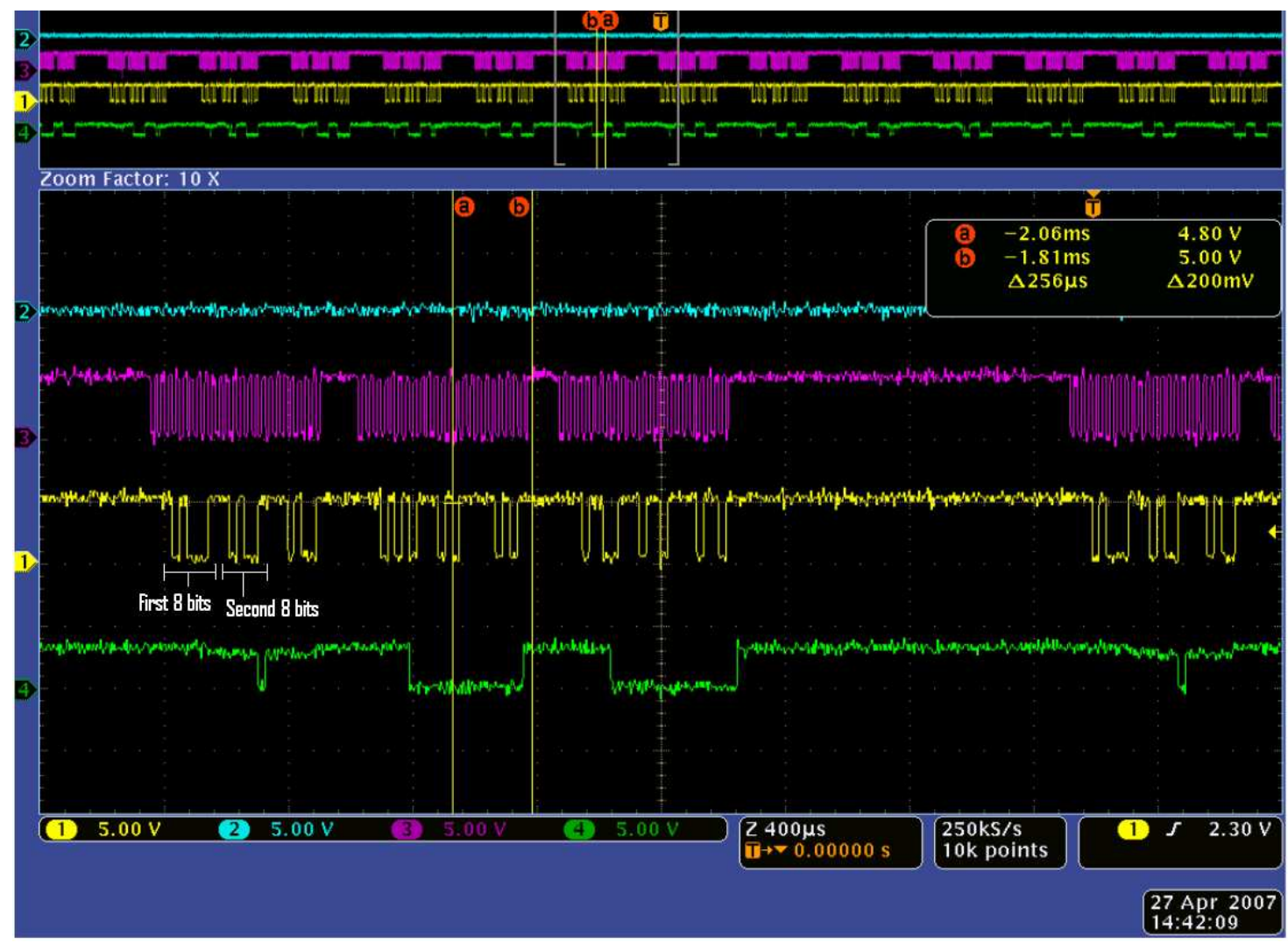

Figure 4.6 SPI Data Transmission

The three waveforms in Figure 4.6 represent the three main signal lines in SPI communication. The purple waveform is the clock signal which is generated by the microcontroller when there is data in the serial buffer. The second waveform, in yellow, is the data being sent down the SDI or MOSI line. The first 8 bits are the address for the lower register, while the second 8 bits are the address for the high register. The final waveform is the green waveform and that is the SDO or MISO line where data from the device is transmitted back to the microcontroller.

The data from the first 8 bits of the accelerometer comes back on the clock signal of the second 8 bits that are sent down the MOSI line, so the second 8 bits are repeated to 
provide a clock signal for the response from the accelerometer on the second 8 bits that were sent.

\subsubsection{Serial communications}

The calculation and conversion of the data from the accelerometer and the gyroscope is handled by the ROV's computer instead of the microcontroller because the computer has better processing capabilities (in terms of speed and processing power). The data are shifted from the micro to the computer using the serial port on both devices.

The serial communication comprises two separate parts: the first is to initialise the micro to allow it to send and receive data from the computer, and the second is to do the same on the computer.

The code for setting up the micro is as follows (Figure 4.7):

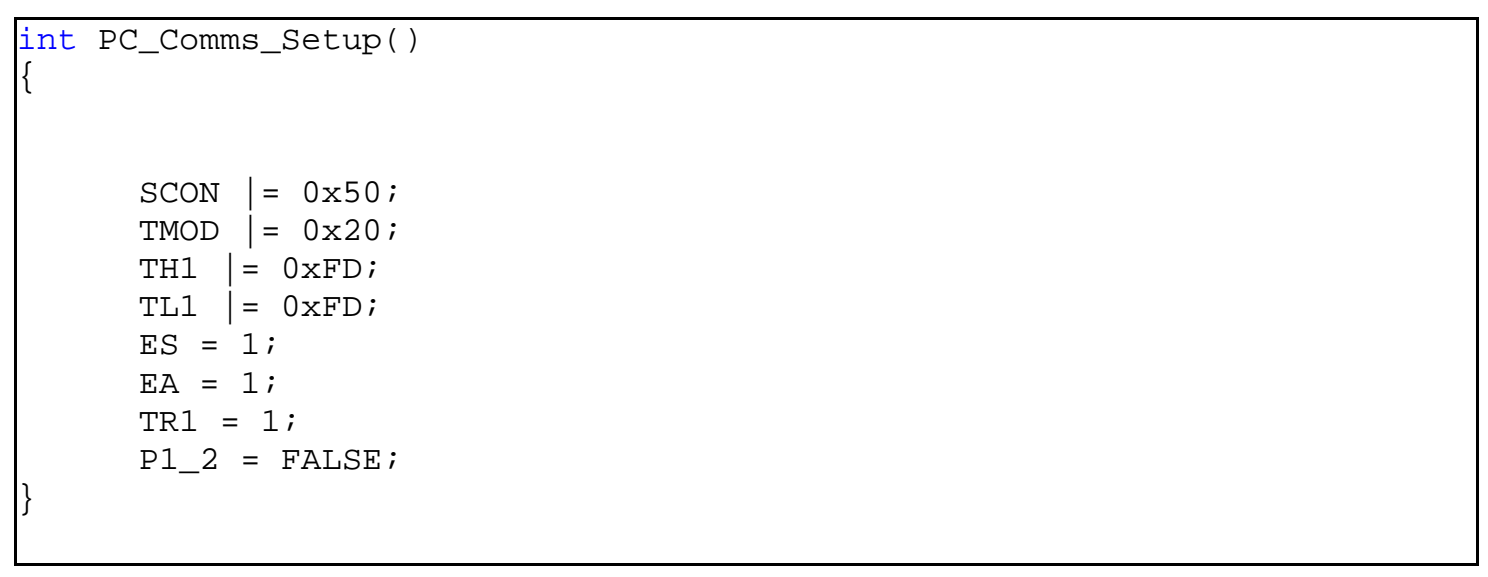

\section{Figure 4.7 Serial Communication Setup}

The SCON register contains the mode bits to select between 8 bit and 9 bit UART communications as well as the transmit and receive interrupt flags, framing error bit and the value of the $9^{\text {th }}$ bit in transmit and receive modes (if the $9^{\text {th }}$ bit is used). Setting SCON to $0 \times 50$ sets the Reception enable bit, which allows for serial communication. It also sets 
the mode of the serial data to be 8 bit data packets, with a variable baud rate to be determined. Timer 1 is used to generate the timing for the baud rate: TH1 and TL1 are the timer high and timer low registers respectively.

Once a receive flag is set the following code is run:

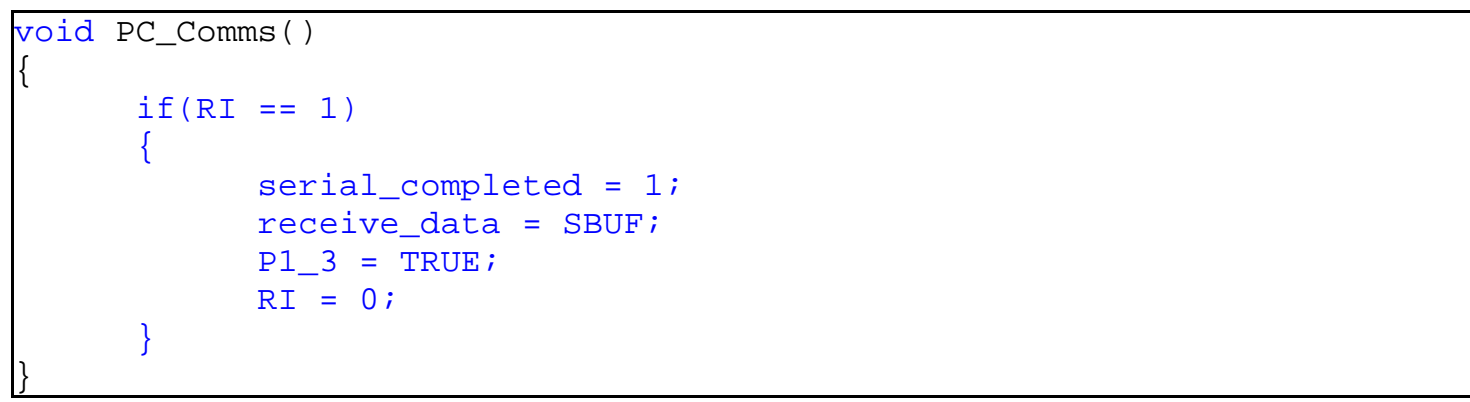

Figure 4.8 PC Interrupt code

This shifts the data from the Serial Buffer (SBUF) into the variable receive_data so that data can either be moved into, or out of the buffer and onto the transmit line. The receive flag must also be reset before leaving the if statement, so that if more data are received the micro does not lose the data.

The last part of the serial code for the micro is the code for transmitting data to the computer Figure 4.9.

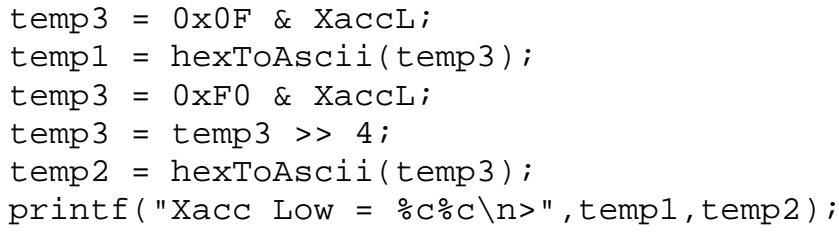

Figure 4.9 Serial Transmission code

The data from the accelerometer are stored in two parts. XaccL stores the data from the $\mathrm{X}$ direction's lower 8 bits and XaccH stores the upper 8 bits. Each variable is then separated into parts so that it can be converted from hexadecimal. XaccL is ANDed with 0x0F to 
get the lower part. This is passed to the hexToAscii function, part of which is shown in Figure 4.10.

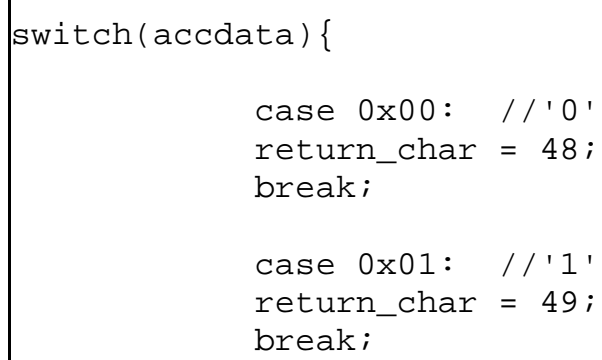

Figure 4.10 hexToAscii code

The switch statement tests the variable accdata, which is the data that have been passed to the function. When it matches one of the cases the code is flagged as true and the line of code immediately after the case is executed. Once this is done the program exits the function. If the variable does not match any of the case statements it outputs the default code, which has been setup to return the character J. Once the lower bits of the XaccL variable are converted to ascii, $\mathrm{XaccL}$ is ANDed with $0 \mathrm{xF}$ to get the higher bits of the variable (Figure 4.10). The higher bits are then shifted four places to the right, to where the lower bits used to be. The bits in the lower position are then passed to the hexToAscii function for conversion. The printf command transmits the data along the serial lines into the computer so Matlab can read them.

On the PC side of the serial port the Matlab programming language handles all the serial communications. This makes it easier to manipulate the results because the same program gets the results, handles all the calculations to control the robot, and maps the vehicle's position.

For Matlab to use the serial port it first needs to be initialised. Figure 4.11 shows the code which sets up the communication port the microcontroller is connected to, the baud rate, 
and other parameters as shown above. The serial port must then be opened in Matlab to allow the transmission and reception of data.

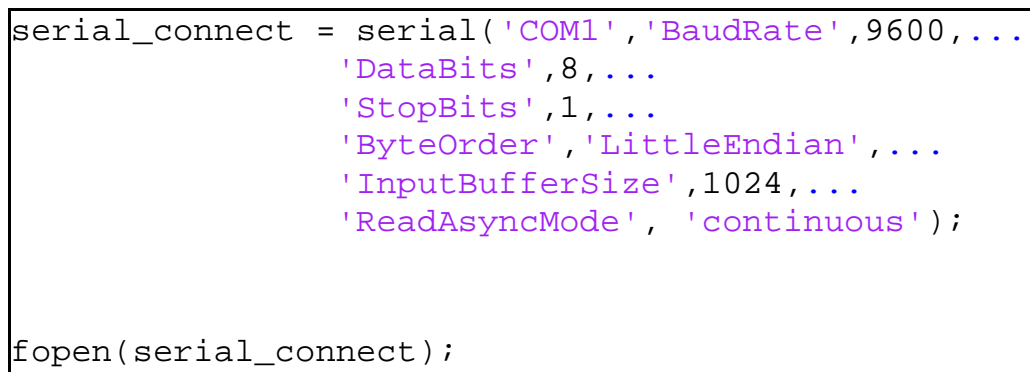

Figure 4.11 Matlab Serial Connect

Once all the data have been taken from the serial lines, and communication with the micro is discontinued, the function serial_disconnect is called. This function closes the file that the data are being stored into, before deleting and clearing serial connect from the workspace Figure 4.12.

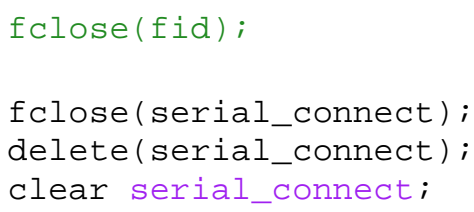

Figure 4.12 Closing and Deleting the File

\subsubsection{Data Storage and Conversion}

The serial store function opens a file to store the data and checks to see if there are any data on the serial lines. 
fid $=$

fopen (strcat ('C: \Program Files \MATLAB \R2006b\work \Accelerometer-

', strcat (datestr (clock, 30)), '.txt'), 'a+');

fidd $=$

fopen (strcat('C: \Program Files \MATLAB \R2006b\work \Accelerometer in dec-

', strcat (datestr (clock, 30)), '.txt'), 'a' ) ;

fprintf (fid, datestr (now));

fprintf (fid, '\n');

fprintf (fid, '\n');

fprintf (fidd, datestr(now));

fprintf (fidd, '\n');

fprintf (fidd, ' $\backslash \mathrm{n}^{\prime}$ ) ;

Figure 4.13 Creating the files

It first creates two files, one named Accelerometer, and another named Accelerometer in dec. The time and date are concatenated to the ends of each of the file names, allowing the exact file to be found at a later date. The current date and time are also written at the start of the file. The file name or the directory location can be changed just by changing the name in the first line.

Once the files are set up the program checks the number of bytes available at the serial port. The program can then take the number of bytes available from the serial port and store them in the array data. 
The data are transferred into an array (val) and converted from a number string into hexadecimal with the Matlab function num2hex (Figure 4.14). The array val has been pre-allocated at the start of the function so that it uses memory more efficiently with the values of 40000 rows and 9 columns.

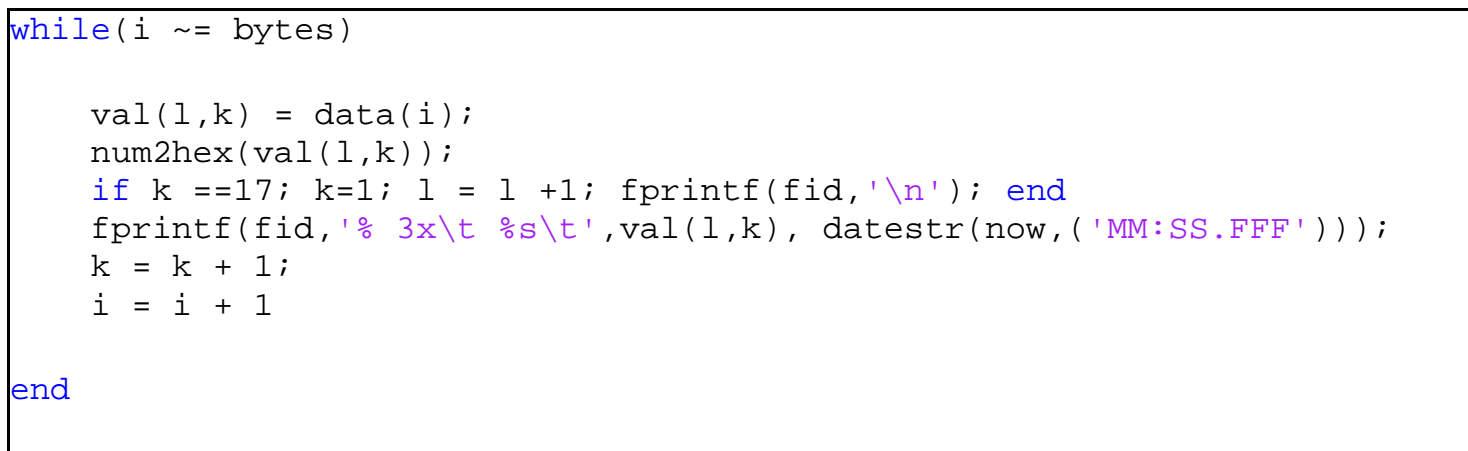

Figure 4.14 Updating the file

The data are stored in 16 columns in the text file, with the current time stored behind it to create a time stamp that will be used as the time in the integration of the data. The time stamp is based on the time the data reached the serial port, not when the data were created. However, the time taken to transfer the data from the IMU to the serial port is faster than the smallest fraction of time that Matlab can measure, so it was decided that the time it enters the serial port is the best time available. The datestr(now(MM:SS.FFF)) function outputs the date and time based on the point in time inside the brackets. Now takes the time from the system clock. The only relevant parts from the time are the minutes (MM), seconds (SS) and milliseconds (FFF). From Figure 4.15 we can see the data in hexadecimal and then the time in the MM:SS.FFF format. 


\begin{tabular}{|rlrlrlrl|}
\hline 32 & $08: 13.550$ & 30 & $08: 13.566$ & $\mathrm{~d}$ & $08: 13.566$ & $\mathrm{~d}$ & $08: 13.582$ \\
$\mathrm{a}$ & $08: 13.597$ & 30 & $08: 13.613$ & 30 & $08: 13.613$ & $\mathrm{~d}$ & $08: 13.629$ \\
$\mathrm{~d}$ & $08: 13.644$ & $\mathrm{a}$ & $08: 13.660$ & 36 & $08: 13.675$ & 31 & $08: 13.691$ \\
$\mathrm{~d}$ & $08: 13.707$ & $\mathrm{~d}$ & $08: 13.722$ & $\mathrm{a}$ & $08: 13.754$ & 37 & $08: 13.769$ \\
0 & $08: 13.800$ & $\mathrm{~d}$ & $08: 13.800$ & $\mathrm{~d}$ & $08: 13.800$ & $\mathrm{a}$ & $08: 13.816$ \\
30 & $08: 13.816$ & 31 & $08: 13.832$ & $\mathrm{~d}$ & $08: 13.832$ & $\mathrm{~d}$ & $08: 13.847$ \\
$\mathrm{a}$ & $08: 13.847$ & 37 & $08: 13.863$ & 46 & $08: 13.863$ & $\mathrm{~d}$ & $08: 13.863$ \\
$\mathrm{~d}$ & $08: 13.879$ & $\mathrm{a}$ & $08: 13.879$ & 30 & $08: 13.894$ & 30 & $08: 13.894$ \\
0 & $08: 13.910$ & 30 & $08: 13.910$ & $\mathrm{~d}$ & $08: 13.925$ & $\mathrm{~d}$ & $08: 13.925$ \\
$\mathrm{a}$ & $08: 13.941$ & 61 & $08: 13.941$ & $3 \mathrm{e}$ & $08: 13.941$ & $\mathrm{~d}$ & $08: 13.957$ \\
$\mathrm{~d}$ & $08: 13.957$ & $\mathrm{a}$ & $08: 13.972$ & 30 & $08: 13.972$ & 34 & $08: 13.988$ \\
$\mathrm{~d}$ & $08: 13.988$ & $\mathrm{~d}$ & $08: 13.988$ & $\mathrm{a}$ & $08: 14.004$ & 30 & $08: 14.004$ \\
0 & $08: 14.019$ & $\mathrm{~d}$ & $08: 14.019$ & $\mathrm{~d}$ & $08: 14.035$ & $\mathrm{a}$ & $08: 14.035$ \\
30 & $08: 14.050$ & 30 & $08: 14.050$ & $\mathrm{~d}$ & $08: 14.066$ & $\mathrm{~d}$ & $08: 14.066$ \\
$\mathrm{a}$ & $08: 14.066$ & 37 & $08: 14.082$ & 43 & $08: 14.082$ & $\mathrm{~d}$ & $08: 14.097$ \\
$\mathrm{~d}$ & $08: 14.097$ & $\mathrm{a}$ & $08: 14.113$ & 34 & $08: 14.113$ & 30 & $08: 14.113$ \\
0 & $08: 14.129$ & $\mathrm{~d}$ & $08: 14.129$ & $\mathrm{a}$ & $08: 14.144$ & 37 & $08: 14.144$ \\
46 & $08: 14.160$ & $\mathrm{~d}$ & $08: 14.160$ & $\mathrm{~d}$ & $08: 14.160$ & $\mathrm{a}$ & $08: 14.175$ \\
30 & $08: 14.175$ & 30 & $08: 14.191$ & 30 & $08: 14.191$ & 30 & $08: 14.207$ \\
& & & & & & & \\
\hline
\end{tabular}

\section{Figure 4.15 Fragment of the Data from the Text File}

These data consist of five main parts. The letter ' $a$ ', from the above text file, is the line feed character. This signifies the start of the transmission of the data. After this are the two bytes of data. These are made up from the lower and higher parts for each individual direction, with two bytes for the lower parts and two bytes for the higher parts. There are two special cases, however, that also occur after the line feed character. The first case is the characters represented by 61 as seen in the tenth line of text in the file above. This character represents the letter ' $a$ ' in the ASCII format. These are added to the code and the file for two reasons:

The first reason is for debugging. Once the microcontroller and the IMU are running, a hyper terminal window may be opened to view the data coming into the computer through the serial port. When the info is displayed in the hyper terminal window, as shown in Figure 4.16, the letter ' $\mathrm{a}$ ' will appear before the start of the $\mathrm{X}$ direction information. This allows a check of the output. 


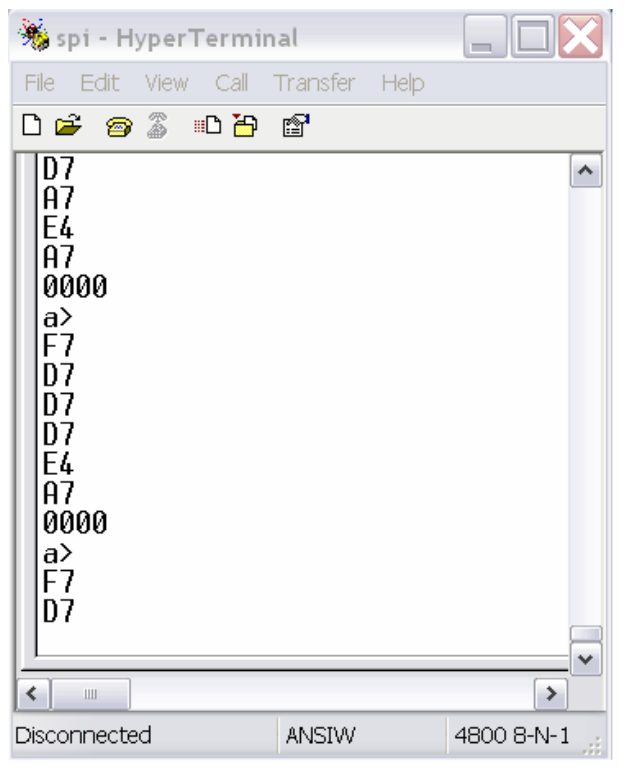

Figure 4.16 Hyper terminal Debugging

The second reason is for reading the raw data in the file. Having the character ' $a$ ' stored in the file allows someone to look over the file at a later date and find where the start of each successive transmission is. This allows for data matching using other data gathered from sources on the ROV or external vehicles at a later stage, as well as being able to replot the path that the ROV took.

The second special case is the character represented by the ' $3 \mathrm{e}$ ' from Figure 4.16. This character is the greater than sign (>). This is also a mark, and is used in another program run by Matlab to pick up the start of the file so that the individual parts of the directions can be used to produce the acceleration. The accelerometer is set up to send a 12 bit signal back to the computer, but the microcontroller requires a 16 bit data message. The highest 4 bits in the upper acceleration direction register are arbitrarily assigned by the accelerometer the same value as the $11^{\text {th }}$ bit of the upper direction register for the purposes of data transmission. 


\begin{tabular}{|l|l|l|l|l|l|l|l|}
\hline XD15 & XD14 & XD13 & XD12 & XD11 & XD10 & XD9 & XD8 \\
\hline
\end{tabular}

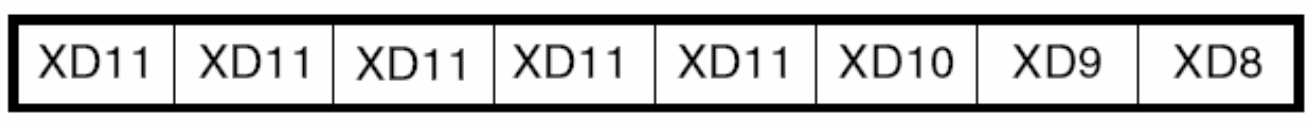

Figure 4.1716 bit (Top) and 12 bit (Bottom) data values for the $\mathrm{X}$ direction acceleration

Figure 4.17 shows the difference between setting the accelerometer to transmit a 12 bit data message and a 16 bit data message.

The letter ' $d$ ' in the text file (Figure 4.15) is the carriage return character while the letter ' $a$ ' is the line feed character. The carriage return character is called twice and signifies the end of each line or data transmission from the serial port. The line feed character moves the cursor to the next line for the next set of data.

Once the data have been stored, they are converted to hexadecimal and then sorted based on the information that is required. The first grouping or 'cases' are data that are not required: thus is ignored. The second group is for the character $3 \mathrm{E}$. When this character is detected in the sequence the count variable is reset. This variable keeps track of the particular part in the sequence that the code is at. 


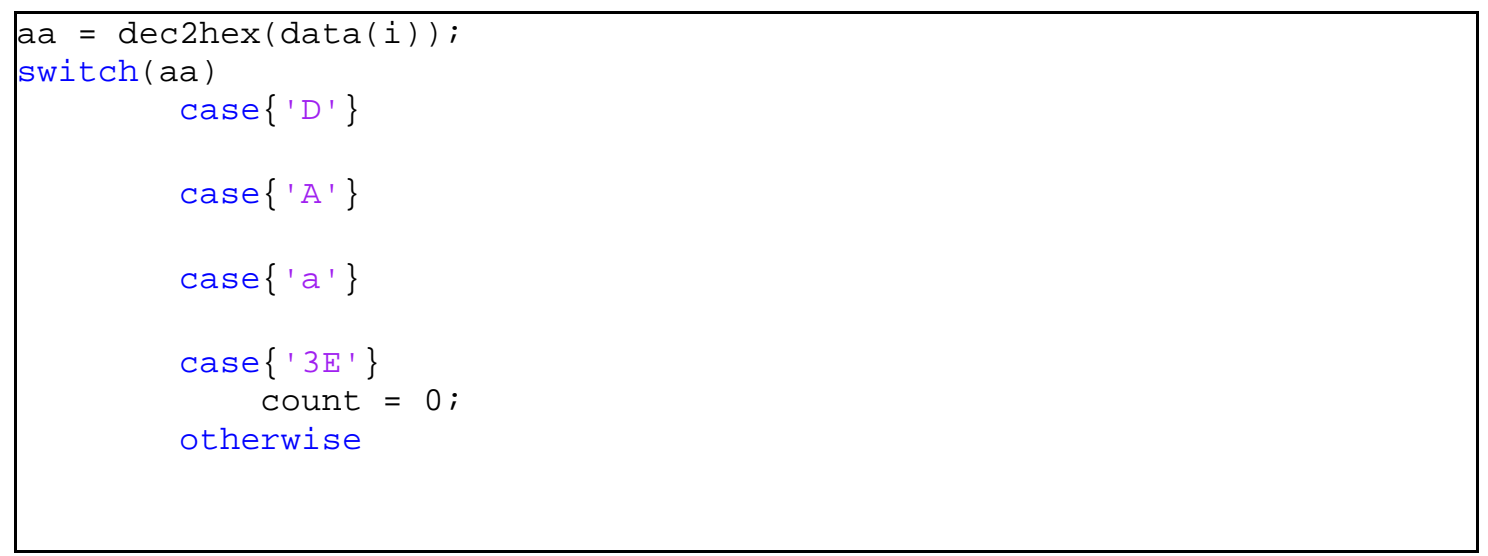

Figure 4.18 Sequence Switch Statement

If neither of those cases is triggered, then the data are sorted based on the variable count. This sorts the data into the variables $X I 1, X I 2, X h 1$ and $X h 2$, which represent the $X$ direction lower bits $(\mathrm{X} 11, \mathrm{X} 12)$ and the $\mathrm{X}$ direction higher bits (Xh1, Xh2). It then converts the data from hexadecimal into decimal and stores them in these variables, as shown in Figure 4.20. The $\mathrm{Y}$ direction and $\mathrm{Z}$ direction are also set up as per Figure 4.19, with the only difference being the value against which the count is tested.

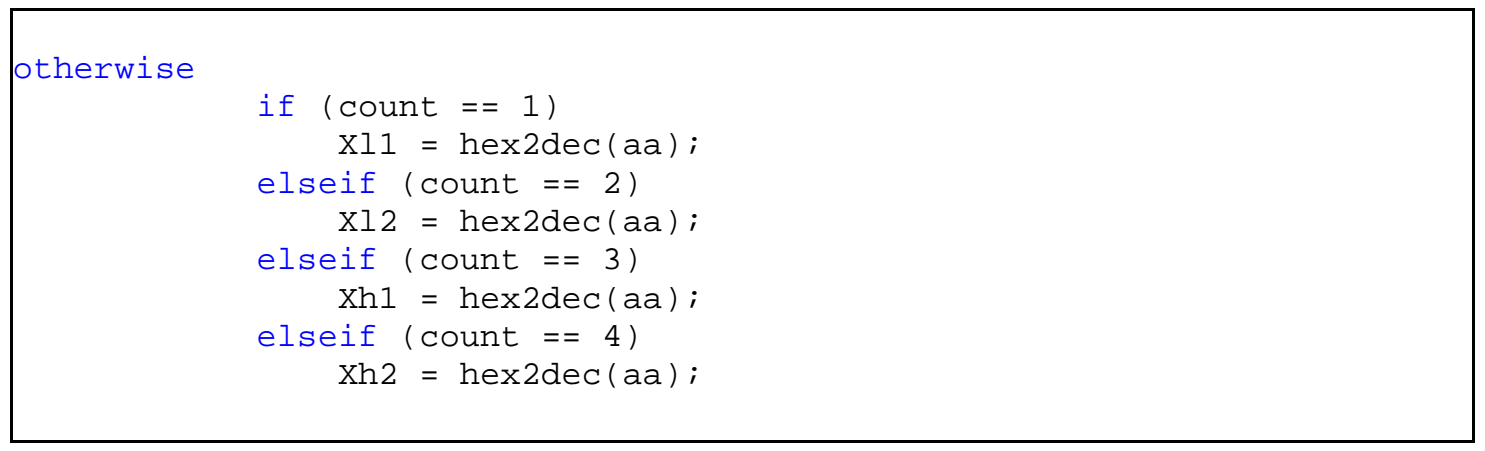

Figure 4.19 Data Conversion into Hexadecimal

The 12 bits required are then assembled by concatenating the first three variables together and storing them into a temporary variable. This is converted into decimal and then stored in the second file created Accelerometer in dec and also time stamped. 


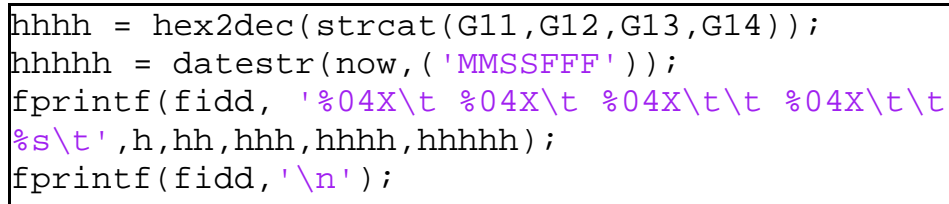

Figure 4.20 Writing the data and time stamp to the file

The 04X|t fragments in the above code, Figure 4.20, set out the formatting of the data. The 0 tells the program to pad the data with zeros and the 4 sets out the data width to be four characters wide. Hexadecimal representation is shown by the $X$ and the following $\backslash t$ tabs the write position in the file across to the right.

This creates a data file that contains information as shown in Figure 4.21.

\begin{tabular}{|lllll|}
\hline 201 & 403 & 605 & $7 A 5 E$ & 4317218 \\
201 & 403 & 605 & $7 A 5 E$ & 4319453 \\
201 & 403 & 605 & $7 A 5 E$ & 4320984 \\
201 & 403 & 605 & $7 A 5 E$ & 4321015 \\
201 & 403 & 605 & $7 A 5 E$ & 4321031 \\
201 & 403 & 605 & $7 A 5 E$ & 4322984 \\
201 & 403 & 605 & $7 A 5 E$ & 4325234 \\
201 & 403 & 605 & $7 A 5 E$ & 4326125 \\
201 & 403 & 605 & $7 A 5 E$ & 4326156 \\
201 & 403 & 605 & $7 A 5 E$ & 4326171 \\
\hline
\end{tabular}

Figure 4.21 Data in the Hexadecimal Format

\subsubsection{Mapping from One Frame of Reference to Another}

To correctly calculate the ROV's position once the data from the accelerometer and the gyroscopes have been transmitted, the data have to be transformed into the operator's frame of reference. To do this some matrix manipulation is used, with four different matrices multiplied to the data from the ROV: one matrix for the accelerometer data and one each for the three different gyroscope readings. 


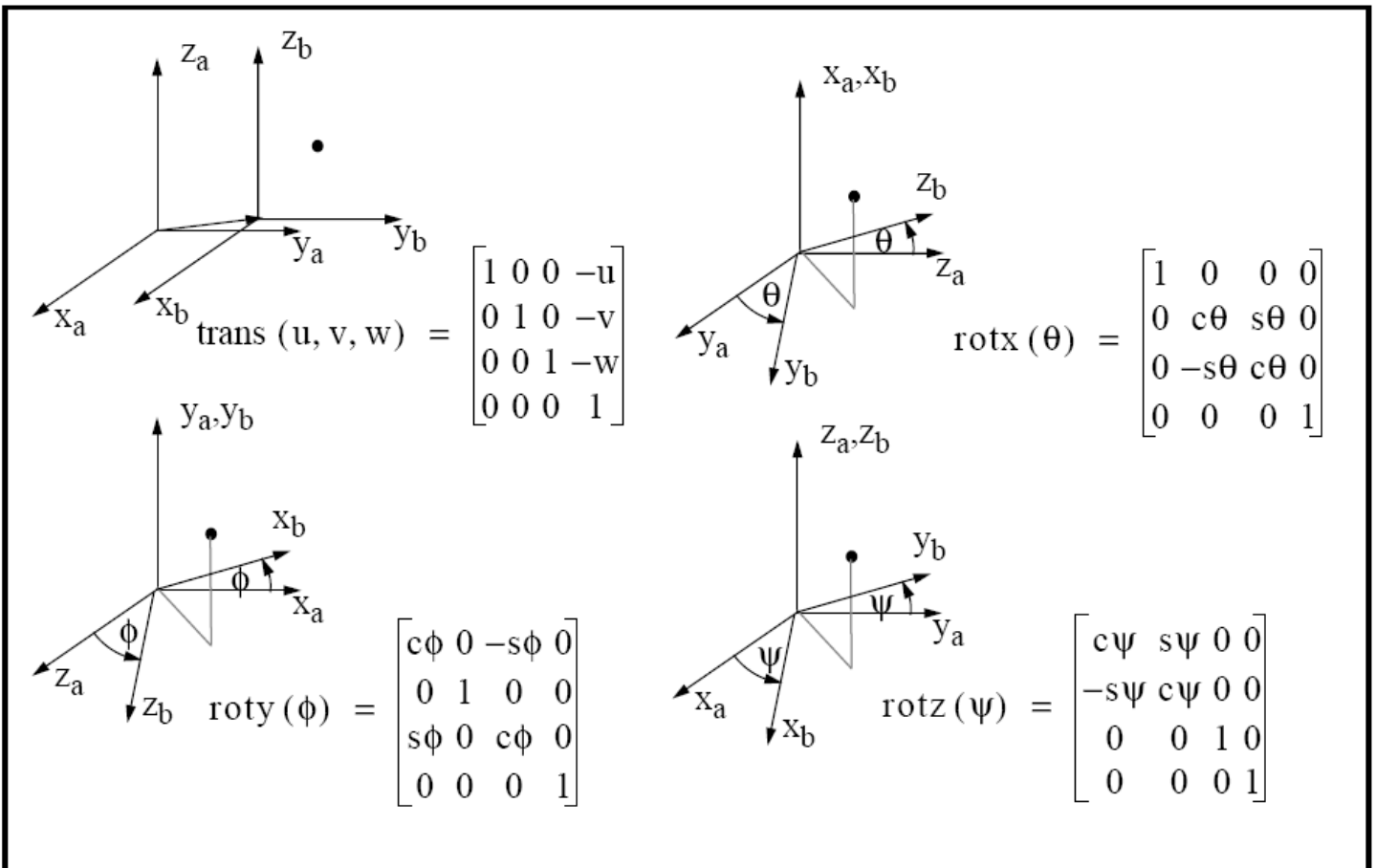

Figure 4.22 Frame of Reference Manipulation Matrices [26]

In Figure 4.22, $s$ and $c$ stand for sine and cosine respectively. This manipulation is coded in Matlab. Figure 4.23 shows the total matrix when the four matrices are multiplied together [22].

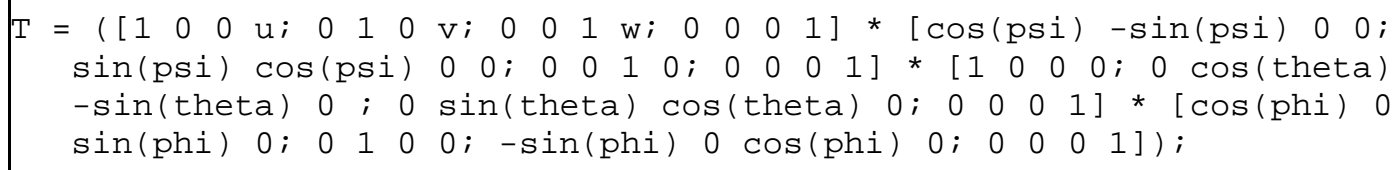

Figure 4.23 Matlab Frame of Reference matrix

Since the data from the accelerometer are expressed in terms of acceleration, they must first be changed into a distance. To do this, the acceleration must be integrated twice with respect to time. 
Before the data are converted from acceleration into distance, however, the data from the micro have to be changed from two's complement (which is what the accelerometer outputs) into the SI units of metres per second per second. This is accomplished with the following code:

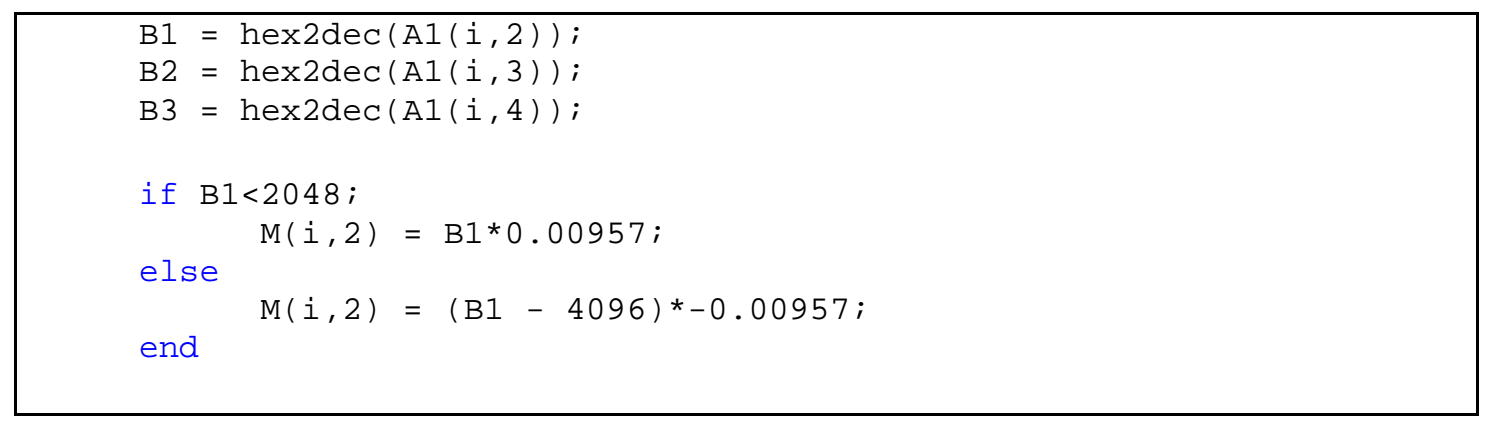

Figure 4.24 Two's Complement Conversion

The data for $\mathrm{X}, \mathrm{Y}$ and $\mathrm{Z}$ direction are stored in array $\mathrm{A} 1$, before the hex2dec function is called (Figure 4.24). This converts the hexadecimal value in A1 into a decimal value, which is then stored in the variables B1, B2 and B3 for the $\mathrm{X}, \mathrm{Y}$ and $\mathrm{Z}$ directions respectively. These are then compared in a if statement to 2048, which is the value for 2 $\mathrm{g}$ of acceleration (see Table 5.1). If the value being compared is less than 2048 it means the acceleration is a positive acceleration and it is multiplied by $0.00957 \mathrm{~ms}^{-2}$ which is calculated from dividing $9.82 \mathrm{~ms}^{-2}$ by 1024 bits. If the value is greater than 2048 , then 4096 is subtracted from it before being multiplied by $-0.00957 \mathrm{~ms}^{-2}$. This is then stored into the array $M$.

In Matlab there is an inbuilt function for integration. Initially there are no reference values for the integration so an array of zeros has to be created into which the data are loaded.

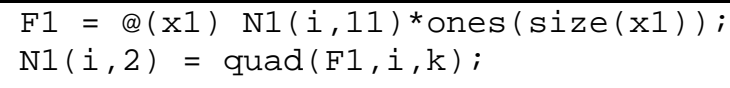


The quad function in Figure 4.25, is the command to integrate. The three variables used to describe the integral are F1 (the initial array of zeros with the data in it), $i$ (the current position in the array), and $\mathrm{k}$ (the next position in the array).

The function to integrate from velocity to distance, Figure 4.26, is similar to Figure 4.25, except that since the array has already been created, the ones(size(x1)) part of the equation is not needed.

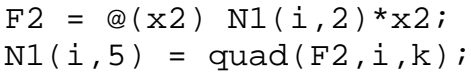

Figure 4.26 Integrating to get Distance from Velocity

The variables $\mathbf{i}$ and $k$ are the same value as previously used in the integration. Once the distance has been calculated and stored in an array, the results from the gyros are also stored into the same array as the distance, velocity and acceleration. From here the results are put into the frame of reference matrix, which will give the ROV's position. The results are shown on three separate moving graphs: one for each direction.

\subsubsection{Graphical User Interface}

Originally the Graphical User Interface (GUI) was written in National Instruments LabVIEW (a high level programming language). This was changed to Matlab by a group of Austrian students who worked on the ROV as part of their 3-month work project [27].

The first figure, Figure 4.27 shows the GUI that was originally developed for the ROV by Jennifer King [21]. This GUI had all the basic functions including the motor driver controls, depth, and the camera window. 


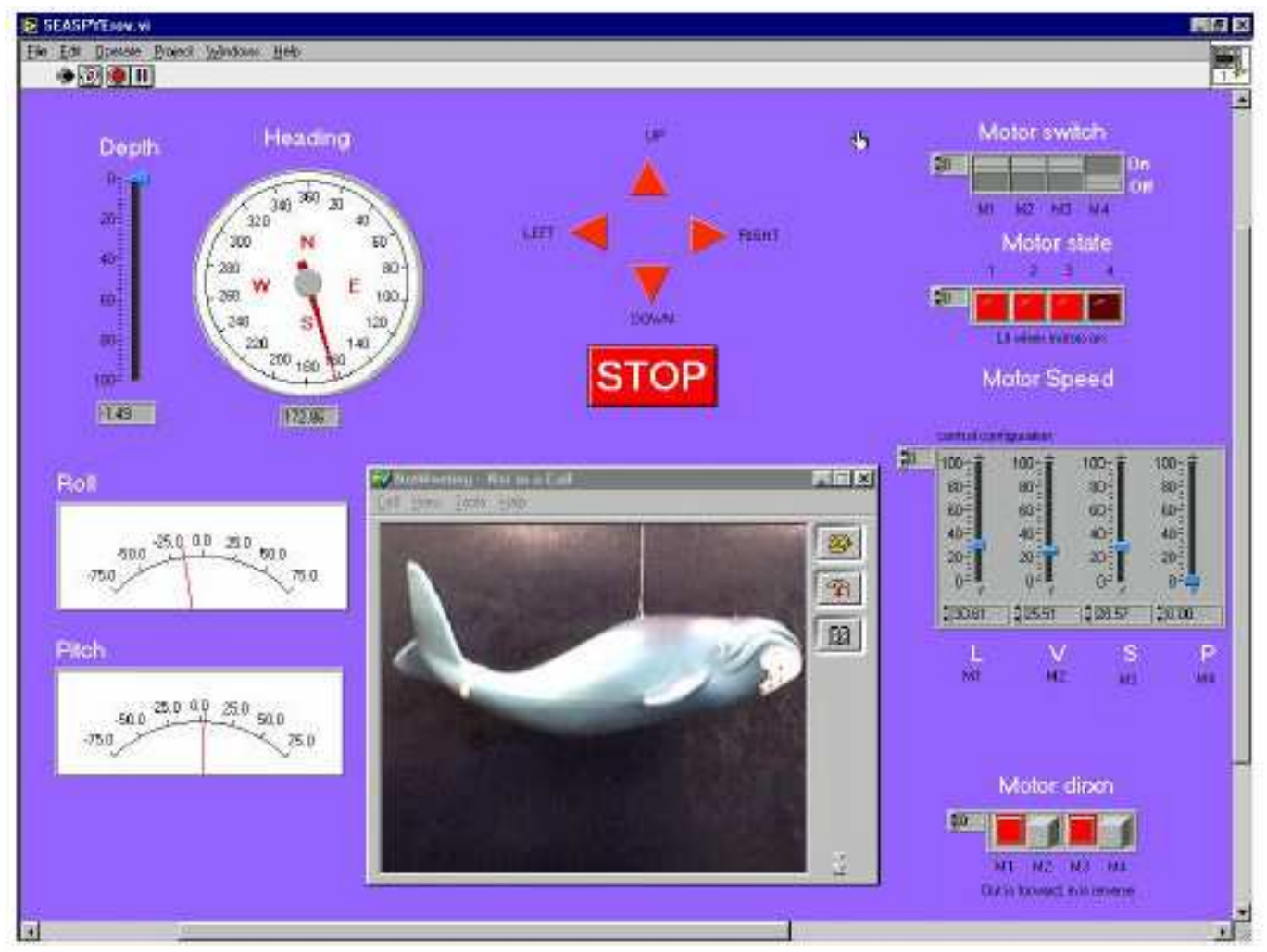

Figure 4.27 Original GUI

When this was changed to Matlab, the new sensor displays were also included in the GUI. Although this is a tidy GUI it is not an ideal layout for control. To investigate an effective GUI, commercial systems were investigated by the author. A good practical example of a GUI is that found in the cockpit of an aircraft: each individual display can be viewed easily, and there is usually a specific pattern to the layout. 


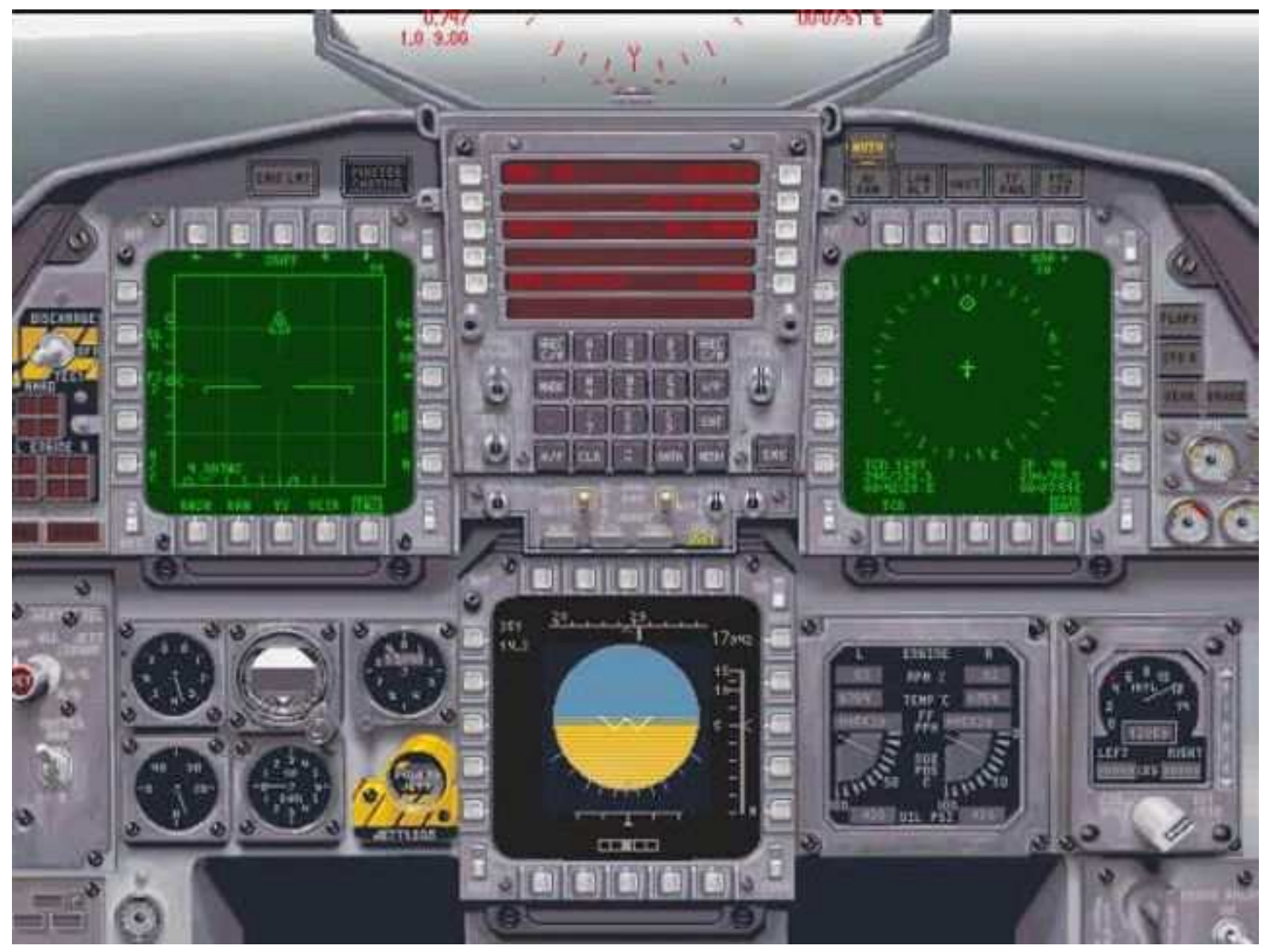

Figure 4.28 F15 Cockpit Layout

As can been seen in Figure 4.28, the position and size of instruments helps organize the incoming data for the pilot. With the above layout, a quick scan of the displays can give the pilot all the required information without having to spend time interpreting the data.

Combining data from multiply sensors onto one display frees up more space on the operators control panel and if the sensors relate to each other it helps the operator get a better feel for the controls. The display that can do this is the artificial horizon, which is the large screen at the bottom of the picture in Figure 4.29. It is made up of a pitch ladder that shows how steep the angle of attack of the vehicle is, a roll rate ladder to show the extent and direction it is rolling, and a yaw ladder that shows the twisting about the centre of the robot. 


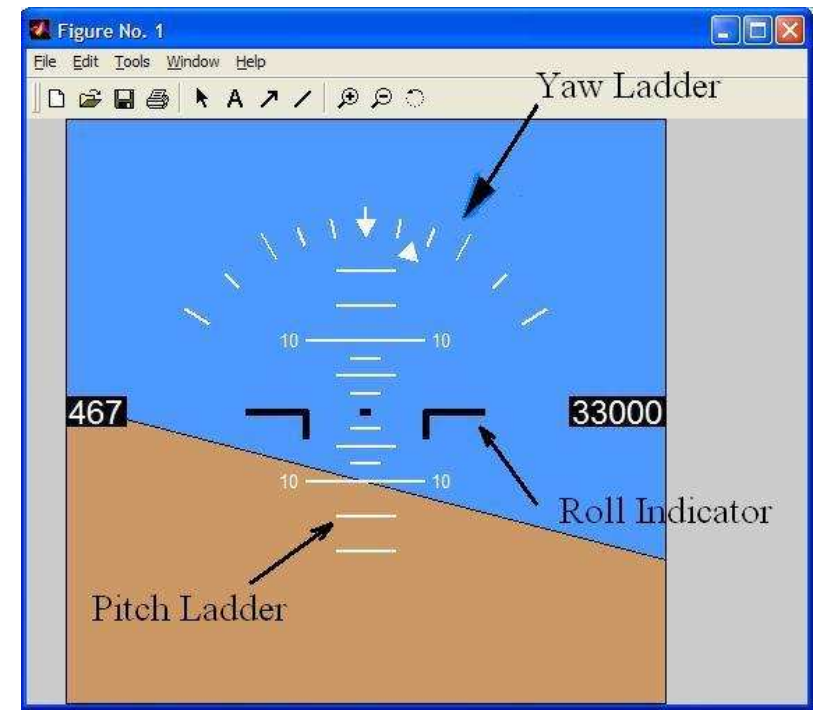

Figure 4.29 Labelled Artificial Horizon

Combining those three displays into one makes it easier to visualise what the vehicle's motion is at any point in time, as well as clearing up space on the GUI. The Artificial Horizon was placed in the top left part of the GUI and was quite large as shown in Figure 4.30. This helps the operator view it while simultaneously controlling the ROV and monitoring the rest of the controls and displays. Below this on the left hand side is the compass. The data for the compass display come from the Wesmar DC700 Flux-gate compass, which is mounted at the front of the port hull.

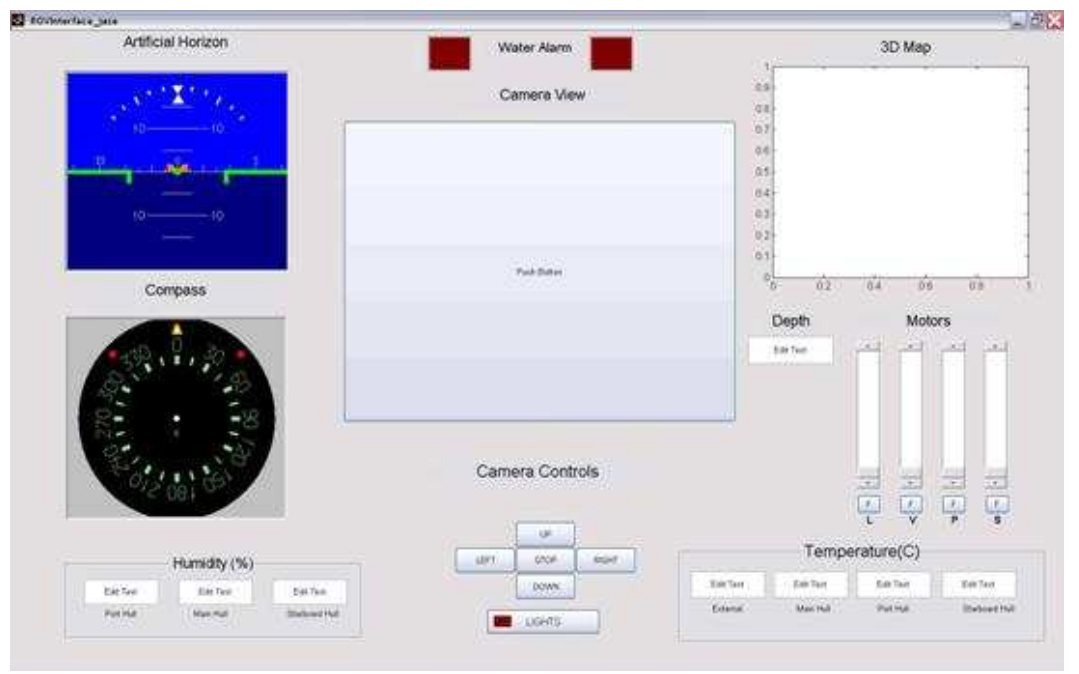

Figure 4.30 New GUI Layout and Design 
At the bottom left of the GUI the humidity sensor readings are grouped together. These are text boxes that display the calibrated value measured by the sensor. To the right of this are the buttons for camera control and the underwater lights. The camera control buttons control the servos in the main hull that drive the webcam frame. These buttons can also be controlled by the joysticks hat switch if the joystick is set up. In the light button there is a light that shows whether the underwater lights are on.

To the right of the light button at the bottom of the right side of the GUI is the temperature display box. It is set up like the humidity area of the GUI, and all the temperatures are displayed as a numerical value. These values are read from the multiplexer board through the DAQ card that is installed in the ROV.

In the middle of the right hand side of the GUI, above the temperature display, are the motor control sliders and direction buttons. There is a slider for each of the four separate motors: port, starboard, lateral and vertical. When the direction button is toggled the letter on the button changes from $\mathrm{F}$ (forwards) to B (backwards). The sliders themselves can be set using the number keys to set the value that the motors are to be on for, or with a mouse. To the left of the motor control is the depth display, which provides a digital description of the actual depth.

In the middle of the GUI is the display for the camera's view. Because the main use of this vehicle is for exploration of underwater objects, the camera's window should be the operator's main focus therefore it needs to be in the middle of the screen and quite large. This is fed directly from the webcam in the main hull of the ROV. The maximum resolution of the camera is $640 \times 480$, so it fits nicely in the GUI. Just above the top of the camera window are the water alarm lights, which turn on whenever any of the water sensors are set activated. This lets the operator know that there is water in the hulls, so that the ROV can be shut down to avoid further damage to the electronics. Because the vehicle has a slightly positive buoyancy, when shut down the vehicle will slowly float towards the surface allowing for salvage and repairs. 


\section{CHAPTER 5. RESULTS}

\subsection{Testing}

A number of tests were carried to out test the IMU and the code. The tests are a stationary tests through a set of measured areas. In the case of moving tests the results are from three different cases, namely:

- Accelerometer only measurements

- Gyroscope only measurements

\subsubsection{Stationary Test}

The stationary test involves setting up the IMU, primarily the accelerometer on a desktop surface, and gathering data over a 5 minute period. Each direction is tested individually with $\mathrm{a}+1 \mathrm{~g}$ and $\mathrm{a}-1 \mathrm{~g}$ acceleration range. This gives 3 sets of data to compare against. A 5 minute period is used because it is the same period used to create a reference point when the ROV is to be used in an operational setting. 


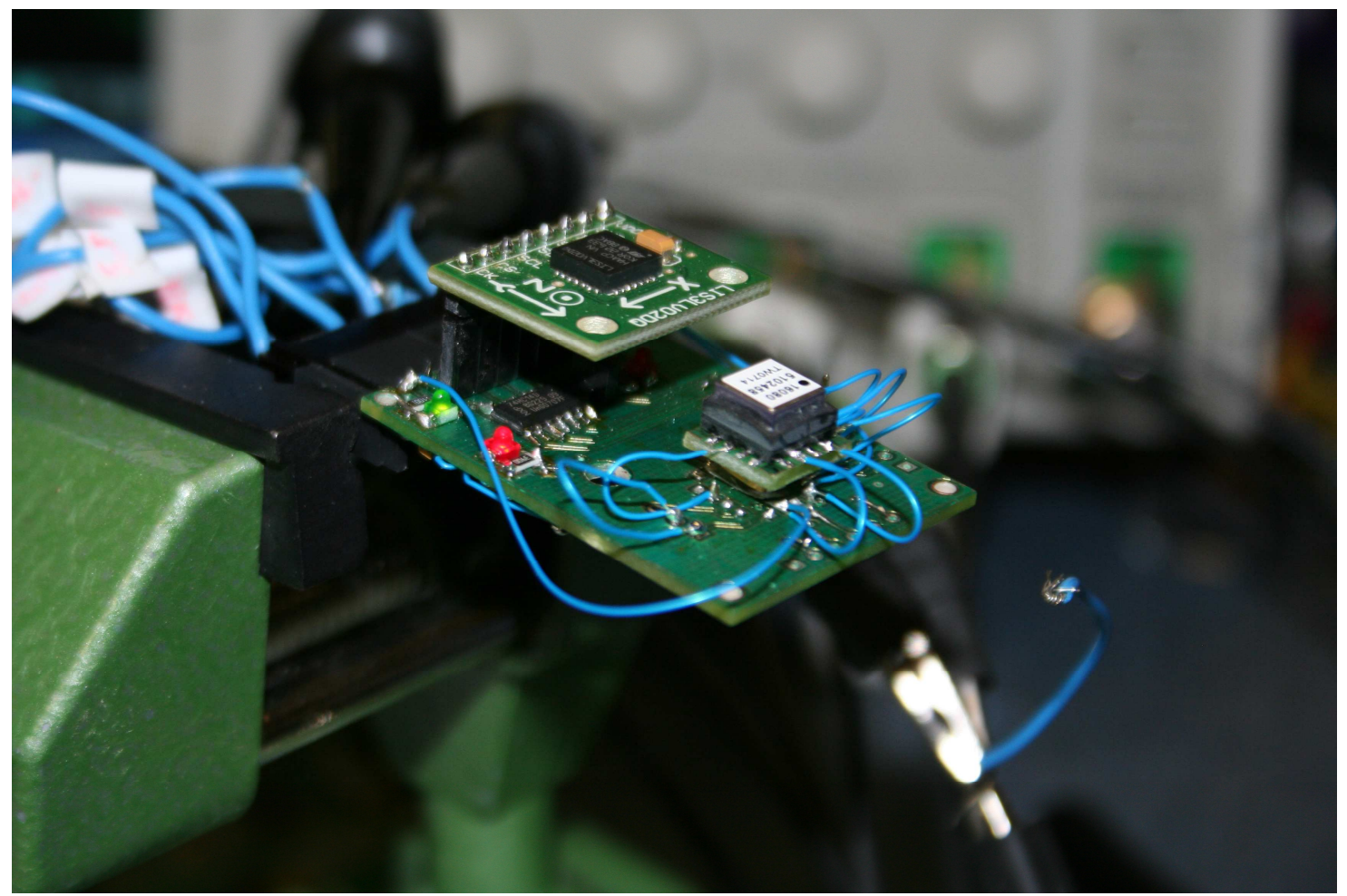

Figure 5.1 Static Test Platform for Testing

Figure 5.1 shows the static testing setup. A vice holds the IMU board by the connector to the micro, with the accelerometer placed towards the connector at the back of the board and the gyroscope on a slightly raised standoff at the front. The standoff board is used due to the problems encountered when trying to solder the gyroscope onto the IMU board. The standoff board has slightly larger pads to increase the likelihood of getting a good contact with the pads on the underside of the gyroscope. The vice has a ball joint between the top and base which allows the vice head to be tilted to test the accelerometer in all directions.

The data are initially stored as a hexadecimal value, with a don't care byte in the left most position, which is set to be zero and three bytes of data. This ensures that when converted to decimal the leading byte doesn't affect the result. To change from two's complement, the data are compared to the value of 2048 , which is the value for $2 \mathrm{~g}$ of acceleration from the accelerometer. If the datum point is greater than this, then 4096 is subtracted to convert the point into a negative value. The value 4096 is calculated from the 
accelerometer datasheet which states that for every g of acceleration there is a change in output of $1024 \mathrm{LSb}$. For the $2 \mathrm{~g}$ setting that we have selected the maximum range is from $-2 \mathrm{~g}$ to $+2 \mathrm{~g}$, which gives a total acceleration range of $4 \mathrm{~g}$. An output of $1024 \mathrm{LSb}$ per $\mathrm{g}$ at a total acceleration range of $4 \mathrm{~g}$ gives the value 4096 .

Table 5.1 shows the expected output from the accelerometer at a full scale of $2 \mathrm{~g}$, with the data configured for Little Endian mode, where the lower bytes are in the lower direction register, in this case register OUTX_L(28H), and being set in 12 bit right justified mode.

\begin{tabular}{|l|l|l|}
\hline Acceleration Values & Register 28h & Register 29h \\
\hline $0 \mathrm{~g}$ & $00 \mathrm{~h}$ & $00 \mathrm{~h}$ \\
\hline $350 \mathrm{mg}$ & $66 \mathrm{~h}$ & $01 \mathrm{~h}$ \\
\hline $1 \mathrm{~g}$ & $00 \mathrm{~h}$ & $04 \mathrm{~h}$ \\
\hline$-350 \mathrm{mg}$ & $9 \mathrm{Ah}$ & FEh \\
\hline$-1 \mathrm{~g}$ & $00 \mathrm{~h}$ & FCh \\
\hline
\end{tabular}

Table 5.1 Example of Acceleration Data in 12 bit Right Justified Mode [25]

The first four bits in all of the transmissions are exact copies of the $11^{\text {th }}$ bit of transmitted data because the accelerometer is set up in 12 bit right justified mode. If the accelerometer was set up in 16 bit left justified mode then the table would look like Table 5.2 .

\begin{tabular}{|l|l|l|}
\hline \multicolumn{1}{|c|}{ Accelerometer Values } & Register 28h & Register 29h \\
\hline $0 \mathrm{~g}$ & $0 \mathrm{xh}$ & $00 \mathrm{~h}$ \\
\hline $350 \mathrm{mg}$ & $6 \mathrm{xh}$ & $16 \mathrm{~h}$ \\
\hline $1 \mathrm{~g}$ & $0 \mathrm{xh}$ & $40 \mathrm{~h}$ \\
\hline$-350 \mathrm{mg}$ & Axh & E9h \\
\hline$-1 \mathrm{~g}$ & $0 \mathrm{xh}$ & C0h \\
\hline
\end{tabular}

Table 5.2 Example of Acceleration Data in 16 bit Left Justified Mode [25] 
The x's in Table 5.2 are 'don't care' states. This is because possible noise in the system may swamp the $\mathrm{x}$ positions, making any data they hold unreliable in some environments.

Once the data have been changed from two's complement, they are multiplied by 0.00957 , which is calculated by dividing gravity $\left(9.82 \mathrm{~ms}^{-2}\right.$ or $\left.1 \mathrm{~g}\right)$ by the $1024 \mathrm{LSb} / \mathrm{g}$. This gives a value for each single bit of the data. From the data sheet, the non linearity on the $\mathrm{X}$ and $\mathrm{Y}$ axes is $+/-2 \%$ and for the $\mathrm{Z}$ axis it is $+/-3 \%$ of the full scale, (which in the ROV's case is 2 g's).

This means that for a $1 \mathrm{~g}$ acceleration, the resulting datum value should be $9.82+/-$ $0.3928 \mathrm{~ms}^{-2}$ for acceleration in $\mathrm{X}$ and $\mathrm{Y}$ directions and $9.82+/-0.5892 \mathrm{~ms}^{-2}$ for acceleration in the $\mathrm{Z}$ direction. The following figures, Figures $5.2-5.7$ show the acceleration over a 30 second window for $+/-1 \mathrm{~g}$ in all three axis. The median values for all three axis are shown in Table 5.3.

\begin{tabular}{|l|l|l|}
\hline \multicolumn{1}{|c|}{ Axis } & + Acceleration & - Acceleration \\
\hline $\mathbf{X}$ & 9.7039 & -10.1155 \\
\hline $\mathbf{Y}$ & 9.7901 & -9.8092 \\
\hline $\mathbf{Z}$ & 10.2494 & -9.4264 \\
\hline
\end{tabular}

Table 5.3 Median Acceleration Values

These values fall with in the specifications of the data sheet and show that the device is operating correctly.

Figure 5.2 shows the graph produced when the positive $\mathrm{X}$ is actuated due to gravity. The values range from $12 \mathrm{~ms}^{-2}$ to $8 \mathrm{~ms}^{-2}$. The non-actuated directions, $\mathrm{Y}$ and $\mathrm{Z}$, show a range from $-2 \mathrm{~ms}^{-2}$ to $2 \mathrm{~ms}^{-2}$. The median for the $\mathrm{Y}$ and $\mathrm{Z}$ directions are $-0.172 \mathrm{~ms}^{-2}$ and 0.498 $\mathrm{ms}^{-2}$ respectively, which is within the tolerances of the device. 


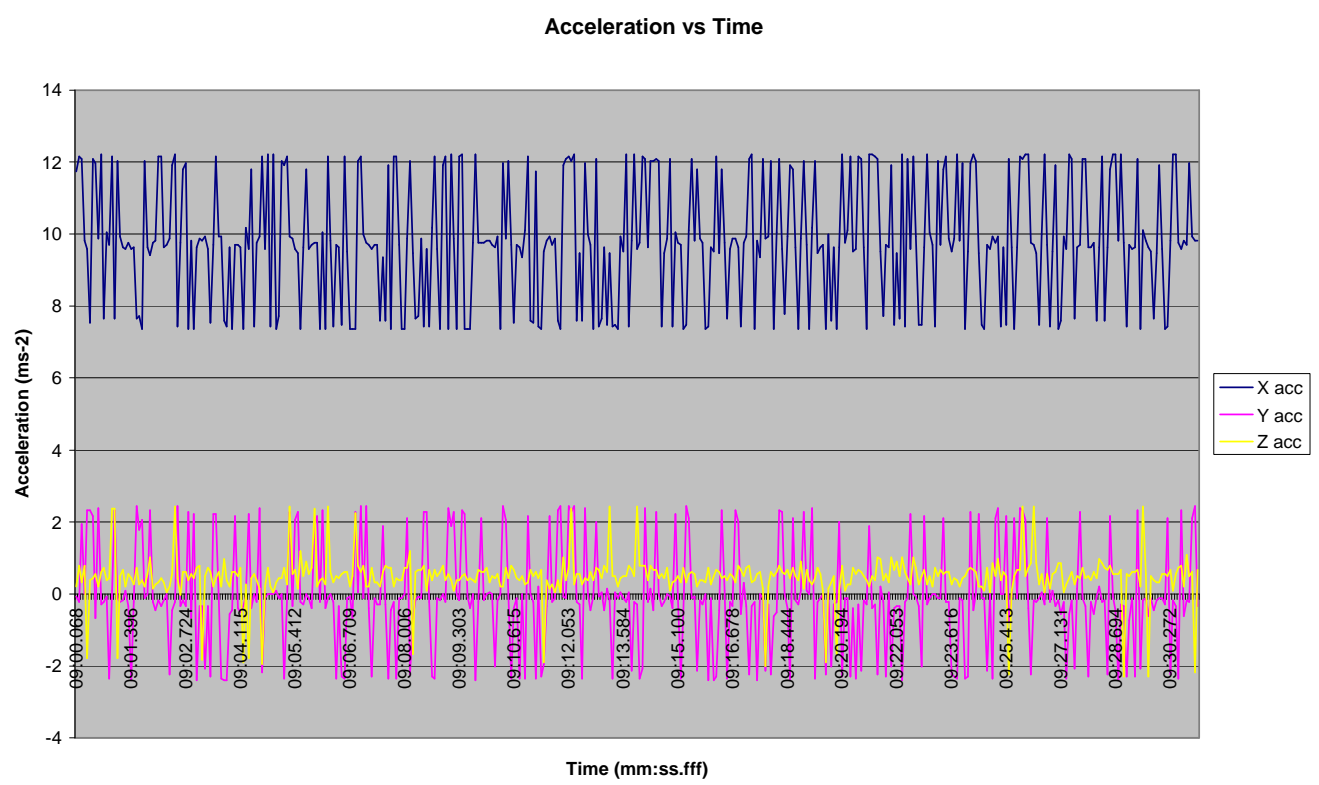

Figure 5.2 Positive X Acceleration Reading from a Static Platform

If the platform is rotated so that the $\mathrm{X}$ direction is now negative, the graph in Figure 5.3 shows the results. The negative $\mathrm{X}$ direction has very little swing over a 30 second compared to the positive $\mathrm{X}$ direction. $\mathrm{Y}$ and $\mathrm{Z}$ also show a much lower acceleration swing in Figure 5.3, than the in Figure 5.2. The median for the $\mathrm{Y}$ and $\mathrm{Z}$ directions is $0.612 \mathrm{~ms}^{-2}$ and $-0.660 \mathrm{~ms}^{-2}$ and the median for negative $\mathrm{X}$ direction is Table 5.3.

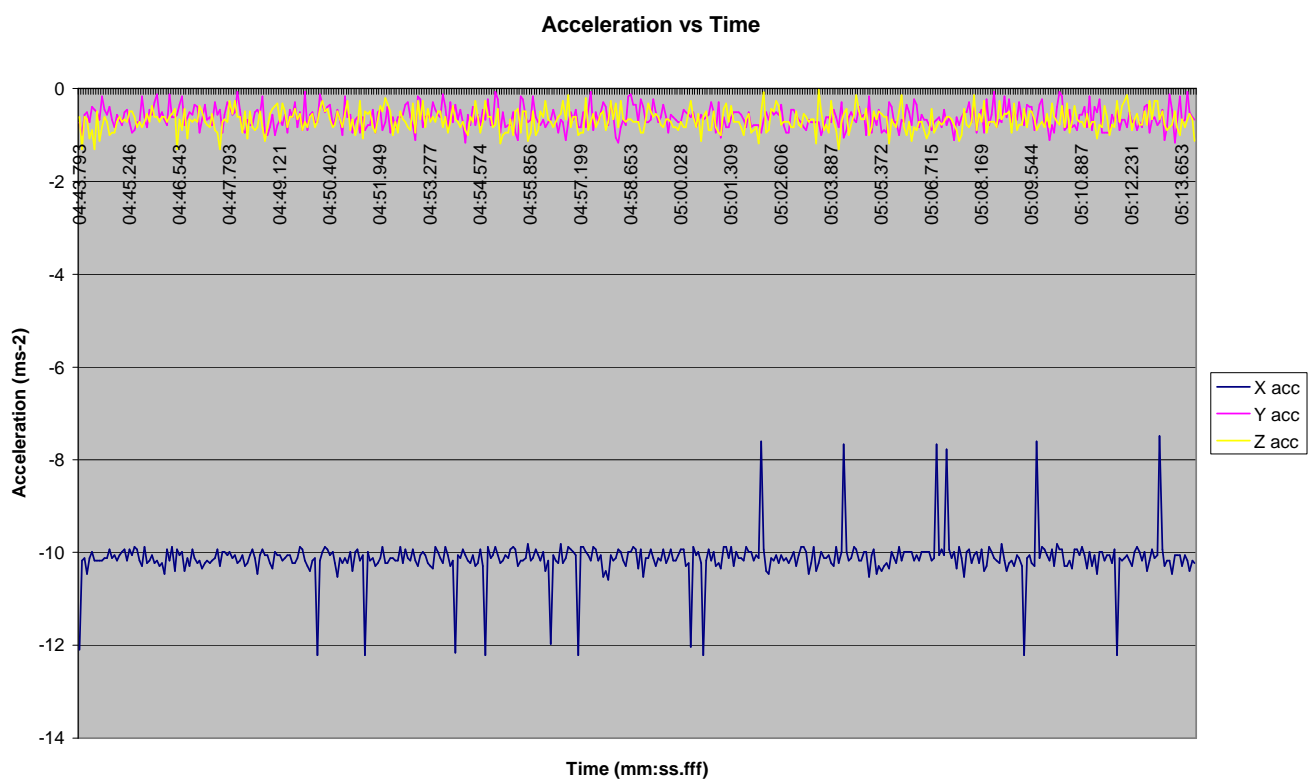

Figure 5.3 Negative X Acceleration Reading from a Static Platform 


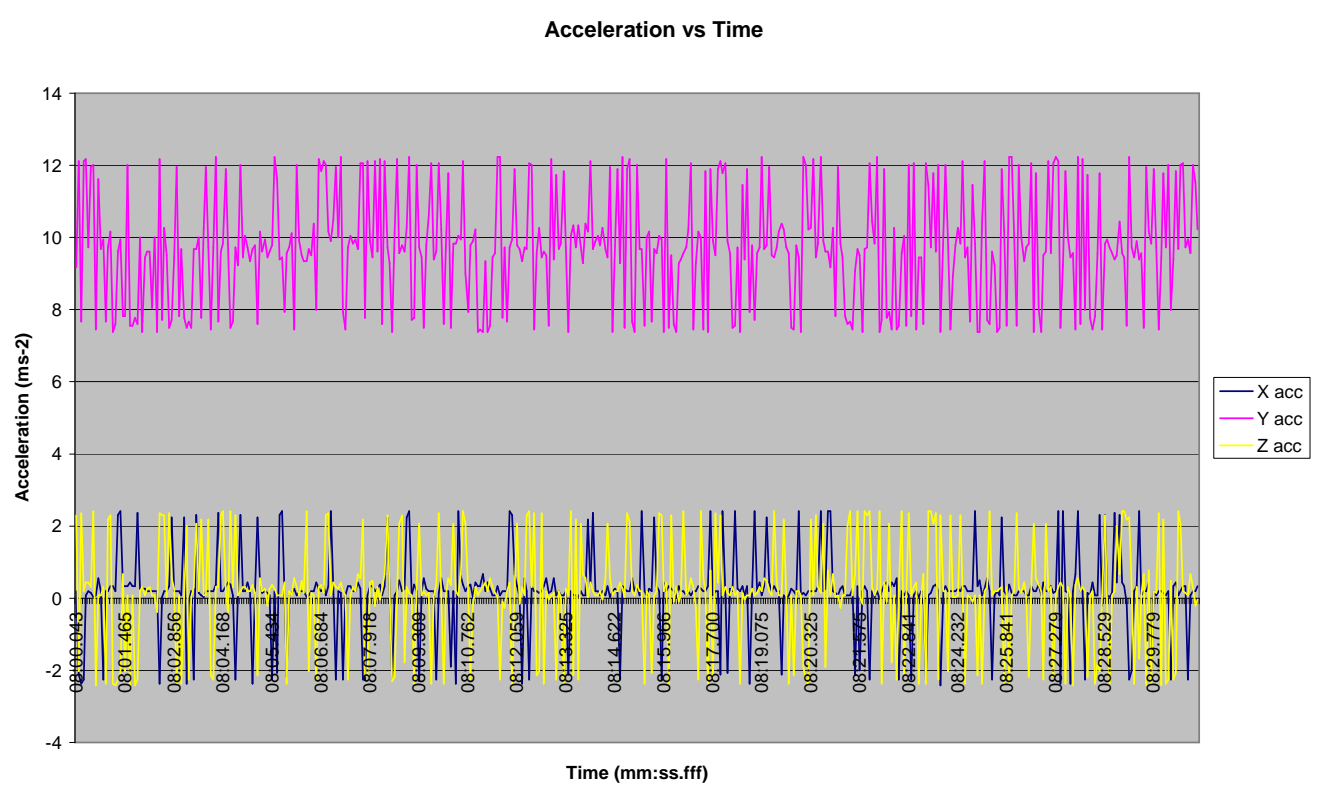

Figure 5.4 Positive Y Acceleration Reading from a Static Platform

Figure 5.4 and Figure 5.5 show the results from the $\mathrm{Y}$ direction. From the graphs, the $\mathrm{Y}$ and $-\mathrm{Y}$ acceleration is what is to be expected based on the orientation of the IMU in the platform. The median values for the directions not under acceleration are within acceptable limits of the accelerometer, being $0.201 \mathrm{~ms}^{-2}$ in Figure 5.4 and $-0.969 \mathrm{~ms}^{-2}$ in Figure 5.5 for the $\mathrm{X}$ direction while for the $\mathrm{Z}$ direction the medians are $0.201 \mathrm{~ms}^{-2}$ in Figure 5.4 and $-1.004 \mathrm{~ms}^{-2}$ in Figure 5.5. 


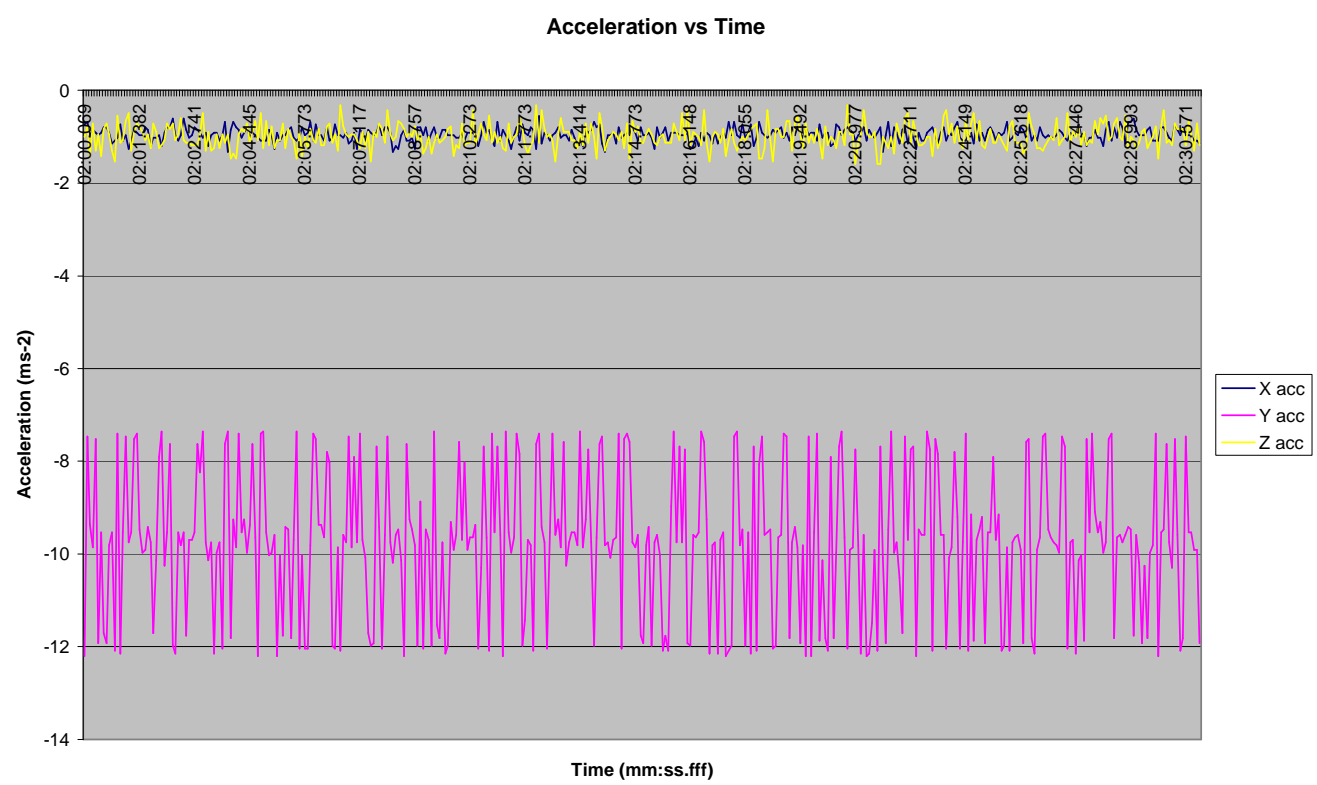

Figure 5.5 Negative Y Acceleration Reading from a Static Platform

Acceleration vs Time

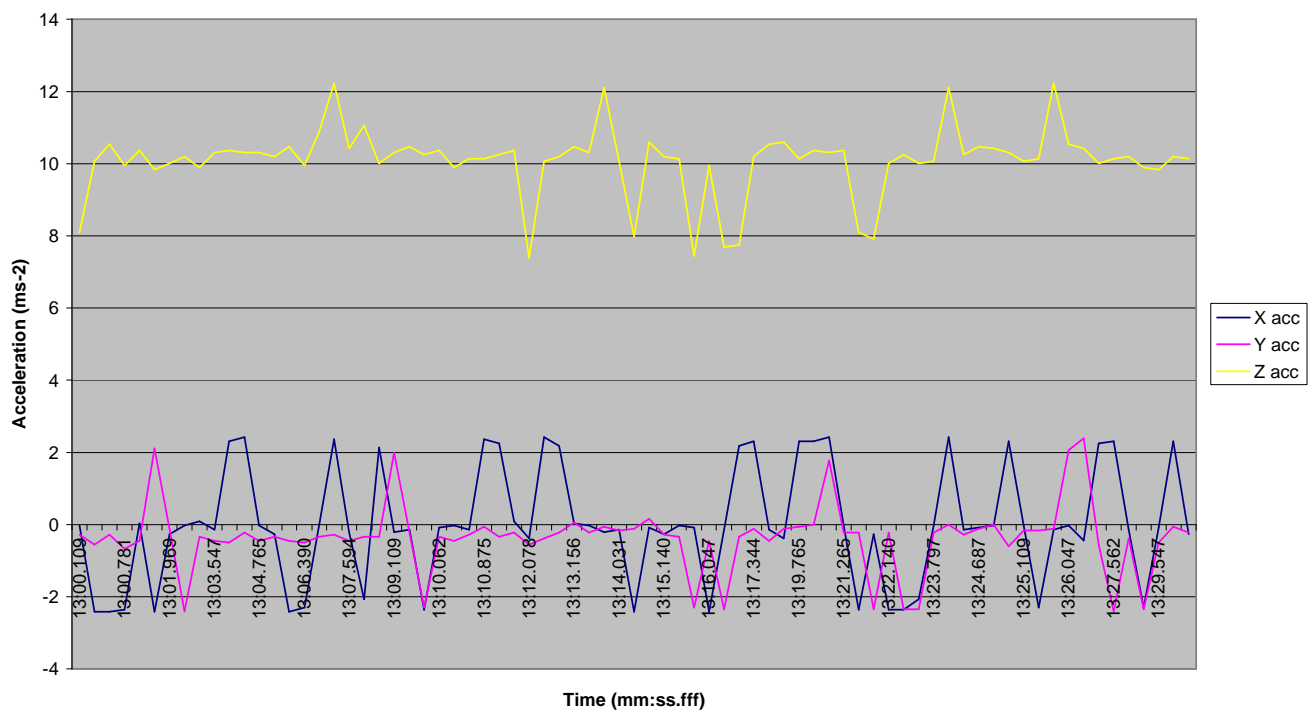

Figure 5.6 Positive Z Acceleration Reading from a Static Platform

Figures 5.6 and 5.7 show the acceleration measured by the accelerometer in the $\mathrm{Z}$ direction in the positive and negative direction. These figures show that the acceleration averages out to $10 \mathrm{~ms}^{-2}$ for the positive direction and $9.8 \mathrm{~ms}^{-2}$ for the negative direction. The graphs also show the $\mathrm{X}$ and $\mathrm{Y}$ acceleration in the respective graphs. Figure 5.7 shows that the measured acceleration is $+1 \mathrm{~ms}^{-2}$ and $-1 \mathrm{~ms}^{-2}$ for the $\mathrm{Y}$ and $\mathrm{X}$ directions. Ideally 
this should be $0 \mathrm{~ms}^{-2}$ because the vice was set up to so that gravity was only working in the $\mathrm{Z}$ direction. This is also shown to a greater extent in Figure 5.6 with an acceleration swing from $-2 \mathrm{~ms}^{-2}$ to $+2 \mathrm{~ms}^{-2}$.

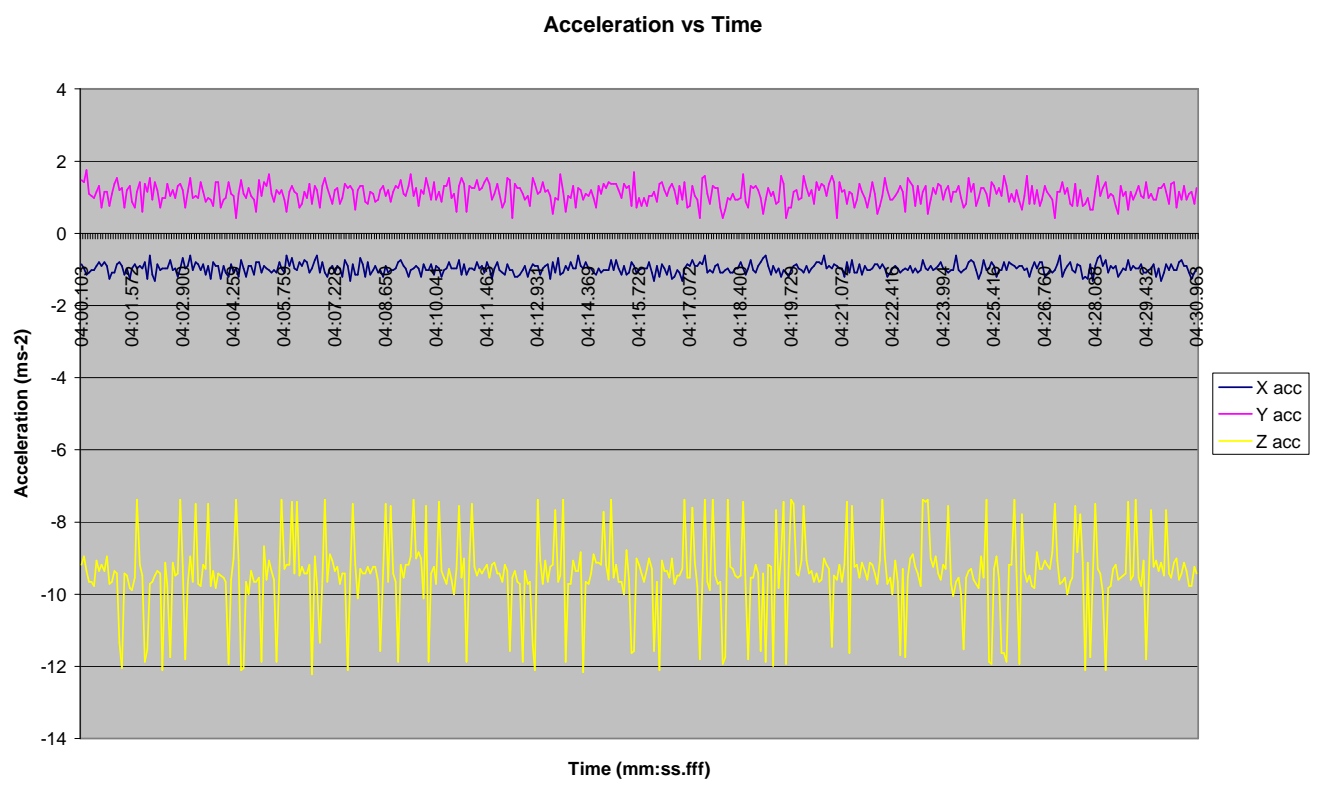

Figure 5.7 Negative Z Acceleration Reading from a Static Platform

The time scale on the $\mathrm{x}$ axis is in the format that has been set up in the Matlab serial_store function as shown in Section 4.1.3. The gyroscope was setup in a similar way to the accelerometer. The board was held in the vice and the vice was placed on a desktop. The microcontroller was turned on and the serial_comms, serial_store and serial_disconnect were used to gather the data that was transmitted to the serial port on the operator's laptop. 


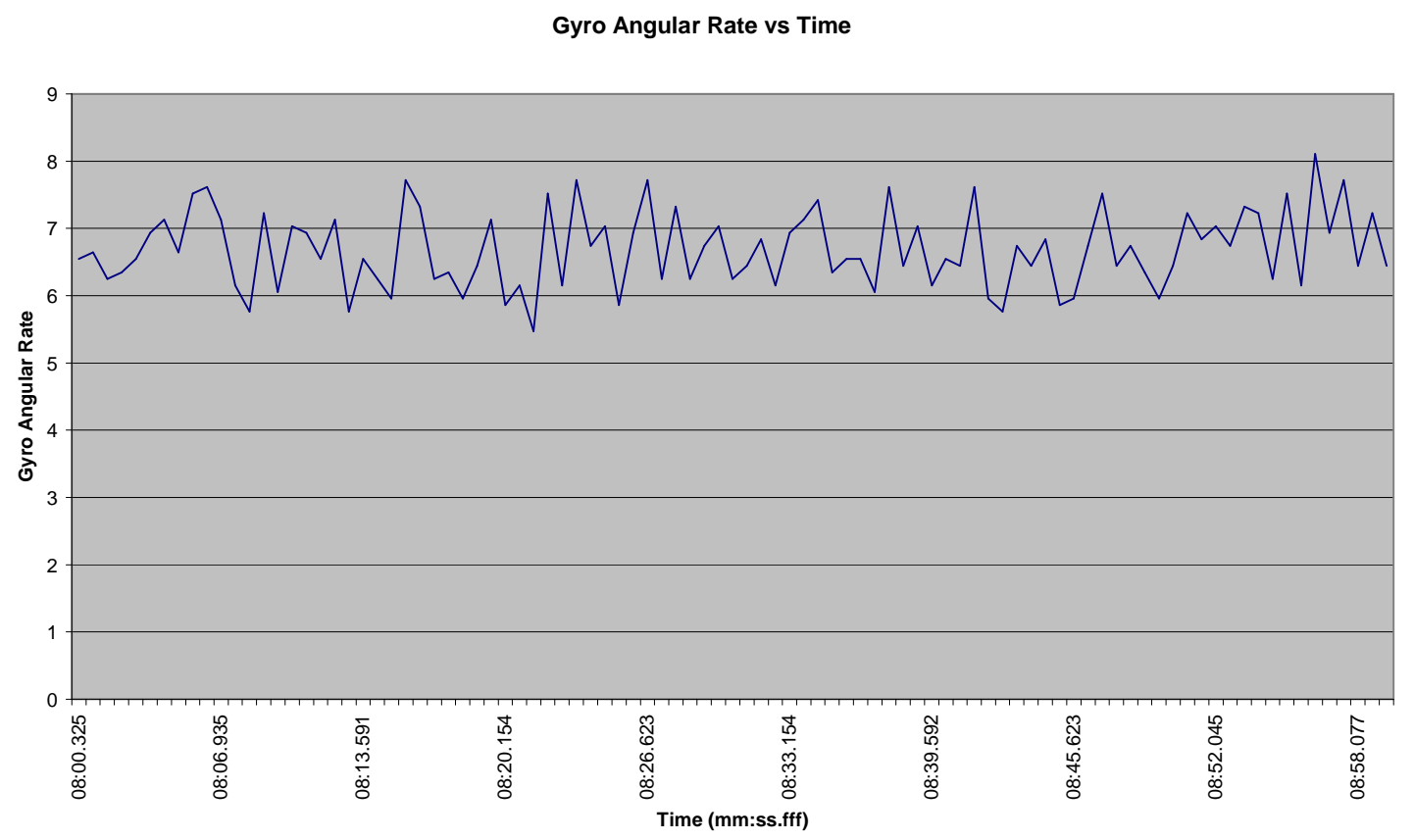

Figure 5.8 Gyroscope Reading from a Static Platform

Figure 5.8 shows the data gathered from the gyroscope over a one minute period. It can be seen that there is a small oscillation between $7.5 \% \mathrm{sec}$ and $6 \% \mathrm{sec}$. The median value for the gyroscope on a stationary non-rotating platform is $6.641 \% \mathrm{sec}$. This infers that the gyroscope is moving when it was not. Possible reasons for this have not been investigated fully but this offshoot could be removed in coding by shifting the zero position negative $6.641 \%$ sec. This would have to be calibrated for each individual gyroscope to ensure that each offset is correctly matched to the respective device. Figure 5.9 shows the range of values and the transmitted bit patterns available from the gyroscope. 


\begin{tabular}{l|l|l}
\hline Angular Rate $\left({ }^{\circ} / \mathrm{sec}\right)$ & Code & Bit Pattern \\
\hline 80 & 2867 & 0000101100110011 \\
$\ldots$ & $\ldots$ & $\ldots$ \\
0.19532 & 2050 & 0000100000000010 \\
0.09766 & 2049 & 0000100000000001 \\
0 & 2048 & 0000100000000000 \\
-0.09766 & 2047 & 0000111111111111 \\
-0.19532 & 2046 & 0000111111111110 \\
$\ldots$ & $\ldots$ & $\ldots$ \\
-80 & 1229 & 0000010011001101 \\
\hline
\end{tabular}

Figure 5.9 Gyroscope Data Coding, Offset Binary

Any data that is received that is outside the range shown in the Figure 5.9 could either be ignored or setup to alert the operator to a serious problem with the vehicle.

In all the experiments there is noise being picked up on the sensors that are not in the plane of the induced acceleration. In some tests this noise was as much as $\pm 2 \mathrm{~ms}^{-2}$ with the induced axis having an acceleration of $10 \mathrm{~ms}^{-2}$. This noise is much larger that the cross axis acceleration pick up of $\pm 3.5 \%$ of the measured acceleration. Possible causes for the noise on the non-induced lines could be vibration of the PCB as the board was anchored at one end dependant on which axis was being tested. It could also be vibrations travelling up from the test platform into the PCB via the stand the PCB was held with. Other causes for the high electrical noise are currently unknown.

\subsection{Analog Devices ADIS 16350}

The Analog Devices ADIS 16350 is a complete IMU comprising of a triple axis accelerometer, a triple axis gyroscope and an internal temperature sensor in a single package. This is a new device, having been designed and manufactured in 2007. An order was placed in June 2007 for a unit. Since it is a new product and, the lead time on this product was 12 weeks, our first unit was received at the start of September 2007. 


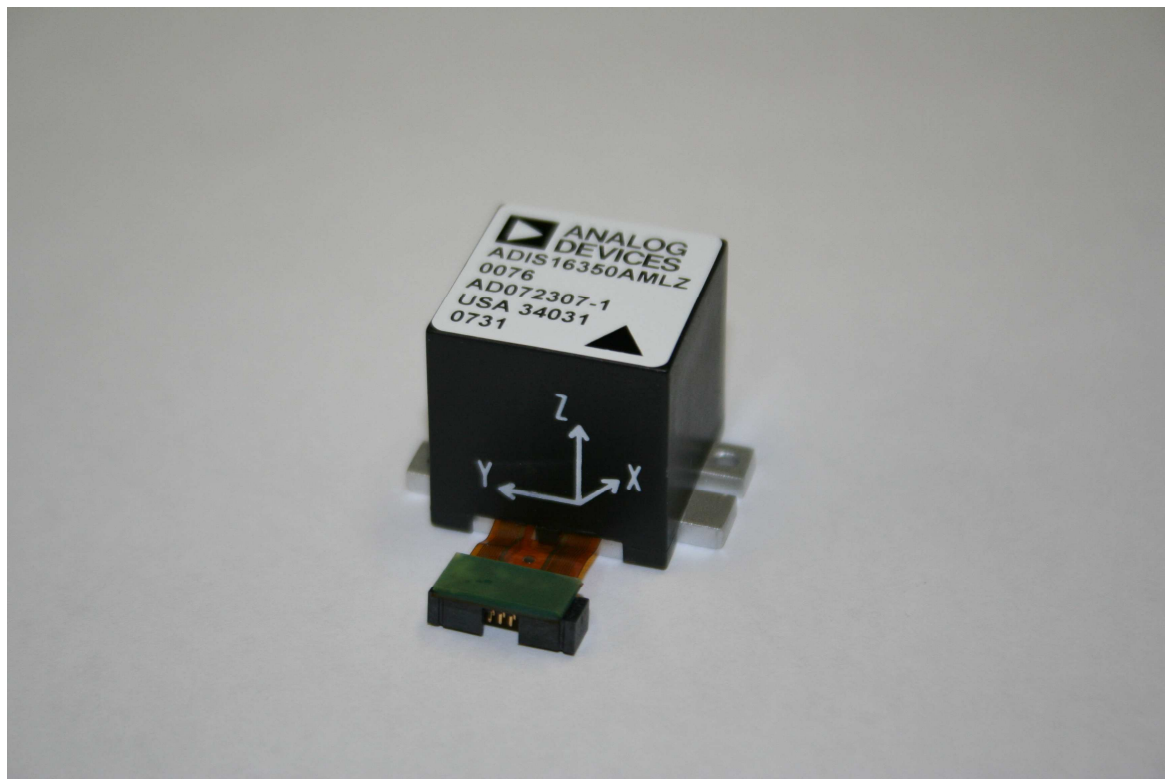

Figure 5.10 Analog Devices ADIS16350 Inertial Navigation Unit

The external dimensions of the package are $23 \mathrm{~mm} \times 23 \mathrm{~mm} \times 23 \mathrm{~mm}(\mathrm{w} \times 1 \times \mathrm{h})$, which makes it the perfect size for the ROV because of the small volume it takes up in the main hull (Section 3.2.1). It is also an SPI interface device, which makes it simple to swap into and interface with the ROV's microcontroller, as in the functional block diagram (Figure 5.11). It has auxiliary general inputs (DIO1 and DIO2) as well as auxiliary Analogue to Digital (AUX_ADC) and Digital to Analogue (AUX_DAC) conversion inputs that allow for extra sensors or signals to be added without extensively rewiring the micro and ROV. 


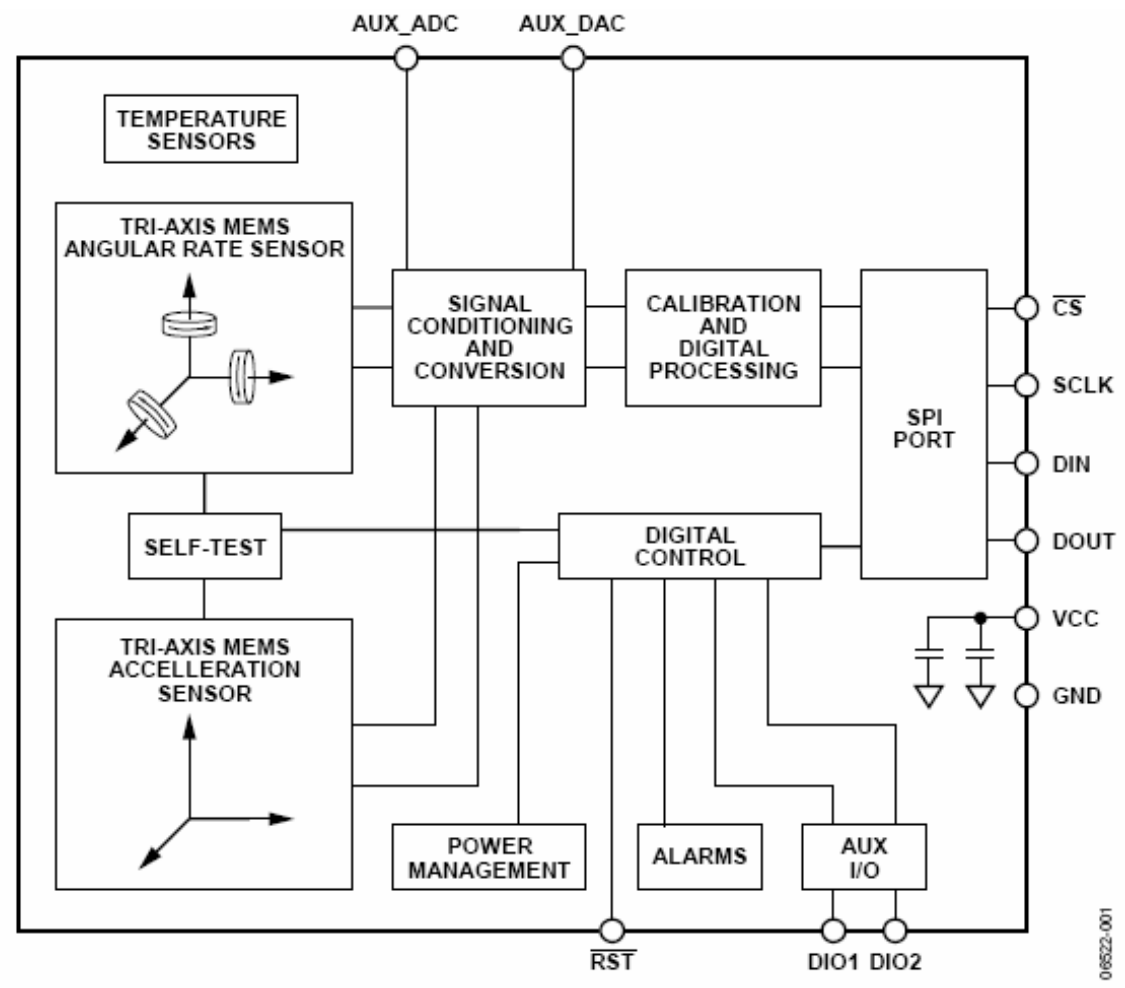

Figure 5.11 Block Diagram of the ADIS16350

The device has a vibration Gyroscope (Section 2.3) with a selectable range from $+/-300^{\circ}$, $+/-150^{\circ}$ and $+/-75^{\circ}$ degrees per second. The Tri axis accelerometer has a range from $+/-$ $10 \mathrm{~g}$ and is based on the differential capacitance principle in Section 2.2. For the ROV's use the gyroscope range will be set to $75^{\circ}$, as this is expected to be the best setting for the ROV's purpose.

Initially the ADIS16350 was going to be compared between the IMU that we are developing and a commercially available one, but because of delays in getting the correct connector to mate with the ADIS16350, it has been unable to be compared to the one that we have built. 


\section{CHAPTER 6. CONCLUSIONS}

\subsection{Conclusion}

The initial ROV upgrade [22] was a effective platform for goal of developing a fully autonomous, fully submersible robotic vehicle. It featured a full micro ATX size computer, complete with $10 \mathrm{~GB}$ hard drive for program storage, and an updated video system that allows the operator to better view the environment 'seen' by the ROV. A new transformer box was created to split the input power of $240 \mathrm{~V}$ into the required voltages for the four motors, the onboard computer, and the various sensors that were kept from the original system.

The software installed consisted of Microsoft Windows 98 Second Edition as the main operating system with LabVIEW from National Instruments as the main control program for the ROV's various systems and sensors. Additional software, such as Philips Webcam, was also used to interface between LabVIEW and the sensors where needed. A remote desktop connection was utilized from the operator's laptop, to allow the operator to run the ROV's programs while underwater.

The chassis was modified [22] to allow individual hulls to be removed for inspection and to facilitate making changes to the hardware inside the hulls. The modification involved the installation of cable conduits that connect the main hulls with the port and starboard hulls. To get into the main hull still entailed removing the cable harness and buoyancy block in order to get to the bolts that held the main hull and the front bulkhead together. Because half a day's work is required for this and the constant damage being done to the aluminium chassis by the steel bolts, Helicoil inserts were added by the author. These allow the front bulkhead to be unbolted from the main hull without the need to remove the cable harness or the buoyancy block. Notches were also milled into the front 
bulkhead so the bulkhead can be levered from the main hull without risking damage to the O-ring inside.

The computer was upgraded to improve the computational power and add greater functionality to the ROV. The placement of internal parts was also changed to add protection to the parts in case water enters the hull. The latest operating system from Microsoft was installed as well as Matlab, which is now handling all the calculations and controls instead of LabVIEW. A new network cable was added to the umbilical, to allow full duplex data transmission from the ROV to the operator's surface based PC.

The microcontroller for the motors was modified so it could interface with the IMU via the SPI interface protocol. This allows other sensors and peripheral devices (such as flash ROM or external analogue to digital conversions) to be added at a later date without the need for a major redesign or change to the hardware. An auto in-system programmer was set up so that the ROV doesn't need to be returned to the surface to reload code into the microcontroller.

An Inertial Measurement Unit was built based on three single-axis gyros and a tri-axis accelerometer to cover all six degrees of freedom of the ROV. The initial testing is set up with just a gyroscope covering the yaw axis and the tri axis accelerometer. Results show that the accelerometer and the gyroscope are functioning as planned. The accelerometer and the gyroscope can not be run at the same time at the moment due to a timing issue involving the polarity of the clock signal. The gyroscope transmits data from the MISO line on the falling edge of the clock signal whereas the accelerometer transmits its data on the rising edge of the clock signal. Attempts to change the clock polarity between getting the data from the accelerometer and the gyroscope have so far been unsuccessful. 


\subsection{Future Work}

There are some areas where major improvements to the ROV and its systems would greatly enhance its ability to carry out the tasks it is designed for.

\subsubsection{Data Optimization and Compression}

The large amount of data to be collected and transmitted to the operator's PC (from the web camera, the IMU and the various other sensors), means that there needs to be some optimization and compression of the data. The data from the IMU and the various other sensors are the easiest ones to optimize because they require less computational power to compress them compared to video images from the camera.

The array used to store the IMU data is the first place to optimize and compress the data. Currently this array is $40000 \times 9$ (rows by columns) which is pre-allocated to set aside the initial memory space - once this is full the array expands as more data points are read in which increases the size and is extremely slow. To optimize this, the array will have to be decreased in size to limit the amount of memory it uses, and then continuously flushed with new data. A possible way to do this is to remove the oldest piece of data from the array, shifting the rest of the data down one position and then writing the new data at the latest position in the array.

The next place to optimize the code would be with the web camera's code: recording video images is another memory intense operation that needs to be optimized. Since the length of time that the ROV operates is only limited by the operator's endurance, the resulting image files from the camera could end up being two or three hours long. This means that the memory will have to be cleared of video image data. Writing to an avi file only stores the images; it does not clear the memory, so this will have to be done as well as writing to the file. 
By doing these operations it is possible to leave enough memory space for Matlab to carry out the other functions without slowing down or stalling Matlab and potentially crashing the control system.

\subsubsection{Kalman Filtering}

Kalman filtering [28] [29] is a method used to reduce the errors inherent in relative position navigation techniques. It compares the previously predicted state to the current measurements and predicts what the current state is. The current state is then used to predict the next state. The same model is also used to update the process error due to noise in the system as shown in Figure 6.1.

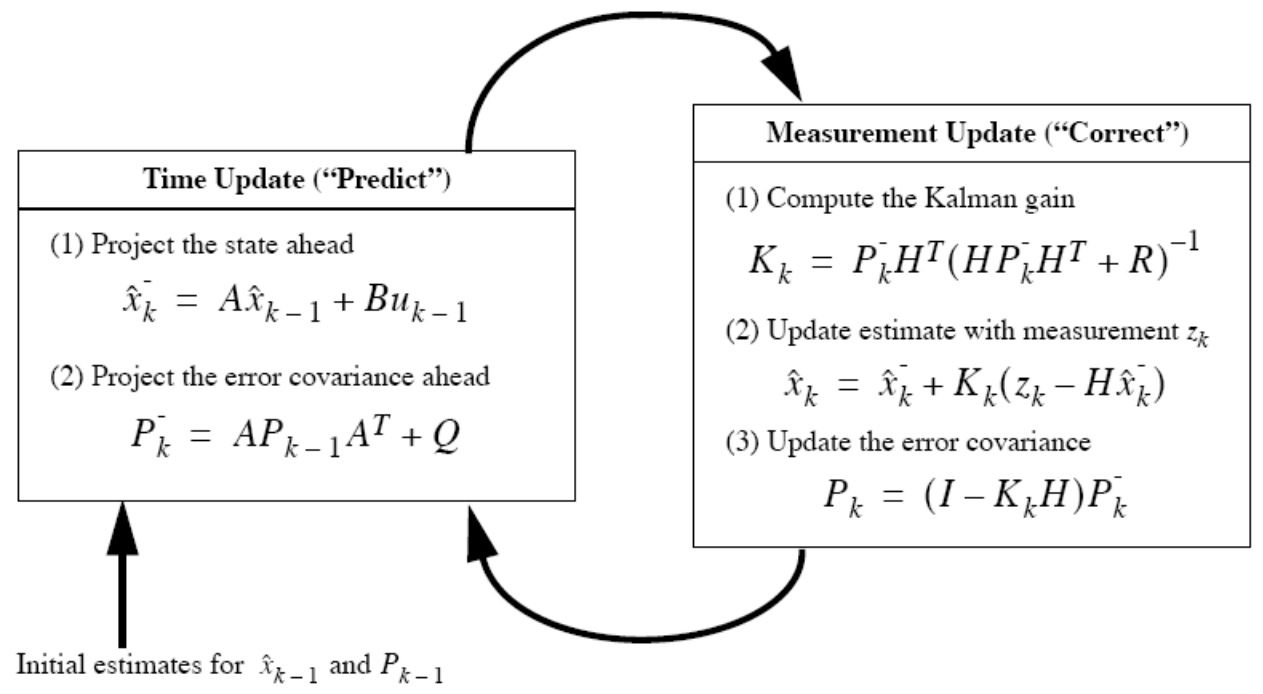

Figure 6.1 Complete Kalman filter Operation

In the predict phase, $A$ is the state transition matrix which maps from the old state to the new state and $Q$ is any noise or interference that produces an uncertainly within the data. For the measurement phase, $H$ is the physical observation matrix of the system or the matrix showing which value was measured, $R$ is the matrix that holds the measured noise in the system. 
This could be implemented in Matlab, and would allow the filtering to be easily tweaked depending on the configuration of the ROV.

\subsubsection{Solid State Hard Drive}

The current hard drive in the ROV is mechanical platter type hard drive. These disks can be damaged by being knocked while the disk heads are moving across the disks. The solution to this is to replace the mechanical drives with solid state hard drives. Solid state hard drives contain no moving parts, so in theory they can never wear out, and are also more robust and suited to mobile solutions. Using solid state hard drives also allows for faster read, write and access times to the data on the disk, while reducing the power used and the heat produced.

The current size of the mechanical hard drive in the ROV is 160 gigabytes, which enables not only the data from all the sensors to be stored but also allows for the video feed to be saved to the hard disk, in addition to all the programs that the ROV requires for operation.

The replacement solid state hard drive must have capacity that is the same as, or greater than, the current mechanical hard drive. However, current sizes are far below the required capacity and prices for these hard drives are currently several hundreds of US dollars for a 32 gigabyte hard drive. When the prices of solid state hard drives are comparable to mechanical hard drives then the current mechanical one should be replaced.

\subsubsection{Wiring}

The wiring in the ROV has accumulates over the various projects that have been done on the ROV. This has lead to there being multiple wires carrying the same signal running throughout the hulls. 


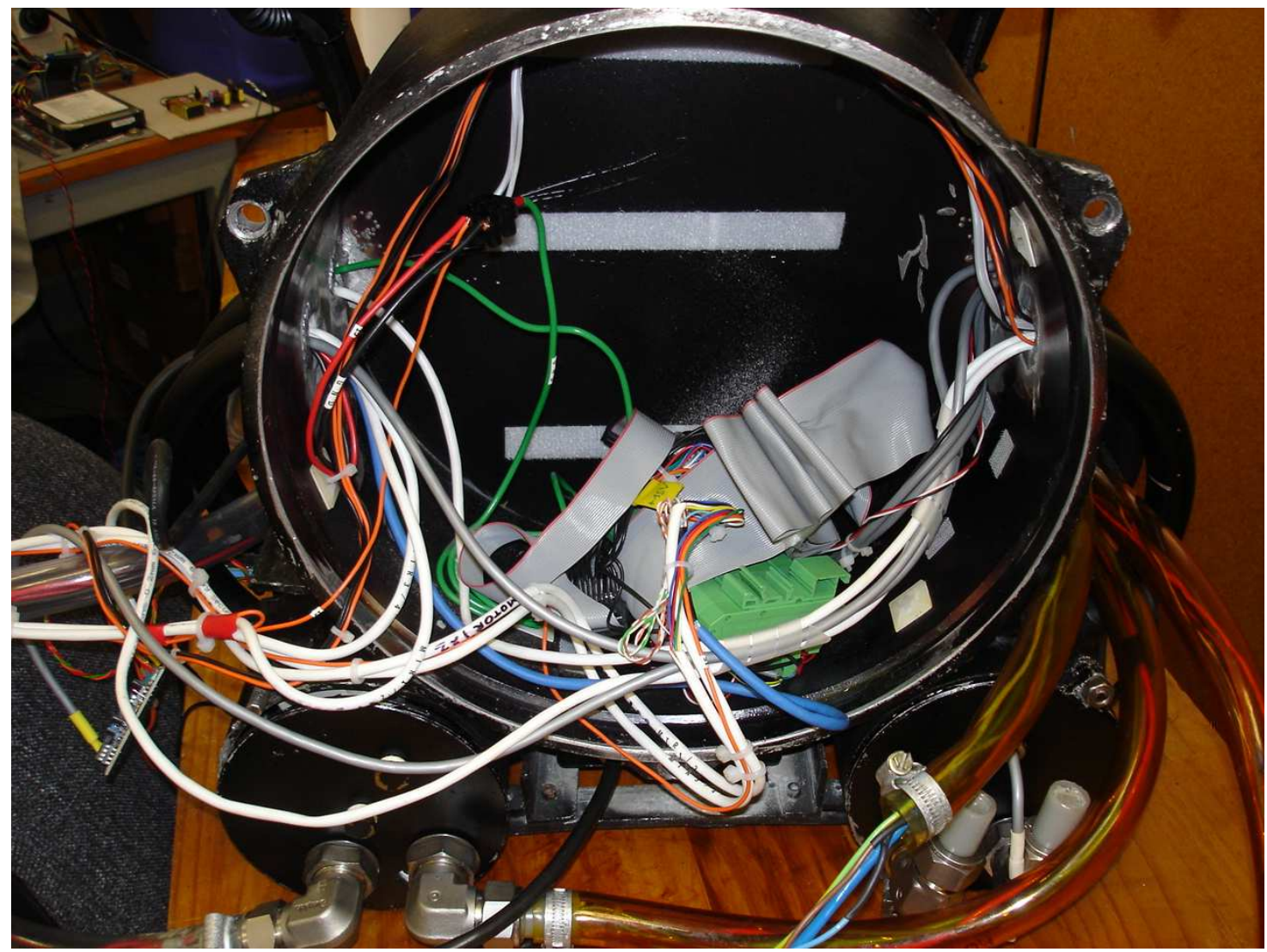

Figure 6.2 State of Wiring in the ROV

A possible solution to this is to convert all the wires in the hulls into a bus system. This bus system would be better for the distribution of power and signals between the three hulls because multiple lines carrying the same signal would be replaced by one line. This would increase the amount of available space in the hulls, as well as making it easier to upgrade and add new parts to the ROV because the lines are already in place.

The bus system would also make it easier to remove and replace components because the units could be plugged into the bus rather than being hard wired (as is the current setup).

\subsubsection{Motors}


The current motors in the ROV are $110 \mathrm{~V}$ industrial drill motors. These are inadequate for controlling the ROV. As stated in Reworking of a Remotely Underwater Vehicle Part II by Gerhard Jungwirth, Markus Mairhofer, Thomas Schmitzberger and Christoph Wagner[27], the motors are prone to damage from the cooling fins, arranged around the centre shaft to blow air all over the coils inside the motor. In one of the motors, these fins have come into contact with the coils on the sides of the motor and scraped the insulation off these coils. This could cause shorting between the coils, damage to the motor and the motor driver circuits, and loss of power to the ROV.

One of the biggest problems with motors such as these is that increasing the umbilical cable that supplies power to the motors increases the losses in that power cable for every metre of length added. This loss $\left(P_{\text {loss }}\right)$ is mainly due to ohmic losses in the cable. The current flowing through cables is given by:

$$
I=P / V
$$

With $P$ being the output power of a system and $V$ being the voltage at which this power is delivered. With $R$ being resistance of the cable the power lost is:

$$
P_{\text {loss }}=R I^{2}=R(P / V)^{2}=R P^{2} / V^{2}
$$

Until batteries or a power plant capable of supplying the required power to drive the motors are created, the power umbilical cable will have to stay.

If the motors were changed to Brushless DC motors some of the troubles with the current motors could be avoided. This includes the damage to the motors, reduced electrical noise and enables the motors to have higher reliability. One of the problems with brushless DC motors is that maximum power settings can create large amounts of heat that could damage the magnets of the dc motors. This would be offset in the ROV though, by the fact that the motors are always emerged in water, which would cool the motors down. 
There is no specific data on the motors them selves apart from the horsepower rating and the supply voltage which is $1 / 2$ horsepower and $110 \mathrm{~V}$ respectfully. A search on the internet turned up some data but the data cant be confirmed as being for these motors. This gives the ROV a top speed of $0.8 \mathrm{~ms}^{-1}$ [22]. The new motors would ideally be $1 / 2$ horsepower (372 Watt) or greater motors. Some possible motors are shown below.

\begin{tabular}{|l|l|l|l|l|}
\hline & $\begin{array}{l}\text { Transmotec } \\
\text { B8698N-48 }\end{array}$ & $\begin{array}{l}\text { Pittman } \\
071215000\end{array}$ & $\begin{array}{l}\text { Submertec } \\
\text { ARS-U1000 }\end{array}$ & $\begin{array}{l}\text { Allied } \\
\text { Motion }\end{array}$ \\
\hline Watt & 633 & 176 & 1000 & $350-580$ \\
\hline VDC & 48 & 74 & $145-160$ & $12-180$ \\
\hline RPM & 3000 & 3400 & 3000 & \\
\hline
\end{tabular}

Table 6.1 Possible Motors for the ROV

\subsubsection{Other work/sensors}

To complement the current array of sensors (which include temperature, humidity, pitch, roll and a compass in the ROV) the following sensors could also be included in the suite. For navigation and localisation:

- A sonar array could be included for close inspection of underwater objects and location of buried objects.

Environmental sensors such as the following could be included depending on the configuration and the task that the ROV was carrying out at the time.

- A salinity sensor to measure the salinity of the water.

- $\mathrm{A} \mathrm{pH}$ sensor to measure the acidity of the water.

Ideally these sensors would be setup in a system that allows them to be changed quickly on the support vessel with very little additional rewiring to be done. This would be best accomplished if the proposed bus wiring structure was installed as stated in Section 6.2.4. 


\subsection{Summary}

The development of a low cost Inertial Navigation System based on tri-axis accelerometers and single-axis gyroscope has been constructed. Preliminary testing has shown it is functioning as expected. Initial problems with both the accelerometer and the gyroscope have been overcome and lead to the design and subsequent revisions of a small IMU board for use in the ROV. Minor timing problems prohibit the use of both the gyroscope and accelerometer at the same time, however due to time constraints this could not be fixed in time.

The operator's Graphical User Interface has been changed to make it more intuitive and allow the operator to avoid being overwhelmed with data. The new layout has opened up areas to install or place further controls as the needs arise in the future.

The interior of the ROV has been upgraded, with a new computer and microcontroller to ensure the vehicle is capable of handling the increased work load from the new sensors without affecting the other operations that already exist. This upgrade also increased the space in the main hull giving more room for future additions to the ROV to enhance its effectiveness.

Mechanical alterations to the chassis have allowed for less down time when repairs are being carried out by increasing the ease of access to the hulls as well as minimise the damage that the hull may endure while being serviced.

The changes made to the ROV have increased the performance of the vehicle and created a stepping stone to further increase the ability of the ROV to accomplish its tasks. The detail on accelerometers, gyroscopes and the communication interfaces used by these devices will provide the Mechatronics Group at Victoria University of Wellington a valuable resource for future work on the mechatrons. 


\section{REFERENCES}

[1] "Talon," Global Security, 2007, http://www.globalsecurity.org/military/systems/ground/images/talon_eodrobotsiraq2.jpg

[2] "Roomba," iRobot Corporation, 2007, http://store.irobot.com/product/index.jsp?productId=2804960

[3] D. A. Carnegie, Loughnane, D.L., and Hurd, S.A, "The Design of a Mobile Autonomous Robot for Indoor Security Applications," Journal of Engineering Manufacture-Proceedings Part B, vol. Vol 218, pp. 533 - 543, May 2004.

[4] D. A. Carnegie, and Cordes, J.C, "The Mechanical Design and Construction of a Mobile Outdoor Multi-Terrain Robot," Journal of Engineering Manufacture Proceedings Part B, vol. Vol 218, pp. pp 1563 - 1575, November 2004.

[5] D. A. Carnegie, Payne, A.D. and Chand, P, "The Design of a Pair of Identical Mobile Robots to Investigate Co-operative Behaviours": (Robert Mayer-Scholz Verlag). 2005

[6] D. A. Williamson, and Carnegie, D.A., "Toward Hierarchical Multi-Robot Search and Rescue: Development of a 'Mother' Agent," Autonomous Robots and Agents: (Springer-Verlag) 2007.

[7] D. A. Carnegie, and King, J.C. , "Converting a Deep-water Access Reconnaissance Television into an Autonomous Underwater Vehicle," Journal of Engineering Manufacture - Proceedings Part B, vol. Vol 218, pp. pp149 - 156, January 2004.

[8] "Side Scan Sonar Image of PB4Y Privateer.", http://www.divetheworld.com/Diving/warbirds/PB4Y/index.htm.

[9] A. D. King, "Inertial Navigation - Forty Years of Evolution," GEC Review, vol. 13, p. 10, 1998, http://www.imarnavigation.de/download/inertial_navigation_introduction.pdf

[10] N. Yazdi, F. Ayazi and K. Najafi, "Micromachined Inertial Sensors", Proceedings of the IEEE, Vol. 86, No. 8, pp1640-1659, August 1998

[11] MEMSIC, "MEMSIC Thermally based Accelerometers," 2006, http://www.memsic.com/memsic/products/technology.html

[12] G.E. Stedman, "Ring-laser tests of fundamental physics and geophysics," Rep. Prog. Phys. 60, pp. pp615-688, January 1997

[13] K.A. Killian, "Pointing Grade Fiber Optic Gyroscope", IEEE AES Systems Magazine, July 1994

[14] "SPI Communication," North Dakota State University, http://www.ece.ndsu.nodak.edu/ glower/ee376/lecture/SPI.pdf

[15] "I2C Communication Layout," Philips Semiconductor, http://www.semiconductors.philips.com/products/interface_control/i2c/?gclid=CL bKi4_qhYYCFRgNGAodyl6zGA, last accessed July 2007

[16] I. Omega Engineering, "Analog Signal Transmission: Noise and Grounding." 
[17] "6DOF IMU with Bluetooth," Sparkfun Electronics, http://www.sparkfun.com/commerce/product_info.php?products_id=754

[18] "IMU400," Crossbow, http://www.xbow.com/Products/productdetails.aspx?sid=209

[19] "LandMark 10 IMU," Gladiator Technologies, http://www.gladiatortechnologies.com/product_LANDMARK10_IMU.htm

[20] "MEMSense MAG3," Omni Instruments, http://www.omniinstruments.com.au/products/product/moredetails/accelrate3dtriaxial.id96.html

[21] A. Kelly, "Modern Inertial and Satellite Navigation Systems," The Robotics Institute, Carnegie Mellon University, Pittsburgh, PA CMU - RI - TR - 94 - 15, May 2 1994, http://www.ri.cmu.edu/pubs/pub_334.html.

[22] J. C. King, "The Development of an AUV," in Physics and Electronic Department. vol. MSc Hamilton: University of Waikato, 2002, p. 147.

[23] H. Oelerich, "Directed Improvements to an Autonomous Underwater Mechatron," Department of Physics and Electronic Engineering, University of Waikato, Hamilton 2002.

[24] C. L. Cawley, "The Enhancement of a Multi-Terrain Mechatron for Autonomous Outdoor Applications," in Physics Department. vol. MSc Hamilton: University of Waikato, 2006, p. 230.

[25] "AN2041 Application Note for LIS3LV02DQ: 3-Axis +/-2g / +/-6g Digital Output Low Voltage Linear Accelerometer," ST Microelectronics October 12th 2005.

[26] A. Kelly, "Essential Kinematics for Autonomous Vehicles," The Robotics Institute, Carnegie Mellon University, Pittsburgh CMU-RI-TR-94-14, May 2 1994, http://www.ri.cmu.edu/pubs/pub_333.html.

[27] M. M. Gerhard Jungwirth, Thomas Schmitzberger, Christoph Wagnar, "Reworking of a Remote Underwater Vehicle Part 2," Department of Physics and Electronic Engineering, University of Waikato, Hamilton, 3 month project 2004.

[28] G. Welch. and. G. Bishop, "An Introduction to the Kalman Filter," Department of Computer Science, University of North Caroling at Chapel Hill July 242006.

[29] A. Kelly, "A 3D State Space Formulation of a Navigation Kalman Filter for Autonomous Vehicles," The Robotics Institute, Carnegie Mellon University, Pittsburgh CMU-RI-TR-94-19, May 2 1994, http://www.ri.cmu.edu/pubs/pub_338.html . 

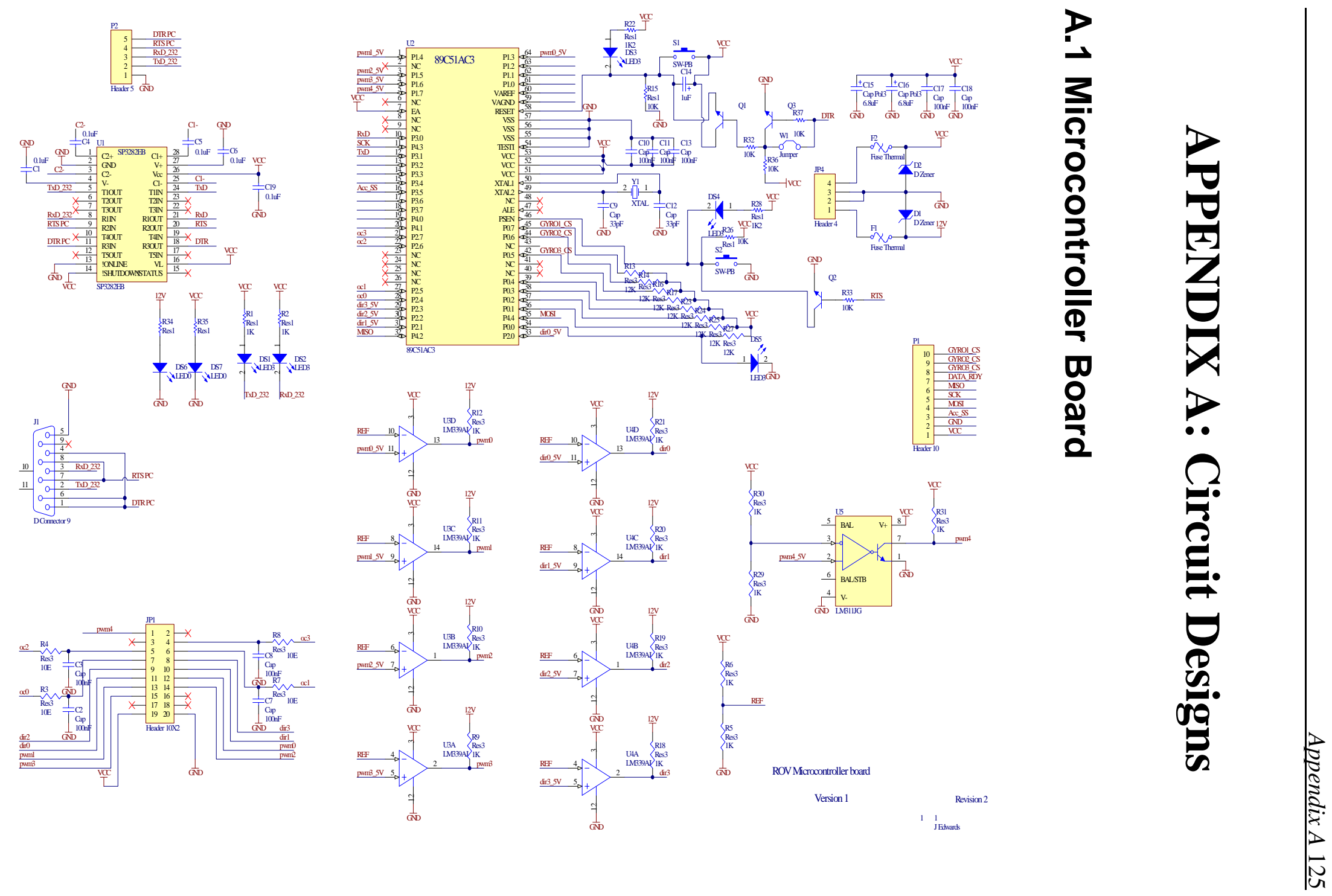

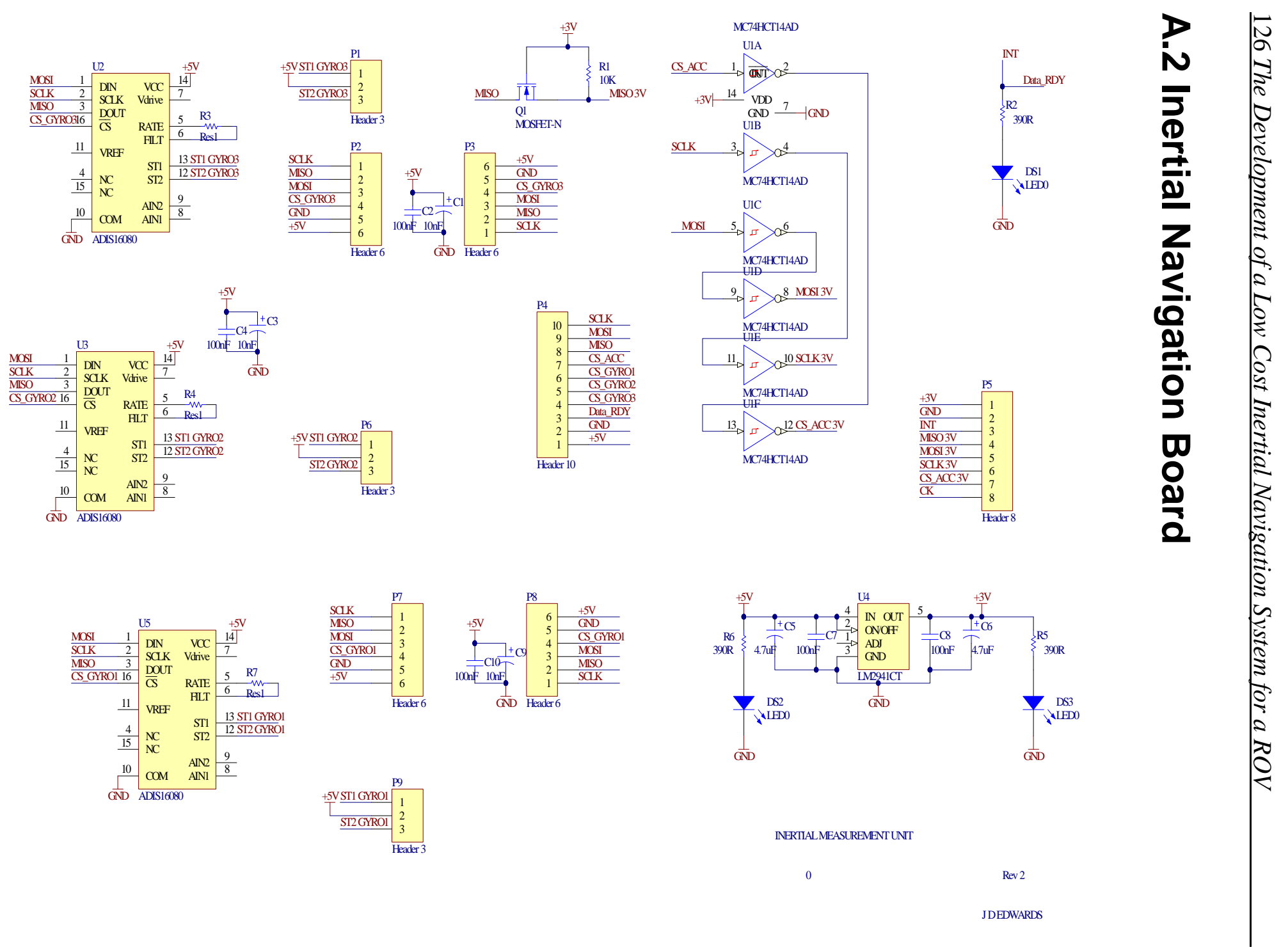

NERTALLMEASUREMENT UNT 


\section{APPENDIX B: CD Contents}

The attached CD contains the following:

Software

- 89C51AC3 Microcontroller C Code

- Matlab Code

\section{Schematics}

- Microcontroller Board

- Accelerometer Board

\section{Datasheets}

- LIS3LV02DQ 3-axis accelerometer

- 89C51AC3 Microcontroller

- ADIS16080 Gyroscope

- LIS3LV02DQ Application Note

- ADIS16350 IMU

\section{ROV Photos}


